\title{
Gráficas Iteradas de Clanes
}

\author{
Miguel Angel Pizaña
}

Tesis Doctoral

Director: Víctor Neumann-Lara

Universidad Autónoma Metropolitana

División de Ciencias Básicas e Ingeniería

Ciudad de México, 23 de Agosto de 2002. 



\section{Miguel Angel Pizaña}

\section{Universidad Autónoma Metropolitana}

Gráficas

Iteradas

de Clanes 



\section{Para Camila}

Sin la cual este trabajo habría sido mucho más fácil, pero no habría valido la pena. 



\section{Agradecimientos}

Quisiera agradecer a mis padres y a mis hermanos por la influencia decisiva que todos ellos han tenido en mi formación como persona, a Violeta, mi amada esposa, por existir y estar siempre a mi lado apoyándome, a mi hija Camila por hacer felices mis días, más allá de lo imaginable.

También quisiera agradecer a los amigos y colegas con los que he tenido la oportunidad de intercambiar ideas sobre el tema de esta tesis en placenteras sesiones de trabajo, en mensajes electrónicos y en charlas ocasionales: Víctor, Paco, Martín, Jayme, João, Célia, Aurora, Marisa, Márcia y Tom. Sin Víctor y Paco, este trabajo simplemente no habría sido posible. También quisiera agradecer a Bruce Hedman por haberme proporcionado tan amablemente copias de sus artículos y a Walter Wallis por su amable atención y por haberme presentado a Tom.

A los profesores que han jugado un insustituible papel en mi formación profesional: Alberto Cervantes, Omar Márquez, Leonardo Salmerón, Martha Rzedowsky, Gabriel Villa, Gerardo Raggi, Francisco Larrión, Víctor Neumann-Lara y tantos otros.

Durante la realización de este trabajo tuve la suerte de contar un extraordinario director de tesis: Víctor Neumann-Lara. Quiero agradecer aquí su inmejorable guía. También quiero agradecer a Francisco Larrión por brindarme generosamente su tiempo y por numerosas observaciones y correcciones. Un agradecimiento especial a ambos por ser tan buenos cuates y por tantas sesiones de trabajo tan placenteras y memorables.

No sería justo no agradecer a los autores de tantos artículos de investigación sobre gráficas de clanes por sentar las bases de este maravilloso tema: Acharya, Alcón, Ariyoshi, Balakrishnan, Bandelt, Bondy, Bornstein, Brigham, Bron, Chen, Chia, Deng, Durán, Dutton, Escalante, França, Frías, Galinier, Gutierrez, Habib, Hamada, 
Hamelink, Hazan, Hedetniemi, Hedman, Howorka, Ide, Kerbosch, Larrión, Lih, Lim, Lin, Lucchesi, Meidanis, de Mello, Moon, Morgana, Moser, Neumann-Lara, Paul, Paulraja, Peng, Peyrat, Prisner, Protti, Rall, Roberts, Sato, Shimogaki, Shirakawa, Simić, Slater, Spencer, Su, Szwarcfiter, Toft, Tsukiyama, Wallis y Wu.

También quiero agradecer a los creadores de la idea de software libre y a los creadores de los programas que he usado para la realización de este trabajo:

Richard Stallman, Linus Trovalds, Leslie Lamport, Donald Knuth, Howard Trickey, Pavel Curtis, Tomas Rokicki, Tim Morgan, Joachim Martillo, Robert Krawitz, Bram Moolenaar, Tim Thompson, Tony Andrews, Fred Walter, Francisco Larrión, Johannes Plass, Tim Theisen, Leonard Soicher, Brendan Mckay, Isabel Araujo, Robert Arthur, Hans Ulrich Besche, Thomas Bischops, Oliver Bonten, Thomas Breuer, Frank Celler, Gene Cooperman, Bettina Eick, Volkmar Felsch, Franz Gähler, Willem de Graaf, Burkhard Höfling, Jens Hollmann, Derek Holt, Erzsébet Horváth, Alexander Hulpke, Ansgar Kaup, Susanne Keitemeier, Steve Linton, Frank Lübeck, Bohdan Majewski, Johannes Meier, Thomas Merkwitz, Wolfgang Merkwitz, Jürgen Mnich, Joachim Neubüser, Max Neunhöffer, Werner Nickel, Alice Niemeyer, Götz Pfeiffer, Udo Polis, Ferenc Rákóczi, Sarah Rees, Edmund Robertson, Ute Schiffer, Martin Schönert, Ákos Seress, Andrew Solomon, Heiko Theißen, Rob Wainwright, Alex Wegner, Chris Wensley, Charles Wright y varios miles más.

Ellos han donado desinteresadamente su trabajo y su conocimiento a la humanidad, haciendo de este mundo un mejor lugar para vivir.

Finalmente, deseo agradecer a las instituciones que me han brindado apoyo en recursos económicos y/o materiales: Conacyt, SNI, UAM, IMATE, División de CBI (UAM), Depto. de Ingeniería Eléctrica (UAM) y al Área de Computación y Sistemas (UAM). 


\section{Contenido}

$\begin{array}{ll}\text { Îndice de figuras } & \text { ix }\end{array}$

Índice de símbolos $\quad x i$

Dependencias lógicas entre capítulos xiii

1 Introducción $\quad 1$

Gráficas de clanes ..................... . . 1

Notación y terminología ................ 3

Resultados preliminares .............. 6

Gráficas clan convergentes ............ . 7

Gráficas clan divergentes . . . . . . . . . . . . 10

2 Cimientos $\quad 13$

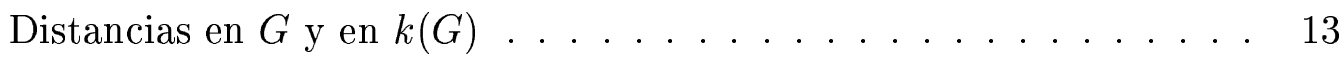

La fórmula de la distancia . . . . . . . . . . . . 18

Gráficas de Johnson . . . . . . . . . . . . . . . . 24

Diámetros ......................... 29

3 Relojes 33

Gráficas cíclicamente segmentadas . . . . . . . . . . . . . 34

Los relojes y sus clanes . . . . . . . . . . . . . . . 34

El $k$-carácter de los relojes . . . . . . . . . . . . . . . . 43

Sumas segmentadas . . . . . . . . . . . . . . . . 46

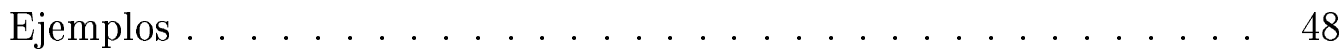

4 Gráficas coafines $\quad 53$

Las categorías $\mathcal{G}, \mathcal{G}^{a}, \mathcal{G}^{(r)} \ldots \ldots \ldots \ldots$ 
El operador de clanes en $\mathcal{G}, \mathcal{G}^{a}, \mathcal{G}^{(r)} \ldots \ldots \ldots \ldots$

Dominación en $\mathcal{G}, \mathcal{G}^{a}, \mathcal{G}^{(r)} \ldots \ldots \ldots \ldots \ldots$

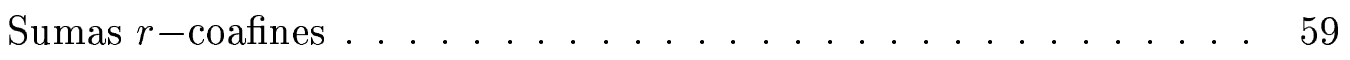

Gráficas divergentes en sumandos . . . . . . . . . . . . 6 60

El teorema de la divergencia en sumandos . . . . . . . . . . . 61

Relojes divergentes en sumandos . . . . . . . . . . . . . . . 62

El icosaedro . . . . . . . . . . . . . . . . 65

Superficies $k$-divergentes $\ldots \ldots \ldots \ldots \ldots 6$

5 Cuello local grande $\quad 71$

Cuello local . . . . . . . . . . . . . . . . . . . . 71

El teorema de triángulos y vértices $\ldots \ldots \ldots \ldots . \ldots 72$

El teorema del cuello local grande . . . . . . . . . . . 75

Superficies $k$-convergentes . . . . . . . . . . . . . . . 78

Triangulaciones del disco . . . . . . . . . . . . . . 84

$\begin{array}{ll}\text { Conclusiones } & 87\end{array}$

$\begin{array}{ll}\text { Problemas abiertos } & 89\end{array}$

$\begin{array}{ll}\text { Bibliografía } & 91\end{array}$

$\begin{array}{ll}\text { Índice } & 99\end{array}$ 


\section{Índice de Figuras}

1.1 Una estrella y una corbata . . . . . . . . . . . 9

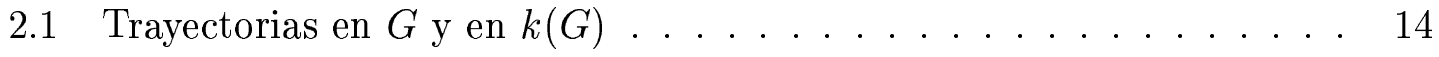

2.2 Cimientos . . . . . . . . . . . . . . . . . . . . . . 19

2.3 Distancias en $G$ y $k^{n}(G) \ldots \ldots \ldots \ldots \ldots \ldots \ldots$

2.4 La construcción de $H(G, d) \ldots \ldots \ldots \ldots \ldots \ldots \ldots$

3.1 Una gráfica de $k$-carácter desconocido . . . . . . . . . 46

3.2 Una gráfica $k$-invariante que no es clan-Helly . . . . . . . . . . 48

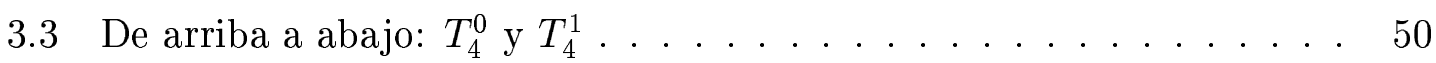

3.4 De arriba a abajo: $H_{2}^{0}, H_{2}^{1}$ y $H_{2}^{2} \ldots \ldots \ldots \ldots \ldots \ldots \ldots \ldots \ldots$

4.1 Icosaedro con una inmersión de $\mathbb{H}_{2}^{0}$ en $k(\mathbb{I}) \ldots \ldots \ldots \ldots$

4.2 Dodecaedro estelado $\mathbb{S}$ : triangulación 5 -coafín de una esfera $\ldots \ldots 66$

4.3 Dodecaedro estelado con una inmersión de $\mathbb{H}_{4}^{0}$ en $k(\mathbb{S}) \ldots \ldots .67$

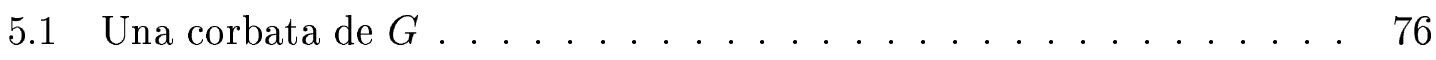

5.2 Otra corbata de $G$ con centro en $T \ldots \ldots \ldots \ldots \ldots$

5.3 Una región fundamental en $\mathbb{Z} \oplus \mathbb{Z}$ que produce a la gráfica $T_{r} \ldots \ldots 80$

5.4 Una triangulación del cilindro localmente $\mathcal{P}_{4} \ldots \ldots \ldots \ldots$

5.5 Todas las gráficas localmente $\left\{\mathcal{C}_{4}, \mathcal{C}_{5}\right\} \ldots \ldots \ldots \ldots$ 


\section{Índice de símbolos}

\begin{tabular}{|c|c|c|}
\hline$A^{+}, 35$ & $F A, 34$ & $N_{G}[A], 4$ \\
\hline$A \cdot B, 5$ & $F_{x}, 34$ & $N_{G}(x), 3$ \\
\hline$A \boxplus B, 46$ & & $N_{G}[x], 4$ \\
\hline$A \otimes B, 4$ & $G^{+}, 40$ & \\
\hline $\mathbb{A} \otimes \mathbb{B}, 54$ & $\bar{G}, 40$ & $\mathcal{O}_{n}, 5$ \\
\hline $\mathbb{A} \succsim \mathbb{B}, 56$ & $G[X], 5$ & $p(G), 2$ \\
\hline $\mathbb{A}=\bigoplus_{\mathbb{G}} \mathbb{A}_{i}, 59$ & $G_{i}^{+}, 40$ & $\mathcal{P}_{n}, 5$ \\
\hline $\mathbb{A}, \mathbb{B}, \mathbb{C}, \ldots, 53$ & $\bar{G}_{i}, 40$ & \\
\hline & $\tilde{G}_{i}, 36$ & $\operatorname{radio}(G), 4$ \\
\hline$b(G, n), 31$ & $\mathcal{G}, 4$ & $\operatorname{radio}_{x}(G), 4$ \\
\hline$B A, 34$ & $\mathcal{G}^{a}, 54$ & $\operatorname{rango}(\mathbb{G}), 59$ \\
\hline$B_{x}, 34$ & $\mathcal{G}^{(r)}, 54$ & \\
\hline $\mathcal{B}_{G}(Q), 18$ & & $\bar{S} 40$ \\
\hline $\mathcal{B}_{k^{m}(G)}(Q), 18$ & $\mathcal{H}, 56$ & $\underset{\widetilde{S}, 40}{\mathcal{S}}$ \\
\hline $\mathcal{B}_{m}(Q), 18$ & $H(G, d), 24$ & $\mathcal{S}, 36$ \\
\hline $\mathcal{B}(Q), 18$ & $H_{m}^{n}, 50$ & $\mathbb{S}, 66$ \\
\hline & $\mathbb{H}_{m}^{n}, 64$ & $\mathbb{T}, 67$ \\
\hline$c(G), 3$ & $i(G), 2$ & $T_{r}^{\prime}, 79$ \\
\hline $\operatorname{cl}(G), 71$ & II. 65 & $\mathbb{T}_{2 m}^{n}, 63$ \\
\hline $\operatorname{cl}(G, x), 71$ & $\mathbb{1}, 65$ & $T^{(r)}, 81$ \\
\hline $\mathcal{C}_{n}, 5$ & $J(M, r), 25$ & $T_{r}, 79$ \\
\hline cuello $(G), 71$ & $J(m, r), 25$ & $T_{s}^{n}, 49$ \\
\hline $\mathcal{D}(A, B), 4$ & $K_{n}, 5$ & $*(x), 8$ \\
\hline $\mathcal{D}_{G}(A, B), 4$ & $k(G), 1$ & $x^{*}, 9$ \\
\hline$d_{G}(x, y), 4$ & $k^{n}(G), 2$ & $x^{+}, 35$ \\
\hline $\operatorname{diam}(G), 4$ & & $\bar{x}, 37$ \\
\hline$d(x, y), 4$ & $L^{i}(G), 25$ & \\
\hline
\end{tabular}




\section{Dependencias lógicas entre capítulos}

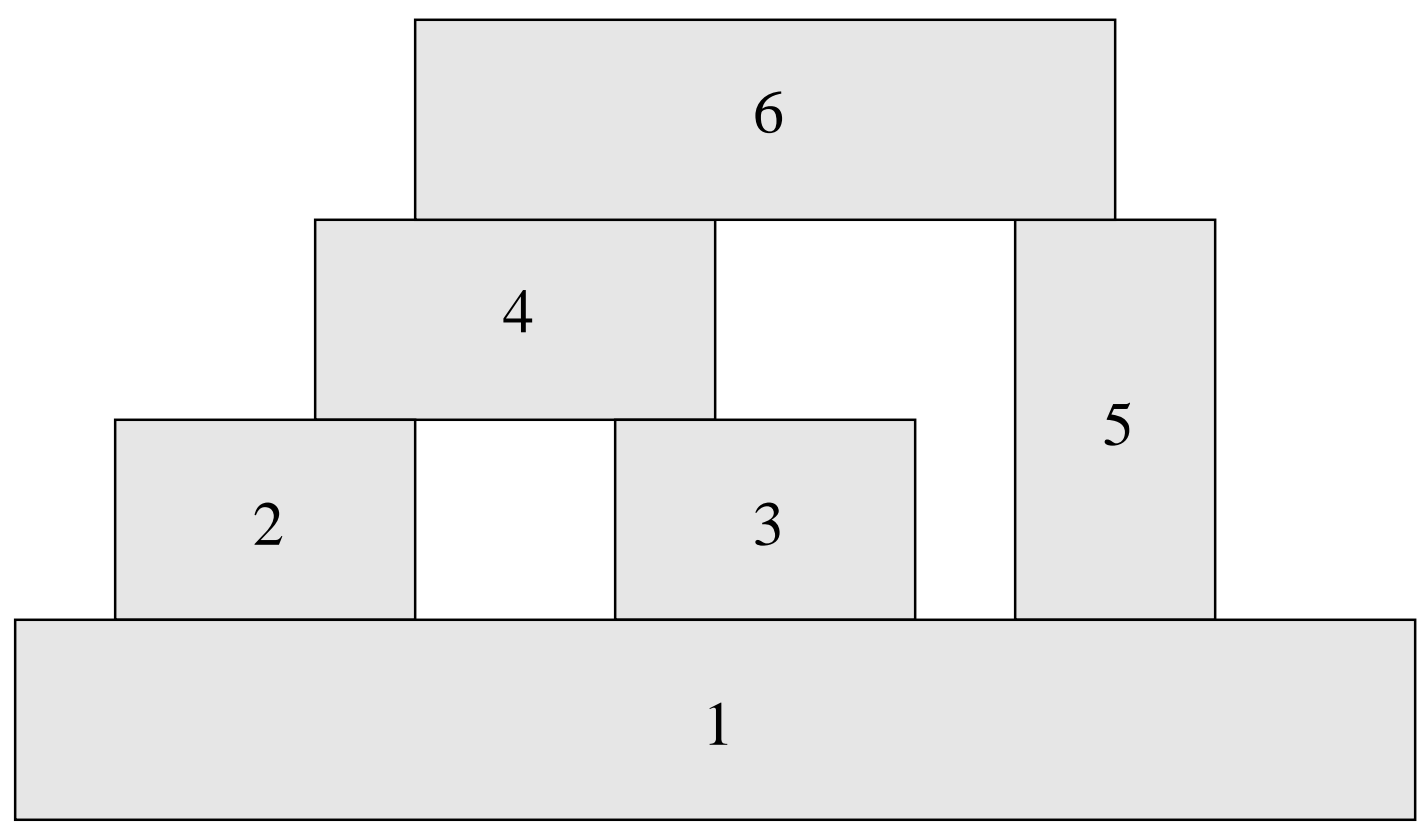

1. Introducción

2. Cimientos

3. Relojes

4. Gráficas coafines

5. Cuello local grande

6. Conclusiones 


\section{Capítulo 1}

\section{Introducción}

\section{Gráficas de clanes}

Si $G$ es una gráfica, un clan de $G$ es una subgráfica completa maximal. Los clanes de una gráfica han sido objeto de estudio prácticamente desde el mismo inicio de la teoría de las gráficas y desde entonces han sido estudiados en una amplia variedad de situaciones. En particular, se sabe que el problema calcular el clan máximo es $\mathcal{N} \mathcal{P}$-duro (el problema de decisión asociado es $\mathcal{N} \mathcal{P}$-completo) desde el mismísimo trabajo seminal de Cook [12](1971) en el que se definen estos conceptos (ver también [20]). De modo que la noción de clan de una gráfica es una pieza fundamental en la teoría de complejidad computacional y su profunda comprensión es esencial para el avance de la teoría.

Incluso con más razón se puede afirmar que la noción de clan es una idea central en la teoría de las gráficas. Una construcción que exhibe la estructura clánica de una gráfica $G$ es la gráfica de clanes de $G, k(G)$, que se define como la gráfica de intersección del conjunto de clanes de $G$. Esto es, $k(G)$ tiene por vértices a los clanes de $G$ y dos vértices son adyacentes si son distintos tienen intersección no vacía. Esta construcción se ha estudiado extensamente al menos desde 1968 (véase [27]).

El operador de clanes $k$, transforma pues gráficas en gráficas y es entonces natural preguntarse qué sucede al aplicar repetidas veces el operador de clanes a una gráfica específica. Las gráficas iteradas de clanes de $G$ fueron introducidas por Hedetniemi y Slater en [31](1972), y se definen recursivamente por medio de las fórmulas: $k^{0}(G)=$ 
$G$ y $k^{n+1}(G)=k\left(k^{n}(G)\right)$. Las gráficas iteradas de clanes han sido estudiadas en múltiples artículos. Este trabajo incluye bibliografía extensa sobre el tema y referencias adicionales pueden ser encontradas en [74] y [66].

Los gráficas iteradas de clanes pueden exhibir una variedad de comportamientos:

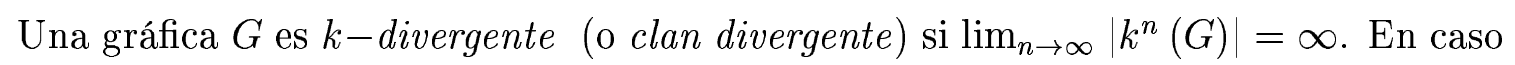
contrario decimos que es $k$-convergente (o clan convergente). Es fácil probar que $G$ es $k$-divergente si y sólo si el conjunto $\left\{\left|V\left(k^{n}(G)\right)\right|: n \in \mathbb{N}\right\}$ no está acotado. También es fácil ver que una gráfica $G$ es $k$-convergente, si y sólo si, existen enteros $i \geq 0$ y $p \geq 1$ tal que $k^{i}(G) \cong k^{i+p}(G)$. Si $i_{0}$ y $p_{0}$ son los número más pequeños que satisfacen la condición anterior decimos que $i_{0}$ es el índice de transición de $G$ y que $p_{0}$ es el periodo de $G$, denotaremos a estos números por $i(G)$ y $p(G)$ respectivamente. Decimos que $G$ es $k$-periódica si $i(G)=0$. $G$ es $k^{n}$-invariante si $k^{n} G \cong G$. En el caso especial en que $k^{n}(G)$ es isomorfa a la gráfica trivial (de un punto) para alguna $n$, decimos que $G$ es $k$-nula. El $k$-carácter de $G$ es la propiedad de $G$ que puede tomar uno de tres valores: $k$-divergente, $k$-convergente pero no $k$-nula y $k$-nula.

Los mismos Hedetniemi y Slater en 1972 [31], exhiben ejemplos no triviales de gráficas $k$-nulas, $k$-periódicas y $k$-convergentes (de periodo 1 y 2 ).

Sólo un año después Neumann-Lara descubre los primeros ejemplos de gráficas clan divergentes: Para $n \geq 3$ todos los octaedros $n$-dimensionales $\mathcal{O}_{n}$ son $k$-divergentes. De hecho $k\left(\mathcal{O}_{n}\right) \cong \mathcal{O}_{2^{n-1}}$. Escalante reporta en [15](1973) este descubrimiento de Neumann-Lara y muestra además que existen gráficas de cualquier posible periodo $p(G) \geq 1$.

El estudio del $k$-carácter había empezado. Desde entonces se han abordado las gráficas iteradas de clanes desde diversos enfoques. Por ejemplo, se ha estudiado la $k$-divergencia en $[15-17,37-41,43,46,55-58,61]$. La $k$-convergencia y $k$-nulidad ha sido estudiada en $[1,5,8,16-18,30,31,33,37,42,45,64]$. También existen trabajos que estudian específicamente la $k$-invariancia [3,5,11,47-49]. Otro aspecto que ha sido muy estudiado es el diámetro de gráficas iteradas de clanes $[3,9,10,14,32,33,35,39,59$, $60,62]$. Prisner es el principal estudioso de la relación entre la homología de complejos simpliciales y las gráficas iteradas de clanes [4,44,63,64]. Otros interesantes trabajos en relación a gráficas iteradas de clanes incluyen $[2,7,13,21-27,50,51,65,67-71,75,78]$.

El presente trabajo ha dado lugar a varios artículos de investigación [16,37, 42-49, 
60-62] e incluye muchos de los resultados ahí publicados y algunos otros.

Así, nuestras aportaciones al estudio del diámetro de las gráficas iteradas de clanes $[60,62]$ se encuentran, junto con nuevos teoremas, en el capítulo 2. El estudio que hemos hecho del $k$-carácter de los relojes $[43,46]$ se encuentra en forma ampliada en el capítulo 3. El capítulo 4 contiene una generalización de la maravillosa teoría de gráficas expansivas de Neumann-Lara [55] que incluye una generalización parcial previamente publicada por mi en [61]. Quizá es ésta la aportación más profunda de este trabajo. Finalmente en el capítulo 5 se presentan los resultados que hemos obtenido en el estudio de las gráficas con cuello local grande [45] y algunas de sus consecuencias. Por razones de tiempo, los trabajos publicados en [16,37,42,44,47-49] ya no encontraron lugar en esta tesis.

A lo largo de este trabajo, se presentan problemas abiertos en su lugar natural y luego se recolectan y presentan de nuevo en el apéndice (pág. 89). Sirva esta lista para estimular la imaginación de aquellas personas que lean esta tesis.

\section{Notación y terminología}

Todas nuestras gráficas son no vacías, simples (sin lazos ni aristas paralelas) y a menos que se diga lo contrario, también son finitas.

En general usaremos notación estándar de teoría de gráficas [6, 28]. Hacemos, sin embargo, algunas precisiones:

Sea $G$ una gráfica. Denotamos al conjunto de vértices por $V(G)$ y al conjunto de aristas por $E(G)$. A veces escribiremos $x \in G$ en lugar de $x \in V(G)$. El orden de $G$ es $|G|=|V(G)|$.

Frecuentemente, identificaremos a las subgráficas inducidas de $G$ con sus conjuntos de vértices. En particular, usualmente, veremos a los clanes como subconjuntos de $V(G)$. Diremos que $Q$ es un clan iterado de $G$ si $Q \in V\left(k^{n}(G)\right)$ para alguna $n \geq 1$.

Ocasionalmente, hablaremos de la gráfica de completas de $G, c(G)$, que se define como la gráfica de intersección de todas las completas de $G$. Observe que $k(G)$ es una subgráfica inducida de $c(G)$.

La vecindad de un vértice $x$ en $G, N_{G}(x)$, es el conjunto de todos los vecinos de 
$x$, es decir $N_{G}(x)=\{y \in G:\{x, y\} \in E(G)\}$. La vecindad cerrada de un vértice $x$ en $G$ es $N_{G}[x]=N_{G}(x) \cup\{x\}$. Cuando $x$ y $y$ son vecinos, también decimos que son adyacentes. La vecindad de un conjunto de vértices $A \subseteq G$, se define como: $N_{G}[A]=\bigcup_{x \in A} N_{G}[x]$.

Si $G$ es una gráfica y $x, y \in G$ denotamos por $d_{G}(x, y)$ a la distancia entre los vértices $x$ e $y$ en $G$. Si $A, B \subseteq V(G)$ definimos el conjunto de distancias de $A$ y $B$ en $G$, como $\mathcal{D}_{G}(A, B)=\left\{d_{G}(a, b): a \in A\right.$ y $\left.b \in B\right\}$. Cuando sea claro escribiremos solamente $d(x, y)$ y $\mathcal{D}(A, B)$ respectivamente.

El diámetro de $G$ se define $\operatorname{como} \operatorname{diam}(G)=\max \left\{d_{G}(x, y): x, y \in G\right\}$. Si $x \in G$ definimos el radio local de $G$ en $x$ como $\operatorname{radio}_{x}(G)=\max _{y \in G} d(x, y)$. Definimos el radio de $G$ como $\operatorname{radio}(G)=\min _{x \in G} \operatorname{radio}_{x}(G)$.

Si $x, y \in G$, decimos que $x$ domina a $y$ en $G$ si $N_{G}[x] \supseteq N_{G}[y]$. Sin embargo decimos que $y$ es un vértice dominado ( $\sin$ especificar quién es $x$ ) solamente cuando $y$ es dominado por algún vértice distinto $x \neq y$.

Un morfismo $f$ entre dos gráficas $A$ y $B$ es una función $f: V(A) \rightarrow V(B)$ tal que las imágenes de vértices adyacentes son adyacentes o iguales. En este caso escribimos simplemente $f: A \rightarrow B$. La categoría de las gráficas $\mathcal{G}$ es la categoría que tiene por objetos a todas las gráficas y por morfismos los recién descritos. En esta categoría el producto categórico de dos gráficas $A$ y $B$ es el producto fuerte $A \otimes B$ que tiene por vértices al producto cartesiano $V(A \otimes B)=V(A) \times V(B)$; dos vértices distintos $\left(a_{1}, b_{1}\right),\left(a_{2}, b_{2}\right) \in A \otimes B$ son adyacentes si y sólo si $a_{1}$ es adyacente o igual a $a_{2}$ y $b_{1}$ es adyacente o igual a $b_{2}$. El producto fuerte tiene muchísimas propiedades relevantes para el estudio de las gráficas iteradas de clanes. Una de las más importantes es:

Teorema 1.1 (Neumann-Lara [56]) Si $A$ y $B$ son gráficas, tenemos $k(A \otimes B) \cong$ $k(A) \otimes k(B)$.

Otra propiedad que usaremos es:

Teorema 1.2 (Neumann-Lara [54]) Si $\left(a_{1}, b_{2}\right),\left(a_{2}, b_{2}\right) \in A \otimes B$ entonces

$$
d_{A \bowtie B}\left(\left(a_{1}, b_{1}\right),\left(a_{2}, b_{2}\right)\right)=\max \left\{d_{A}\left(a_{1}, a_{2}\right), d_{B}\left(b_{1}, b_{2}\right)\right\}
$$

En particular $\operatorname{diam}(A \otimes B)=\max \{\operatorname{diam}(A), \operatorname{diam}(B)\}$. 
En ocasiones, vamos a requerir la noción de gráfica punteada. Una gráfica punteada es un par ordenado $(A, x)$ donde $A$ es una gráfica y $x$ es un vértice seleccionado de $A$. La suma punteada de dos gráficas punteadas $(A, x) \cdot(B, y)$ es la gráfica punteada que se obtiene al tomar la unión disjunta de $A$ y $B$ y luego identificar los vértices seleccionados $x$ y $y$. El vértice $x=y$ de $(A, x) \cdot(B, y)$ es su vértice seleccionado. También, hablaremos de la suma punteada $A \cdot B$ de dos gráficas $A$ y $B$ : el contexto hará claro cuales son los vértices de $A$ y $B$ que habrán de identificarse.

Algunas gráficas especiales serán de uso frecuente:

Denotamos por $\mathcal{C}_{n}$ al ciclo de $n$ vértices. $\mathcal{P}_{n}$ representará a la trayectoria de $n$ vértices. La gráfica completa (cualesquiera dos vértices son vecinos) de $n$ vértices será representada por $K_{n}$. El octaedro $n$-dimensional, $\mathcal{O}_{n}$, es la gráfica con $2 n$ vértices, cada uno de ellos de grado (=número de vecinos) $2 n-2$. Cuando $n=3$ decimos simplemente que se trata del octaedro.

Si $H$ y $G$ son gráficas, decimos que $G$ es localmente $H$, si para cualquier vértice $x \in G$ la subgráfica inducida $G\left[N_{G}(x)\right]$ es isomorfa a $H$. Si $\mathcal{H}=\left\{H_{1}, H_{2}, \ldots\right\}$ es una familia (no necesariamente finita) de gráficas, decimos que $G$ es localmente $\mathcal{H}$ si cada $G\left[N_{G}(x)\right]$ es isomorfa a alguna $H_{i} \in \mathcal{H}$. Decimos que $G$ es una gráfica localmente cíclica si $G$ es localmente $\left\{\mathcal{C}_{t}: t \geq 3\right\}$.

En su momento hablaremos también un poco sobre triangulaciones:

Por supuesto (ver por ejemplo [36,73]), una triangulación de un espacio topológico $X$ es un par ordenado $(C, f)$ donde $C$ es un complejo simplicial y $f:|C| \rightarrow X$ es un homeomorfismo que va de la realización geométrica $|C|$ de $C$ al espacio $X$. Cuando $X=|C|$ y $f=1_{C}$ y también cuando no haya confusión posible, diremos que $C$ es una triangulación.

El 1-esqueleto de un complejo simplicial $C$ es el subcomplejo de $C$ que consta de todos los simplejos de dimensión 0 ó 1. De manera natural, el 1-esqueleto de cualquier complejo se puede ver como una gráfica: los 0-simplejos son los vértices y los 1-simplejos son las aristas. Llamamos a esta gráfica la gráfica subyacente del complejo. En general, uno no puede reconstruir el complejo a partir del 1-esqueleto, pero nosotros consideraremos solamente triangulaciones de superficies y solamente triangulaciones en donde todo triángulo del 1-esqueleto es una cara (=2-simplejo). A una triangulación con estas características le llamamos triangulación de Whitney [79](1931). 
En el caso de tales triangulaciones, observamos que toda la información del complejo puede ser reconstruida a partir del 1-esqueleto. Dependiendo del contexto, consideraremos pues a una triangulación de Whitney como espacio topológico, como complejo simplicial, como el 1-esqueleto del complejo y/o como la gráfica subyacente, sin ser muy explícitos al respecto.

Notamos también que, con excepción del tetraedro $K_{4}$, toda triangulación de Whitney tiene la propiedad de que los clanes de la gráfica subyacente, son exactamente las caras de la triangulación. De ahí nuestro interés en este tipo de triangulaciones.

Las triangulaciones de Whitney han sido también llamadas triangulaciones limpias $[29,77]$.

Los siguientes dos resultados nos muestran que las triangulaciones de Whitney tienen sencillas caracterizaciones combinatorias. No hemos encontrado estos resultados publicados previamente, pero es probable que sean parte del folclore.

Teorema 1.3 (Larrión, Neumann-Lara y Pizaña [45]) G es la gráfica subyacente a una triangulación de Whitney de una superficie (cerrada) si y solamente si $G$ es localmente cíclica.

Teorema 1.4 (Larrión, Neumann-Lara y Pizaña [45]) G es la gráfica subyacente a una triangulación de Whitney de una superficie compacta (que puede tener borde) si y solamente si $G$ es localmente $\left\{\mathcal{C}_{t}, \mathcal{P}_{r}: t \geq 3, r \geq 2\right\}$.

Para la poca terminología de grupos que usaremos seguiremos a Rotman [72] y para categorías a Mac Lane [52].

\section{Resultados preliminares}

Resumimos en las siguientes dos subsecciones los resultados sobre gráficas clan convergentes y gráficas clan divergentes que usaremos más adelante. 


\section{Gráficas clan convergentes}

Una clase de gráficas que es sumamente importante en el estudio de las gráficas clan convergentes es la clase de las gráficas clan-Helly:

Definición 1.5 Decimos que una gráfica $G$ es clan-Helly si el conjunto de todos sus clanes $V(k(G))$ satisface la propiedad de Helly:

Helly: Para toda familia $\mathcal{F} \subseteq V(k(G))$, si cualesquiera dos elementos de $\mathcal{F}$ tienen intersección no vacía, entonces toda la familia tiene intersección no vacía.

Se sabe por Moon y Moser [53](1965) que una gráfica con $n$ vértices puede tener hasta $3^{m}, 4 \cdot 3^{m-1}(m \geq 1)$ ó $2 \cdot 3^{m}$ clanes, dependiendo de si $n=3 m, 3 m+1$ ó $3 m+2$. Una prueba independiente, puede ser obtenida usando los resultados de Hedman sobre gráficas densas [34]. Éstas, son cotas superiores que efectivamente se alcanzan. Cotas muy semejantes se pueden alcanzar incluso si se pide que la gráfica sea clan-Helly, simplemente agregando un nuevo vértice que sea vecino de todos los demás a cualquiera de las gráficas que alcanzan estas cotas [76]. Por esta razón es muy sorprendente que de hecho, se pueda verificar en tiempo polinomial si una gráfica dada es clan-Helly:

Teorema 1.6 (Szwarcfiter [75]) Una gráfica $G$ es clan-Helly si y solamente si todo triángulo extendido de $G$ es un cono.

Aquí un triángulo extendido de $G$ es la subgráfica inducida en $G$ por un conjunto de vértices que incluye los vértices de un triángulo $T$ junto con todos los vértices de $G$ que sean adyacentes a dos o más vértices del triángulo. Por supuesto, un cono es una gráfica en la que existe un vértice que es adyacente a todos los demás.

La clase de las gráficas clan-Helly es una de las primeras clases de gráficas que fueron reconocidas como $k$-cerradas [15], es decir que la gráfica de clanes de cualquier gráfica clan-Helly es a su vez clan-Helly.

Una de las razones por las que las gráficas clan-Helly son tan importantes en la teoría de gráficas de clanes es el siguiente famoso teorema debido Escalante: 
Teorema 1.7 (Escalante [15]) Sea $G$ una gráfica clan-Helly sea $H$ cualquier subgráfica inducida minimal de $G$ que satisfaga: Para toda $x \in G$ existe una $y \in H$ tal que $y$ domina a $x$. Entonces $k^{2}(G) \cong H$. En particular, toda gráfica clan-Helly es clan convergente de periodo 1 o 2 .

La mayoría de los artículos que estudian gráficas clan convergentes prueban que sus gráficas son a la larga clan-Helly $\left(k^{n}(G)\right.$ es clan-Helly para alguna $\left.n\right)$. Nosotros mismos usaremos esta idea en el capítulo 5. Una notable excepción es el propio artículo de Escalante [15], en donde se construyen gráficas $k$-periódicas de cualquier periodo: cuando el periodo es mayor que 2, la gráfica no puede ser clan-Helly.

Otros ejemplos de gráficas periódicas no clan-Helly se pueden construir fácilmente usando los relojes introducidos por Larrión y Neumann-Lara en [41]. Nosotros estudiaremos los relojes en el capítulo 3.

Bornstein y Szwarcfiter [8] construyen ejemplos interesantes de gráficas con defecto de Helly grande: Para toda $n$ existen gráficas donde $k^{n}(G)$ es clan-Helly, pero $k^{n-1}(G)$ no lo es. Estas gráficas son $k$-nulas, y por lo tanto, son a la larga clan-Helly, pero no es este hecho el que se usa para probar su convergencia.

Algunas interesantes consecuencias inmediatas del teorema de Escalante son:

Teorema 1.8 Para una gráfica clan-Helly $G$ las siguientes condiciones son equivalentes:

1. G es k-periódica.

2. $G \cong k^{2}(G)$.

3. G no tiene vértices dominados.

4. La función $*: G \rightarrow c(k(G))$ dada por $*(x)=\{Q \in k(G): x \in Q\}$ es de hecho un isomorfismo entre $G$ y $k^{2}(G)$.

Estos resultados hacen ver que los clanes de clanes (vértices en $k^{2}(G)$ ) se dividen en dos tipos: 
Definición 1.9 Si $G$ es una gráfica cualquiera y $Q \in k^{2}(G)$ es un clan de clanes de $G$, entonces decimos que $Q$ es una corbata de $G$ si $\cap Q=\varnothing$ y decimos que es una estrella de $G$ si $Q \neq \varnothing$.
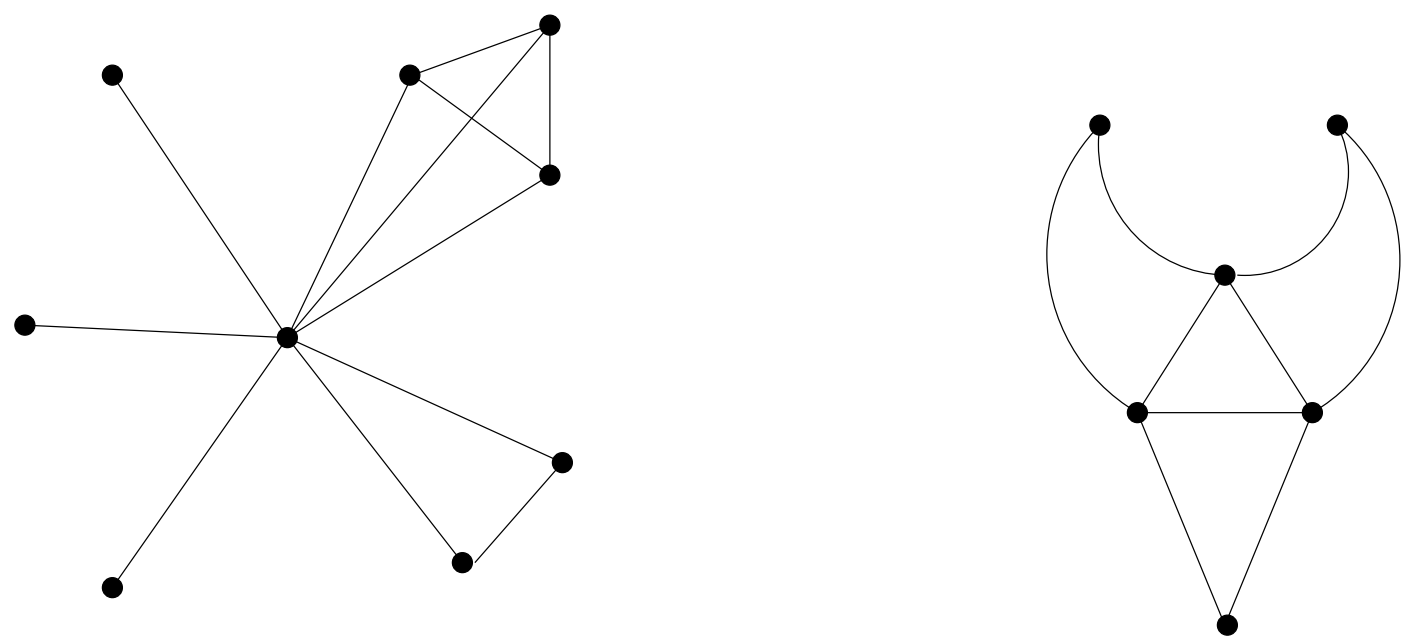

Figura 1.1: Una estrella y una corbata

Claramente, una gráfica es clan-Helly si y sólo si no tiene corbatas. Sabemos entonces, por el teorema de Escalante, que si una gráfica es $k$-divergente tiene que tener corbatas. El recíproco, sin embargo, es falso. Un ejemplo de esto es la gráfica de la derecha en la figura 1.1; ejemplos no triviales pueden ser encontrados en [8].

También, si $x$ es un vértice de $G$ observamos que $*(x)$ (tal y como se define en el teorema anterior) es una completa de $k(G)$, pero en general puede no ser un clan de $k(G)$. Sin embargo, para cada $x \in G$ siempre podemos escoger un clan $\widehat{*(x)}$ de $k(G)$ que contenga a $*(x)$. Lo interesante es que no importa cómo haga uno esta elección, la función resultante es un morfismo de $G$ a $k^{2}(G)$. Abreviamos $*(x)$ como $x^{*}$ y decimos que el morfismo $x \mapsto x^{*}$ es el morfismo estrella de $G$ en $k^{2}(G)$. Cuando queremos enfatizar que este morfismo no es único diremos que es un morfismo estrella.

Note que si $*(x)$ es clan (de clanes), también es estrella. Por otro lado $x^{*}$ puede ser una estrella o una corbata, pero toda estrella $Q \in k^{2}(G)$ es de la forma $Q=x^{*}=*(x)$ para alguna $x \in G$. 


\section{Gráficas clan divergentes}

Como ya se dijo antes, los octaedros $n$-dimensionales fueron los primeros ejemplos conocidos de gráficas clan divergentes. Desde entonces otro par de familias de ejemplos han sido encontradas, a saber los relojes $[38,41]$ y ciertas triangulaciones de Whitney del toro [39] y de la botella de Klein [40]. Estos ejemplos son primitivos en el sentido que se tienen pruebas directas de su divergencia que involucran el cálculo explícito de todas sus gráficas iteradas de clanes. Estos, y los que se pueden construir a partir de estos usando el producto fuerte $\otimes$ (ver Teorema 1.1) son los únicos ejemplos primitivos que se conocen.

Las triangulaciones de Whitney del toro y los relojes tienen la virtud de tener un crecimiento lineal. Ello permite calcular y estudiar muchas de sus gráficas iteradas de clanes para así poder desentrañar sus misterios. En el caso de los octaedros, si bien el crecimiento es explosivo y son pocas las gráficas iteradas de clanes que pueden ser efectivamente calculadas, las gráficas que se obtienen son siempre octaedros $m$ dimensionales y no es difícil aún con pocos ejemplos entender lo que sucede.

Sin embargo, las gráficas iteradas de clanes de una gráfica dada exhiben en general un comportamiento muy complejo, en apariencia caótico. Por ejemplo, la sucesión de órdenes de las gráficas iteradas de clanes del icosaedro es: 12, 20, 32, 92, 472, 44644, ?? Este tipo de ejemplo es frecuente: La computadora es capaz de calcular solamente unas pocas gráficas iteradas de clanes debido al crecimiento explosivo del orden de las gráficas. En casos como éste son pocas las gráficas iteradas que uno tiene a mano para estudiar y tratar de entender qué es lo que ocurre. En el caso del icosaedro, aún estas pocas gráficas iteradas de clanes son difíciles de entender y la comprensión de la $n$-ésima iteración da poca luz sobre la siguiente.

Concluimos que para estudiar la clan divergencia hacen falta técnicas y criterios que permitan inferir la clan divergencia de las gráficas sin tener que calcular todas sus gráficas iteradas de clanes. Así por ejemplo, nosotros probaremos en el capítulo 4 que el icosaedro es clan divergente sin saber siquiera cuál es el orden de su sexta gráfica de clanes.

Todas las técnicas conocidas a la fecha involucran un enfoque categórico y clases de morfismos especiales en esas categorías que tienen la propiedad de heredar la clan divergencia, es decir, cada vez que $A$ y $B$ son gráficas en la categoría y $\varphi: A \rightarrow B$ 
es uno de estos morfismos especiales, entonces tenemos que si $A$ es $k$-divergente, también lo es $B$. Listaremos a continuación los teoremas que se tienen en ese sentido.

Sean $A$ y $B$ dos gráficas, decimos que un morfismo entre ellas $\varphi: A \rightarrow B$ es una retracción si existe un morfismo $\tau: B \rightarrow A$ tal que $\varphi \circ \tau=1_{B}$. En este caso, decimos que $\tau$ es una sección y que $B$ es un retracto de $A$. En este caso la clase de morfismos que heredan la clan divergencia es la clase de las secciones:

Teorema 1.10 (Teorema de la retracción, Neumann-Lara [56]) Si B es un retracto de $A$, entonces, $k^{n}(B)$ es un retracto de $k^{n}(A)$ para toda $n$. En particular, Si $B$ es clan divergente $A$ también lo es y si $A$ es clan nula $B$ también lo es.

Como puede verse en [37], este teorema permite estudiar amplísimas clases de gráficas clan divergentes.

Si $B$ es una subgráfica de $A$ y podemos obtener $B$ a partir de $A$ eliminando vértices dominados uno a uno, diremos que $A$ se retrae fuertemente a $B$ y que $B$ es un retracto fuerte de $A$. Note que $A$ puede no retraerse fuertemente a otra subgráfica $C$ aún si $B \cong C$. Claramente, este es un caso particular de la retracción y si $\iota: B \rightarrow A$ es la inclusión, diremos que cualquier morfismo que tenga a $\iota$ como sección, es una retracción fuerte. Por supuesto, el teorema anterior vale para las retracciones fuertes, pero de hecho, podemos decir mucho más para las retracciones fuertes:

Teorema 1.11 (Teorema de la retracción fuerte, Frías y Neumann-Lara [16,17]) Si $x$ es un vértice dominado de de una gráfica $A$, entonces, $A$ y $A-\{x\}$ tienen el mismo $k$-carácter. Es decir, $A$ es $k$-divergente si y sólo si $A-\{x\}$ lo es y $A$ es $k-n u l a ~ s i$ y sólo si $A-\{x\}$ lo es. En particular, si $A$ se retrae fuertemente a $B$, entonces $A$ y $B$ tienen el mismo $k$-carácter.

Un caso particular del teorema anterior fue probado por Prisner en 1992:

Teorema 1.12 (Prisner [64]) Si A se retrae fuertemente a un punto, A es k-nula. 
Otro instancia particular de la retracción es cuando $A \cong B \otimes C$. Aquí, tanto $B$ como $C$ son retractos de $A$. También en este caso, usando el teorema 1.1, se puede decir más sobre $A$ que lo que nos ofrece el teorema de la retracción. En particular, $A$ es $k$-divergente si y sólo si $B$ ó $C$ lo son y $A$ es $k$-nula si y sólo si $B$ y $C$ lo son.

Otra interesante clase de morfismos que heredan la clan divergencia es la clase de los cubrimientos triangulares: Decimos que $\varphi: A \rightarrow B$ es un cubrimiento triangular si para cada $x \in A$, la restricción de $\varphi$ a $N_{A}[x]$ es un isomorfismo sobre $N_{B}[\varphi(x)]$. También diremos que $A$ cubre triangularmente a $B$. Los cubrimientos triangulares también son llamados isomorfismos locales. Si $\varphi$ es un cubrimiento triangular, se sabe que $\varphi^{-1}(x)$ tiene la misma cardinalidad para toda $x \in B$, llamamos a este número el número de hojas del cubrimiento triangular.

Teorema 1.13 (Teorema del cubrimiento triangular, Larrión y Neumann-Lara [40]) Si A cubre triangularmente a $B$ con $h$ hojas, entonces, $k^{n}(A)$ cubre triangularmente a $k^{n}(B)$ con h hojas para toda $n$. En particular, $A$ y $B$ tienen el mismo $k$-carácter.

El resultado anterior junto con la clan divergencia de ciertas triangulaciones del toro, fue usado en [40] para probar que toda gráfica localmente $\mathcal{C}_{6}$ es clan divergente.

Una clase más de morfismos que heredan la clan divergencia es descrita en el hermoso trabajo sobre gráficas expansivas de Neumann-Lara [55]. No lo ponemos aquí porque dedicaremos el capítulo 4 por entero a desarrollar y generalizar estas ideas.

Una vez aclarado el panorama general, cabe enfatizar la importancia de los ejemplos primitivos: Sin ellos, todas las técnicas y criterios desarrollados no constituirían más que una teoría vacía. Los ejemplos primitivos son pues fundamentales. 


\section{Capítulo 2}

\section{Cimientos}

Los cimientos fueron introducidos por Bornstein y Szwarcfiter en [8] y han sido utilizados en $[9,60,62]$. Han mostrado ser de suma utilidad en el estudio de las propiedades métricas de las gráficas iteradas de clanes, y en particular para el estudio del comportamiento del diámetro y el radio bajo el operador de clanes. En este capítulo presentaremos nuestras aportaciones a la teoría de cimientos siendo la fórmula de la distancia (teorema 2.17) la más fundamental. También obtendremos algunas conclusiones interesantes:

Daremos una nueva familia de gráficas $\left\{G_{n}\right\}$ con la propiedad $\operatorname{diam}\left(k^{m}(G)\right)=$ $\operatorname{diam}(G)+m$ para toda $0 \leq m \leq n$, dando así una nueva prueba al teorema del diámetro de Bornstein y Szwarcfiter [9](1998) (ver teorema 2.21), antes conocida como la conjetura del diámetro de Peyrat, Rall y Slater [59](1986).

También, estudiaremos el problema de decidir si existe una gráfica $G$ que satisfaga $\operatorname{diam}\left(k^{n}(G)\right)=\operatorname{diam}(G)+n$ para toda $n \geq 0$ y obtendremos varias conclusiones sobre la posible existencia de tal gráfica.

\section{Distancias en $G$ y en $k(G)$}

Empecemos por un teorema elemental que fue originalmente escrito para mi trabajo sobre la clan divergencia del icosaedro [61]: 
Teorema 2.1 Sea $G$ una gráfica y $Q, Q^{\prime} \in k(G)$ con $Q \neq Q^{\prime}$. Entonces

$$
d_{k(G)}\left(Q, Q^{\prime}\right)=\min \mathcal{D}_{G}\left(Q, Q^{\prime}\right)+1
$$

Prueba. Sea $r=\min \mathcal{D}\left(Q, Q^{\prime}\right)$ y $s=d\left(Q, Q^{\prime}\right)$. Sea $\left(x_{0}, x_{1}, \ldots, x_{r}\right)$ una trayectoria en $G$ con $x_{0} \in Q$ y $x_{r} \in Q^{\prime}$. Tomemos $Q_{i} \in V(k(G))$ tal que $\left\{x_{i}, x_{i+1}\right\} \subseteq Q_{i}$ para $i=0,1, \ldots, r-1$. Entonces $\left(Q, Q_{0}, Q_{1}, \ldots, Q_{r-1}, Q^{\prime}\right)$ es un camino en $k(G)$ que muestra que $d\left(Q, Q^{\prime}\right)=s \leq r+1$.

Ahora sea $\left(Q_{0}, Q_{1}, \ldots, Q_{s}\right)$ una trayectoria de longitud mínima en $k(G)$ entre $Q_{0}=Q$ y $Q_{s}=Q^{\prime}$. Tomemos ahora $x_{i} \in Q_{i} \cap Q_{i+1}$ para $i=0,1, \ldots, s-1$. Luego, $\left(x_{0}, x_{1}, \ldots, x_{s-1}\right)$ es un camino en $G$, que demuestra que $d\left(x_{0}, x_{s-1}\right) \leq s-1$. Como $x_{0} \in Q$ y $x_{s-1} \in Q^{\prime}$, se sigue que $r+1=\min \mathcal{D}\left(Q, Q^{\prime}\right)+1 \leq d\left(x_{0}, x_{s-1}\right)+1 \leq s$.

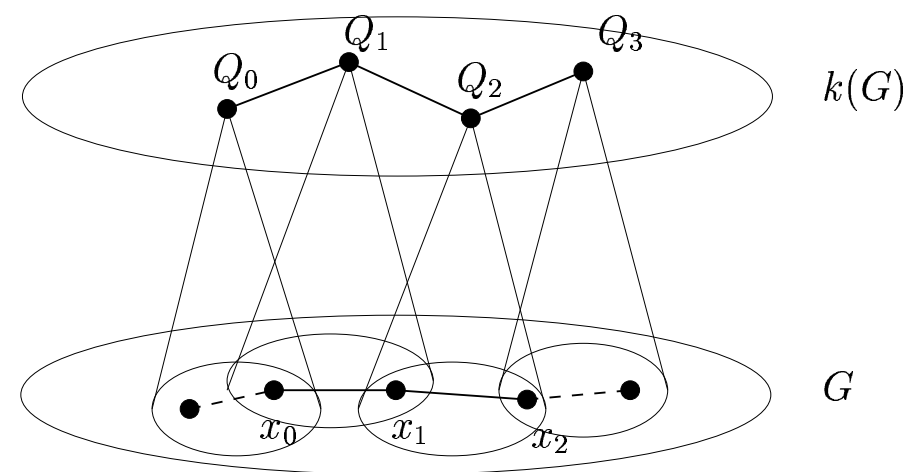

Figura 2.1: Trayectorias en $G$ y en $k(G)$

Note que para toda gráfica $G$ y cualesquiera dos clanes $Q, Q^{\prime} \in V(k(G))$, tenemos $\left|\mathcal{D}\left(Q, Q^{\prime}\right)\right| \leq 3$ y $\mathcal{D}\left(Q, Q^{\prime}\right) \neq\{1\}$. Si $G$ tiene al menos dos vértices en cada componente conexa, entonces $\mathcal{D}\left(Q, Q^{\prime}\right) \neq\{0\}$ y por lo tanto $\mathcal{D}\left(Q, Q^{\prime}\right)=\{0,1\}$ si y sólo si $d\left(Q, Q^{\prime}\right)=0$ (i.e. $\left.Q=Q^{\prime}\right)$. Observe también, que si $Q$ y $Q^{\prime}$ son cualesquiera dos clanes, tenemos $\max \mathcal{D}\left(Q, Q^{\prime}\right) \leq \min \mathcal{D}\left(Q, Q^{\prime}\right)+2$ (ver figura 2.1).

Como dijimos el teorema es elemental. La prueba es directa. Sin embargo, es un teorema sintetizador en el sentido de que recoge la esencia de una argumentación que ha sido usada una y otra vez para probar distintos teoremas y lemas en no pocos artículos: Los catorce corolarios que se listan a continuación, son una muestra de ello. Cada uno de estos teoremas en sus respectivos artículos incluía una prueba, todas estas pruebas con la misma idea subyacente, la misma del teorema 2.1. La idea no 
es nueva, la prueba no es nueva, lo que es nuevo es la forma que toma el enunciado del teorema 2.1. Esta forma permite probar cualquiera de los corolarios que se listan a continuación sin mayor esfuerzo. No podemos decir lo mismo de ninguno de los catorce teoremas siguientes aunque hemos de reconocer que Dutton y Brigham se acercaron bastante.

Corolario 2.2 (Hedman [33, teorema 4.2]) Si G es una gráfica conexa,

$$
\operatorname{diam}(k(G)) \geq \operatorname{diam}(G)-1 .
$$

Prueba. Inmediato

Decimos que una gráfica $G$ es una gráfica de tiempo, si existe una función inyectiva $f: V(G) \rightarrow \mathbb{R}$ tal que para cada par de vértices distintos $u, v \in V(G)$ tenemos que $\{u, v\} \in E(G)$ si y sólo si $|f(u)-f(v)| \leq 1$.

Corolario 2.3 (Hedman [33, lema 4.3]) Si G es una gráfica de tiempo conexa, entonces $\operatorname{diam}(G)>\operatorname{diam}(k(G))$ [Excepto, claro, cuando $G$ es trivial].

Prueba. Sean $a, b \in G$ tal que $f(a) \leq f(x) \leq f(b)$ para toda $x \in G$. Calcule la distancia entre los únicos clanes $A$ y $B$ que satisfacen $a \in A$ y $b \in B$.

Corolario 2.4 (Hedman [35, teorema 3.1]) Si $G$ es una gráfica conexa, $\operatorname{diam}(G)+$ $1 \geq \operatorname{diam}(k(G)) \geq \operatorname{diam}(G)-1$.

Prueba. Inmediato.

Corolario 2.5 (Teorema del diámetro de Hedman [35, teorema 4.1]) Para una gráfica conexa $G, \operatorname{diam}(G)+n \geq \operatorname{diam}\left(k^{n}(G)\right) \geq \operatorname{diam}(G)-n$.

Prueba. Inmediato del corolario anterior.

Siguiendo a Balakrishnan y Paulraja [3], decimos que dos clanes $A$ y $B$ son diametrales si $\operatorname{diam}(G) \in \mathcal{D}(A, B)$. 
Corolario 2.6 (Balakrishnan y Paulraja, [3, teorema 1]) Para una gráfica conexa $G$, $\operatorname{diam}(k(G))=\operatorname{diam}(G)+1$ si y sólo si existe un par de clanes diametrales [distintos] de tipo 1 en $G$.

Prueba. Dos clanes diametrales $A$ y $B$ son de tipo 1 si $\min \mathcal{D}(A, B)=\operatorname{diam}(G)$.

Corolario 2.7 (Balakrishnan y Paulraja, [3, teorema 2]) Sea G una gráfica conexa. Entonces $\operatorname{diam}(G)=\operatorname{diam}(k(G))$ si y sólo si existe un par de clanes diametrales [distintos] de tipo 2 en $G$ y no existe un par de clanes diametrales [distintos] de tipo 1 en $G$ [o bien $G$ es la gráfica trivial].

Prueba. $A$ y $B$ son clanes diametrales de tipo 2 si $\min \mathcal{D}(A, B)=\operatorname{diam}(G)-1$.

Corolario 2.8 (Balakrishnan y Paulraja, [3, teorema 3]) Sea G una gráfica conexa. Entonces $\operatorname{diam}(G)-1=\operatorname{diam}(k(G))$ si y sólo si cada par de clanes diametrales es de tipo 3 [o bien $G$ es una completa no trivial].

Prueba. $A$ y $B$ son clanes diametrales de tipo $3 \sin \min \mathcal{D}(A, B)=\operatorname{diam}(G)-2$.

Corolario 2.9 (Peyrat, Rall y Slater [59, teorema 2]) Sea $G$ una gráfica de diámetro d. $k(G)$ tiene diámetro $d+1$ si y sólo si $G$ tiene dos clanes $C$ y $D$ tal que la distancia $d(x, y)=d$ para toda $x \in C, y \in D$.

Prueba. Inmediato.

Corolario 2.10 (Dutton y Brigham [14, lema]) Sean $C$ y $C^{\prime}$ vértices arbitrarios de $k(G)$ con $v$, $v^{\prime}$ vértices arbitrarios de $G$ en $C$ y $C^{\prime}$ respectivamente. Entonces $d_{k(G)}\left(C, C^{\prime}\right)-1 \leq d_{G}\left(v, v^{\prime}\right) \leq d_{k(G)}\left(C, C^{\prime}\right)+1$.

Prueba. Inmediato del teorema 2.1 y la observación que le sigue.

Dutton y Brigham presentan el siguiente resultado como un corolario sin demostración explícita de su lema (corolario anterior). En mi opinión una prueba es necesaria: 
Corolario 2.11 (Dutton y Brigham [14, corolario 1(b)]) Para cualquier gráfica $G$, $\operatorname{radio}(G)-1 \leq \operatorname{radio}(k(G)) \leq \operatorname{radio}(G)+1$.

Prueba. Se usará el corolario anterior en las desigualdades centrales: Primero tomemos $x, y \in G$ y $P, Q \in k(G)$ de tal forma que: $\operatorname{radio}_{x}(G)=\operatorname{radio}(G), x \in P$, $\operatorname{radio}_{P}(k(G))=d(P, Q)$ y $y \in Q$. Entonces

$$
\operatorname{radio}(k(G)) \leq d(P, Q) \leq d(x, y)+1 \leq \operatorname{radio}(G)+1
$$

Si ahora tomamos $x, y \in G$ y $P, Q \in k(G)$ de forma que: $\operatorname{radio}_{P}(k(G))=\operatorname{radio}(k(G))$, $x \in P, \operatorname{radio}_{x}(G)=d(x, y)$ y $y \in Q$. Entonces

$$
\operatorname{radio}(k(G)) \geq d(P, Q) \geq d(x, y)-1 \geq \operatorname{radio}(G)-1
$$

Corolario 2.12 (Dutton y Brigham [14, corolario 2]) $S i d_{k(G)}\left(C, C^{\prime}\right)=m$, existen vértices $v \in C$ y $v^{\prime} \in C^{\prime}$ tal que $d_{G}\left(v, v^{\prime}\right)=m-1$ [Siempre y cuando $C \neq C^{\prime}$, i.e. $m>0]$.

Prueba. Inmediato.

Corolario 2.13 (Dutton y Brigham [14, teorema 2]) Para cualquier gráfica $G$, se tiene que: $\operatorname{diam}(k(G))=\operatorname{diam}(G)-1$ si y sólo si para cada par de clanes $C$ y $C^{\prime}$ existe un vértice $v \in C$ y un vértice $v^{\prime} \in C^{\prime}$ tal que $d_{G}\left(v, v^{\prime}\right) \leq \operatorname{diam}(G)-2$.

Prueba. Inmediato.

Corolario 2.14 (Dutton y Brigham [14, teorema 3]) Para cualquier gráfica $G$, se tiene que: $\operatorname{radio}(k(G))=\operatorname{radio}(G)+1$ si y sólo si, para cada clan $C$ existe un clan asociado $C^{\prime}$ tal que $d_{G}\left(v, v^{\prime}\right) \geq \operatorname{radio}(G)$, para toda $v \in C$ y $v^{\prime} \in C^{\prime}$.

Prueba. Inmediato del teorema 2.1 y el corolario 2.11. 
Corolario 2.15 (Dutton y Brigham [14, teorema 4]) Para cualquier gráfica $G$, se tiene que: $\operatorname{radio}(k(G))=\operatorname{radio}(G)-1$ si y sólo si existe un clan $C$ tal que para cada clan $C^{\prime}$ existen vértices $v \in C$ y $v^{\prime} \in C^{\prime}$ con $d_{G}\left(v, v^{\prime}\right) \leq \operatorname{radio}(G)-2$.

Prueba. Inmediato del teorema 2.1 y el corolario 2.11.

\section{La fórmula de la distancia}

Los cimientos que definiremos a continuación han sido sumamente útiles en el estudio del diámetro de las gráficas y en el estudio del defecto de Helly. Los cimientos fueron introducidos (con diferente nombre y diferente notación) por Bornstein y Szwarcfiter en $[8]$ y han sido utilizados en $[9,60,62]$.

Definición 2.16 Si $G$ es una gráfica $Q \in V\left(k^{n}(G)\right)$ definimos recursivamente el cimiento de $Q$ en $G, \mathcal{B}_{G}(Q)$, de la siguiente manera:

$$
\mathcal{B}_{G}(Q)= \begin{cases}Q & \text { si } n=1 \\ \bigcup_{q \in Q} \mathcal{B}_{G}(q) & \text { si } n>1\end{cases}
$$

La figura 2.2 ilustra esta definición.

Así, por ejemplo, si $Q \in k^{2}(G)$, entonces $\mathcal{B}_{G}(Q)$ es un conjunto de vértices de $G$, pero $\mathcal{B}_{k(G)}(Q)$ es un conjunto de vértices de $k(G)$ (clanes de $G$ ). Para simplificar la notación, a veces escribimos $\mathcal{B}_{m}(Q)$ en lugar de $\mathcal{B}_{k^{m}(G)}(Q)$ y también tomamos $\mathcal{B}(Q)=\mathcal{B}_{0}(Q)=\mathcal{B}_{G}(Q)$.

En la sección anterior, probamos el teorema 2.1 y señalamos la multitud de consecuencias que tiene. El siguiente teorema es una generalización incluso más útil que aquél.

Teorema 2.17 (La fórmula de la distancia) Sean $Q_{1}$ y $Q_{2}$ vértices de $k^{n}(G)$. Entonces:

$$
\max \mathcal{D}\left(\mathcal{B}\left(Q_{1}\right), \mathcal{B}\left(Q_{2}\right)\right)-n \leq d\left(Q_{1}, Q_{2}\right) \leq \min \mathcal{D}\left(\mathcal{B}\left(Q_{1}\right), \mathcal{B}\left(Q_{2}\right)\right)+n
$$




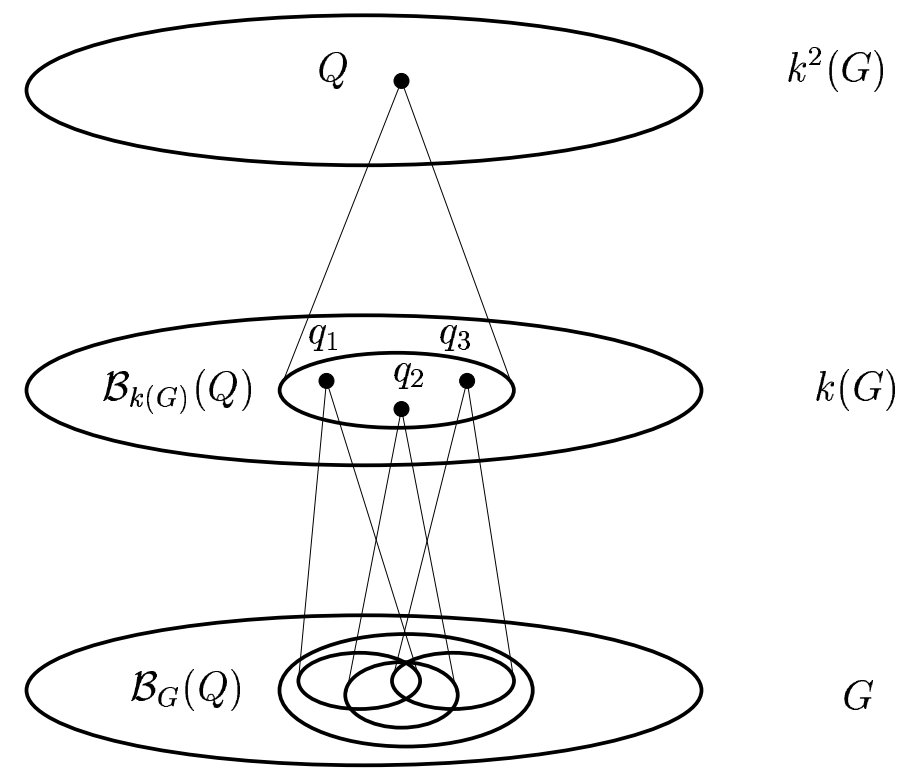

Figura 2.2: Cimientos

Si además $\mathcal{B}\left(Q_{1}\right) \cap \mathcal{B}\left(Q_{2}\right)$ no contiene (como subconjunto) a ningún clan de $G$, entonces:

$$
d\left(Q_{1}, Q_{2}\right)=\min \mathcal{D}\left(\mathcal{B}\left(Q_{1}\right), \mathcal{B}\left(Q_{2}\right)\right)+n
$$

Prueba. Consideremos el caso base $n=1$ :

Si $Q_{1}=Q_{2}$ el resultado es inmediato.

Supongamos entonces que $Q_{1} \neq Q_{2}$.

El teorema 2.1, nos dice que $d\left(Q_{1}, Q_{2}\right)=\min \mathcal{D}\left(\mathcal{B}\left(Q_{1}\right), \mathcal{B}\left(Q_{2}\right)\right)+1$. Obviamente $\max \mathcal{D}\left(\mathcal{B}\left(Q_{1}\right), \mathcal{B}\left(Q_{2}\right)\right) \leq \min \mathcal{D}\left(\mathcal{B}\left(Q_{1}\right), \mathcal{B}\left(Q_{2}\right)\right)+2$. Luego:

$$
\max \mathcal{D}\left(\mathcal{B}\left(Q_{1}\right), \mathcal{B}\left(Q_{2}\right)\right)-1 \leq \min \mathcal{D}\left(\mathcal{B}\left(Q_{1}\right), \mathcal{B}\left(Q_{2}\right)\right)+1=d\left(Q_{1}, Q_{2}\right)
$$

Supongamos ahora que $n \geq 2$ y apliquemos inducción:

$$
\begin{aligned}
d_{k^{n}(G)}\left(Q_{1}, Q_{2}\right) & +(n-1) \geq \max \mathcal{D}_{k(G)}\left(\mathcal{B}_{k(G)}\left(Q_{1}\right), \mathcal{B}_{k(G)}\left(Q_{2}\right)\right) \\
& =\max \left\{d_{k(G)}\left(P_{1}, P_{2}\right): P_{1} \in \mathcal{B}_{k(G)}\left(Q_{1}\right), P_{2} \in \mathcal{B}_{k(G)}\left(Q_{2}\right)\right\} \\
& \geq \max \left\{\max \mathcal{D}\left(\mathcal{B}\left(P_{1}\right), \mathcal{B}\left(P_{2}\right)\right)-1: P_{1} \in \mathcal{B}_{k(G)}\left(Q_{1}\right), P_{2} \in \mathcal{B}_{k(G)}\left(Q_{2}\right)\right\} \\
& =\max \mathcal{D}_{G}\left(\mathcal{B}\left(Q_{1}\right), \mathcal{B}\left(Q_{2}\right)\right)-1
\end{aligned}
$$


Similarmente:

$$
\begin{aligned}
d_{k^{n}(G)}\left(Q_{1}, Q_{2}\right) & -(n-1) \leq \min \mathcal{D}_{k(G)}\left(\mathcal{B}_{k(G)}\left(Q_{1}\right), \mathcal{B}_{k(G)}\left(Q_{2}\right)\right) \\
& \left.=\min \left\{d_{k(G)}\left(P_{1}, P_{2}\right): P_{1} \in \mathcal{B}_{k(G)}\left(Q_{1}\right), P_{2} \in \mathcal{B}_{k(G)}\left(Q_{2}\right)\right)\right\} \\
& \left.\leq \min \left\{\min \mathcal{D}\left(\mathcal{B}\left(P_{1}\right), \mathcal{B}\left(P_{2}\right)\right)+1: P_{1} \in \mathcal{B}_{k(G)}\left(Q_{1}\right), P_{2} \in \mathcal{B}_{k(G)}\left(Q_{2}\right)\right)\right\} \\
& =\min \mathcal{D}_{G}\left(\mathcal{B}\left(Q_{1}\right), \mathcal{B}\left(Q_{2}\right)\right)+1
\end{aligned}
$$

Cuando $\mathcal{B}_{G}\left(Q_{1}\right) \cap \mathcal{B}_{G}\left(Q_{2}\right)$ no contiene a ningún clan de $G$, se sigue que $\mathcal{B}_{k(G)}\left(Q_{1}\right) \cap$ $\mathcal{B}_{k(G)}\left(Q_{2}\right)=\varnothing$, en particular, esta intersección no contiene a ningún clan de $k(G)$ y $P_{1} \neq P_{2}$ para cualesquiera $P_{1} \in \mathcal{B}_{k(G)}\left(Q_{1}\right)$ y $P_{2} \in \mathcal{B}_{k(G)}\left(Q_{2}\right)$. Así que sólo hay que repetir el último argumento cambiando cada ' $\leq$ ' por un ' $=$ '.

Usando el teorema anterior, se pueden dar nuevas pruebas para el lema 1 de [8] y para el teorema 3 de [9].

La figura 2.3 nos puede dar una idea de qué tan buenas son las cotas. Tomando $G=\mathcal{P}_{5}$, tenemos un dibujo de $G$ y sus primeras 3 gráficas de clanes. Si tomamos los vértices $q_{1}^{2}$ y $q_{3}^{2}$, el teorema anterior nos dice que:

$$
2=\max \mathcal{D}\left(\mathcal{B}\left(q_{1}^{2}\right), \mathcal{B}\left(q_{3}^{2}\right)\right)-2 \leq d\left(q_{1}^{2}, q_{3}^{2}\right) \leq \min \mathcal{D}\left(\mathcal{B}\left(q_{1}^{2}\right), \mathcal{B}\left(q_{3}^{2}\right)\right)+2=2
$$

Si observamos que sus cimientos $\mathcal{B}_{G}\left(q_{1}^{2}\right)=\left\{x_{1}, x_{2}, x_{3}\right\}$ y $\mathcal{B}_{G}\left(q_{3}^{2}\right)=\left\{x_{3}, x_{4}, x_{5}\right\}$ sólo se intersectan en un vértice, el teorema de hecho nos da la garantía de que podremos conocer el valor exacto de la distancia entre $q_{1}^{2}$ y $q_{3}^{2}$. Por supuesto, cuando los cimientos se intersectan demasiado las cotas son menos buenas: $1=4-3 \leq d\left(q_{1}^{3}, q_{2}^{3}\right) \leq$ $0+3=3$. Observamos sin embargo, que cuando es posible calcular cimientos y distancias con respecto a un nivel adecuado (en este caso, por ejemplo calculando los cimientos respecto a $k^{2}(G)$, tenemos $\left.1=2-1 \leq d\left(q_{1}^{3}, q_{2}^{3}\right) \leq 0+1=1\right)$ siempre es posible conocer el valor exacto de la distancia que se busca. También hay casos en donde la cota inferior falla, pero no para esta $G$.

Para poder continuar con el estudio de los cimientos y su relación con las distancias en las gráficas iteradas de clanes necesitamos la noción de extensión de morfismos:

Si $\varphi_{0}: H \rightarrow G$ es un morfismo de gráficas, entonces, decimos que $\varphi_{1}: k(H) \rightarrow$ $k(G)$ es una extensión de $\varphi_{0}$ a $k(H)$ si $\varphi_{0}(Q) \subseteq \varphi_{1}(Q)$ para toda $Q \in V(k(H))$. Similarmente, decimos que $\varphi_{n}: k^{n}(H) \rightarrow k^{n}(G)$ es una extensión de $\varphi_{0}$ a $k^{n}(H)$ si 


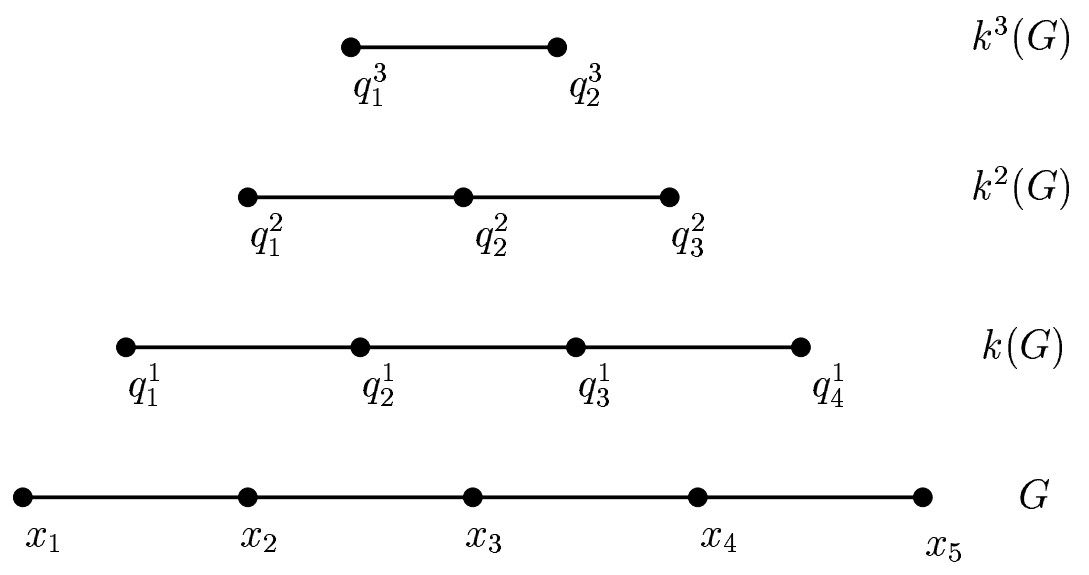

Figura 2.3: Distancias en $G$ y $k^{n}(G)$

existen morfismos $\varphi_{1}, \varphi_{2} \ldots, \varphi_{n-1}$ tal que $\varphi_{i+1}: k^{i+1}(H) \rightarrow k^{i+1}(G)$ es una extensión de $\varphi_{i}: k^{i}(H) \rightarrow k^{i}(G)$ a $k\left(k^{i}(H)\right)$ para $i=0,1, \ldots, n-1$.

Claramente, todo morfismo de gráficas tiene al menos una extensión a cada nivel (a cada $k^{n}(H)$ ). En general, estas extensiones pueden no conservar muchas de las características del morfismo original, en particular, una extensión de un morfismo no trivial bien puede ser trivial (su imagen es un punto). Sin embargo tenemos el siguiente teorema:

Teorema 2.18 Sea $\varphi_{0}: H \rightarrow G$ un morfismo de gráficas y $\varphi_{n}: k^{n}(H) \rightarrow k^{n}(G)$ cualquier extensión de $\varphi_{0}$ a $k^{n}(H)$. Tome $Q \in k^{n}(H)$. Entonces:

$$
\varphi_{0}\left(\mathcal{B}_{H}(Q)\right) \subseteq \mathcal{B}_{G}\left(\varphi_{n}(Q)\right)
$$

Prueba. El caso base es inmediato. Sea $\varphi_{n-1}: k^{n-1}(H) \rightarrow k^{n-1}(G)$ una extensión de $\varphi_{0}$ tal que $\varphi_{n}$ es una extensión de $\varphi_{n-1}$. Por hipótesis de inducción, para cualquier $q \in k^{n-1}(H)$, tenemos $\varphi_{0}\left(\mathcal{B}_{H}(q)\right) \subseteq \mathcal{B}_{G}\left(\varphi_{n-1}(q)\right)$. Luego, para $Q \in k^{n}(H)$ tenemos $\varphi_{0}\left(\mathcal{B}_{H}(Q)\right)=\varphi_{0}\left(\bigcup_{q \in Q} \mathcal{B}_{H}(q)\right)=\bigcup_{q \in Q} \varphi_{0}\left(\mathcal{B}_{H}(q)\right) \subseteq \bigcup_{q \in Q} \mathcal{B}_{G}\left(\varphi_{n-1}(q)\right)=$ $\mathcal{B}_{G}\left(\varphi_{n-1}(Q)\right) \subseteq \mathcal{B}_{G}\left(\varphi_{n}(Q)\right)$.

Cuando $H$ es una subgráfica inducida de $G$, es posible que un clan iterado de $H$ sea a su vez un clan iterado de $G$. Las extensiones nos permiten discernir si ese es el caso: 
Teorema 2.19 Sea $H$ una subgráfica inducida de $G$. Sea $\varphi_{0}: H \rightarrow G$ la inclusión. Sea $\varphi_{n}: k^{n}(H) \rightarrow k^{n}(G)$ cualquier extensión de $\varphi_{0}$ a $k^{n}(H)$ y sea $Q \in k^{n}(H)$. Entonces son equivalentes:

1. $\varphi_{n}(Q) \in k^{n}(H)$

2. $\varphi_{n}(Q)=Q$

3. $Q \in k^{n}(G)$

Prueba. En el caso $n=1$, las equivalencias son triviales. Probaremos el paso inductivo:

(2) $\Rightarrow(\mathbf{1})$ : trivial.

$(2) \Rightarrow(3)$ : trivial.

(1) $\Rightarrow(2)$ : Sea $Q=\left\{q_{1}, \ldots, q_{s}\right\}$. Entonces

$$
\varphi_{n}(Q)=\left\{\varphi_{n-1}\left(q_{1}\right), \ldots, \varphi_{n-1}\left(q_{s}\right), p_{1}, \ldots, p_{r}\right\}
$$

para algunas $p_{i} \in k^{n-1}(G)$. Por hipótesis $\varphi_{n}(Q) \in k^{n}(H)$ y por lo tanto $\varphi_{n-1}\left(q_{i}\right) \in k^{n-1}(H)$. Por hipótesis de inducción, tenemos que $\varphi_{n-1}\left(q_{i}\right)=q_{i}$, luego, $Q \subseteq \varphi_{n}(Q) \in k^{n}(H)$, pero $Q$ ya era un clan en $k^{n-1}(H)$. Así que $\varphi_{n}(Q)=Q$.

$(3) \Rightarrow(2)$ : Sea $Q=\left\{q_{1}, \ldots, q_{s}\right\} \in k^{n}(G)$. Luego, $q_{i} \in k^{n-1}(G)$ para toda $i=$ $1, \ldots, s$. Por hipótesis de inducción tenemos $\varphi_{n-1}\left(q_{i}\right)=q_{i}$ y así $Q \subseteq \varphi_{n}(Q)$, pero $Q$ ya era un clan de $k^{n-1}(G)$.

De nuevo en el caso de una subgráfica inducida $H$ de $G$ y usando el teorema anterior podemos dar criterios, en términos de cimientos, para saber cuándo un clan iterado de $H$ es un clan iterado de $G$ y viceversa:

Teorema 2.20 Sea $H$ una subgráfica inducida de $G$, sean $Q \in k^{n}(H)$ y $P \in k^{n}(G)$. Entonces: 
1. $\mathcal{B}_{G}(P) \subseteq H$ implica $P \in k^{n}(H)$

2. Si $N_{G}\left[\mathcal{B}_{H}(Q)\right] \subseteq H$ entonces $Q \in k^{n}(G)$

Prueba. El teorema es trivial para el caso $n=1$. Probaremos el paso inductivo.

(1) Sea $P=\left\{p_{1}, \ldots, p_{r}\right\}$ con $p_{i} \in k^{n-1}(G)$. Por hipótesis de inducción, $p_{i} \in$ $k^{n-1}(H)$, así que $P$ es una completa de $k^{n-1}(H)$. Sea $\hat{P} \in k^{n}(H)$ con $P \subseteq \hat{P}$. Sea $\varphi_{0}: H \rightarrow G$ la inclusión y $\varphi_{n}$ cualquier extensión de $\varphi_{0}$ a $k^{n}(H)$. Entonces, usando el teorema anterior, tenemos: $P=\varphi_{n-1}(P) \subseteq \varphi_{n-1}(\hat{P}) \subseteq \varphi_{n}(\hat{P})$. Pero $P$ es un clan de $k^{n-1}(G)$, así que $\varphi_{n}(\hat{P})=P$. Si $q \in \hat{P} \backslash P$, tenemos $\varphi_{n-1}(q)=p_{j} \in k^{n-1}(H)$ para alguna $p_{j} \in P$, pero entonces, por el teorema anterior, $q=\varphi_{n-1}(q)=p_{j} \in P$, contrario al supuesto $q \in \hat{P} \backslash P$. Luego, $\hat{P}=P$ y $P \in k^{n}(H)$.

(2) Sea $Q=\left\{q_{1}, \ldots, q_{r}\right\}$. Por hipótesis de inducción, $q_{i} \in k^{n-1}(G)$ para toda $i$. Sea $\hat{Q} \in k^{n}(G)$ con $Q \subseteq \hat{Q}$. Para llegar a una contradicción, supongamos que $Q \subsetneq \hat{Q}$. Tome cualesquiera $q \in Q$ y $p \in \hat{Q}-Q$. Por el inciso anterior de este teorema, tenemos $\mathcal{B}_{G}(p) \nsubseteq H$. Observamos que:

$$
(*)\left\{\begin{array}{l}
q \in \mathcal{B}_{k^{n-1}(H)}(Q) \cap \mathcal{B}_{k^{n-1}(G)}(\hat{Q}) \\
p \in \mathcal{B}_{k^{n-1}(G)}(\hat{Q}) \\
\mathcal{B}_{G}(p) \nsubseteq H \\
p \text { y } q \text { son adyacentes en } k^{n-1}(G)
\end{array}\right.
$$

Si ahora tomamos cualquier $q^{\prime} \in q \cap p$ y cualquier $p^{\prime} \in p$ tal que $\mathcal{B}_{G}\left(p^{\prime}\right) \nsubseteq H$, tenemos que $p^{\prime}$ y $q^{\prime}$ de nuevo satisfacen $(*)$, si tan sólo cambiamos cada $n-1$ por un $n-2$. Iterando este proceso, llegamos a un par de clanes $\bar{q} \in k(H) \cap k(G)$ y $\bar{p} \in k(G)$ que satisfacen:

$$
\left\{\begin{array}{l}
\bar{q} \in \mathcal{B}_{k(H)}(Q) \cap \mathcal{B}_{k(G)}(\hat{Q}) \\
\bar{p} \in \mathcal{B}_{k(G)}(\hat{Q}) \\
\bar{p} \nsubseteq H \\
\bar{p} \text { y } \bar{q} \text { son adyacentes en } k(G)
\end{array}\right.
$$

Tome ahora $x \in \bar{q} \cap \bar{p}$ y $y \in \bar{p}-H$. Necesariamente, $x$ y $y$ son adyacentes en $G$, pero entonces $y \in N_{G}[x] \subseteq N_{G}\left[\mathcal{B}_{H}(Q)\right] \subseteq H$. Contradicción. 


\section{Gráficas de Johnson}

El estudio del diámetro de las gráficas iteradas de clanes empieza en 1983 cuando Hedman [32] muestra un ejemplo de de una gráfica a la que le crece el diámetro una vez y pregunta si existen gráficas a las que les crezca el diámetro dos veces seguidas y también pregunta si existe alguna gráfica $G$ con $\lim _{n \rightarrow \infty} \operatorname{diam}\left(k^{n}(G)\right)=\infty$. Más tarde el propio Hedman demuestra que a las gráficas de tiempo (no triviales) les decrece el diámetro en 1 bajo cada iteración del operador de clanes [33](1984) y prueba su famoso teorema del diámetro [35](1986) ( ver corolario 2.5).

En el mismo año tanto Balakrishnan y Paulraja [3](1986) como Peyrat, Rall y Slater [59](1986) dan respuesta afirmativa a la primera pregunta de Hedman. Estos últimos dan ejemplos de gráficas a las que les crece el diámetro hasta 4 veces seguidas y conjeturan que para toda $n$ existe una gráfica a la que le crece el diámetro $n$ veces seguidas. También, expresan su creencia de que no existen gráficas de diámetro divergente al iterar el operador de clanes.

Cuatro años más tardó en aparecer el siguiente trabajo sobre diámetros y gráficas de clanes cuando Chen y Lih [10](1990) generalizan el resultado de Hedman sobre gráficas de tiempo a gráficas cordales. Luego Dutton y Brigham [14](1995) usan las técnicas existentes para estudiar el comportamiento del radio de las gráficas bajo el operador de clanes.

En 1999 Larrión y Neumann-Lara [39] dan respuesta afirmativa a la segunda pregunta de Hedman (y negativa a la creencia de Peyrat, Rall y Slater): Sí existen gráficas $G$ con $\lim _{n \rightarrow \infty} \operatorname{diam}\left(k^{n}(G)\right)=\infty$. Sin embargo, a los ejemplos presentados les crece el diámetro como $\left\lfloor\frac{n}{3}\right\rfloor$, de modo que éstos no resuelven la conjetura de Peyrat, Rall y Slater. Esta había sido, de hecho, resuelta un año antes por Bornstein y Szwarcfiter:

Teorema 2.21 (Teorema del diámetro de Bornstein y Szwarcfiter (1998) [9]). Para cada $n$ existe una gráfica $G$ tal que $\operatorname{diam}\left(k^{n}(G)\right)=\operatorname{diam}(G)+n$.

La prueba de Bornstein y Szwarcfiter construye ejemplos de tales gráficas usando una construcción muy especial a la que llaman $H(G, d)$ : Empezando con una gráfica $G$ y un número entero $d$ toman dos copias isomorfas de $G$ y usando trayectorias de longitud $d$ unen cada vértice de cada copia de $G$ a un nuevo vértice, como se muestra 
en la figura 2.4. Luego toman $G$ igual a la $i$-ésima gráfica de líneas de una completa $G=L^{i}\left(K_{m}\right)$ y demuestran que para valores adecuadamente escogidos de $d, i$ y $m$ puede hacerse crecer el diámetro de $H\left(L^{i}\left(K_{m}\right), d\right)$ tantas veces como se desee.

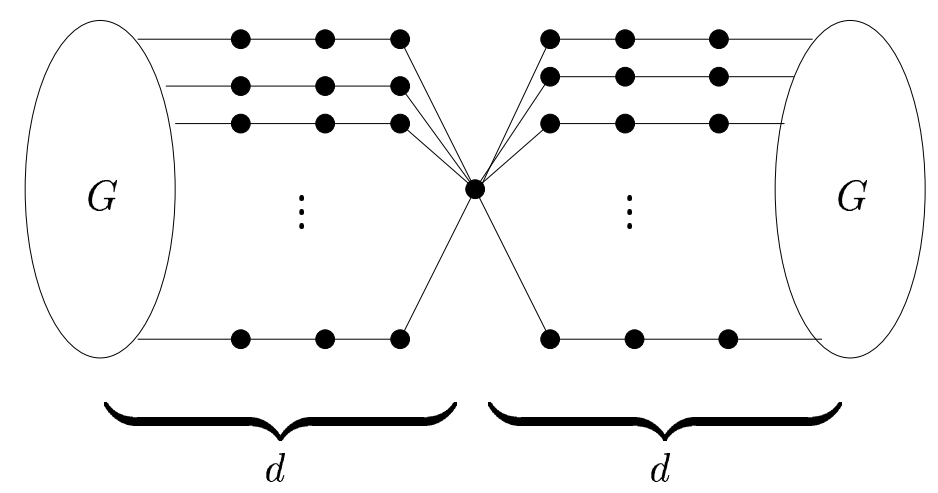

Figura 2.4: La construcción de $H(G, d)$

Por otro lado, como reportamos en [60,62], podemos encontrar ejemplos más naturales de gráficas a las que les crece el diámetro cualquier número de veces seguidas entre las gráficas de Johnson:

Definición 2.22 Dado un conjunto finito $M$ y un entero $0 \leq r \leq|M|$, la gráfica de Johnson $J(M, r)$ es la gráfica que tiene por vértices a todos los subconjuntos de $M$ de orden $r$, dos de ellos son adyacentes si y solamente si comparten exactamente $r-1$ elementos. Si $0 \leq r \leq m$ también escribimos: $J(m, r)=J(\{1, \ldots, m\}, r)$.

Las gráficas de Johnson tienen muchas propiedades de simetría muy interesantes y han sido estudiadas ampliamente. Es bien conocido (y fácil de probar) que dados dos vértices $S_{1}, S_{2} \in J(m, r)$ podemos calcular fácilmente su distancia como $d\left(S_{1}, S_{2}\right)=$ $\left|S_{1}-S_{2}\right|=\left|S_{2}-S_{1}\right|$. En particular, el diámetro de una gráfica de Johnson es

$$
\operatorname{diam}(J(m, r))=\min \{r, m-r\}
$$

Se sigue que cuando $r \in\{0,1, m-1, m\}, J(m, r)$ es una gráfica completa. De modo que, desde el punto de vista de las gráficas de clanes, las gráficas de Johnson interesantes son aquéllas en las que $2 \leq r \leq m-2$. Otra propiedad interesante es que $J(m, r) \cong J(m, m-r)$.

Una propiedad interesante de las gráficas de Johnson en relación a las gráficas iteradas de clanes es que para $2 \leq r \leq m-2$ tanto $J(m, r-1)$ como $J(m, r+1)$ son subgráficas 
inducidas de $k(J(m, r))$. Estas subgráficas inducidas dan una partición de $k(J(m, r))$ en dos clases, es decir, todo vértice de $k(J(m, r))$ pertenece a exactamente una de estas dos subgráficas inducidas. Este bonito resultado es fácil de probar, pero omitimos su prueba porque no necesitaremos este resultado.

Sin embargo, una generalización parcial de ese resultado vale para las gráficas iteradas de clanes y nos será de mucha utilidad: Con frecuencia $J(m, r+n)$ es una subgráfica inducida de $k^{n}(J(m, r))$ :

Teorema 2.23 Sean $m, r \in \mathbb{N}$ con $2 \leq r \leq m-1$. Entonces para cada $n \in \mathbb{N}$ tal que $1 \leq n \leq \min \{r-1, m-r\}$ hay un morfismo (necesariamente inyectivo)

$$
\varphi_{n}: J(m, r+n) \rightarrow k^{n}(J(m, r))
$$

tal que para cada $S \in V(J(m, r+n))$,

$$
\mathcal{B}\left(\varphi_{n}(S)\right)=J(S, r)
$$

Prueba. Supongamos $n=1$. Tome $M=\{1, \ldots, m\}$ y $S \in V(J(M, r+1))$. Observamos que el conjunto $J(S, r)$ induce una subgráfica completa de $J(M, r)$ : Como para cada $R_{1}, R_{2} \in J(S, r)$ con $R_{1} \neq R_{2}$, tenemos que $\left|R_{1} \cap R_{2}\right|=r-1$, se sigue que $R_{1}$ y $R_{2}$ son adyacentes en $J(M, r)$.

En este caso, $J(S, r)$ ya es un clan de $J(M, r)$ : Si $R \in J(M, r)-J(S, r)$, entonces $|S-R| \geq 2$ y, como $r \geq 2$, siempre hay un vértice $R^{\prime}$ de $J(M, r)$ en $J(S, r)$ tal que $\left|R^{\prime}-R\right| \geq 2$.

Ahora, la función $\varphi_{1}: J(M, r+1) \rightarrow k(J(M, r))$ dada por $\varphi_{1}(S)=J(S, r)$ es un morfismo porque si $S, S^{\prime} \in J(M, r+1)$ son adyacentes, entonces $\left|S \cap S^{\prime}\right|=r$ y así $J(S, r) \cap J\left(S^{\prime}, r\right) \supseteq\left\{S \cap S^{\prime}\right\} \neq \varnothing$. Claramente, $\mathcal{B}(J(S, r))=J(S, r)$.

Ahora supongamos que $n \geq 2$. Por hipótesis de inducción, tenemos un morfismo

$$
\varphi_{n-1}: J(M, r+n-1) \rightarrow k^{n-1}(J(M, r))
$$

tal que $\mathcal{B}\left(\varphi_{n-1}(T)\right)=J(T, r)$ para toda $T \in V(J(M, r+n-1))$.

Como antes, para cada $S \in J(M, r+n)$ el conjunto $J(S, r+n-1)$ induce una subgráfica completa de $J(M, r+n-1)$, así que $\varphi_{n-1}(J(S, r+n-1))$ induce una subgráfica completa de $k^{n-1}(J(M, r))$. Definamos $C_{S}=\varphi_{n-1}(J(S, r+n-1))$. 
Para cada $S \in J(M, r+n)$, seleccionemos un clan fijo $Q_{S}$ de $k^{n-1}(J(M, r)$ ) (un vértice en $\left.k^{n}(J(M, r))\right)$ que contenga a $C_{S}$. Entonces

$$
\begin{aligned}
\mathcal{B}\left(Q_{S}\right) & =\bigcup_{q \in Q_{S}} \mathcal{B}(q) \supseteq \bigcup_{q \in C_{S}} \mathcal{B}(q)=\bigcup_{T \in J(S, r+n-1)} \mathcal{B}\left(\varphi_{n-1}(T)\right) \\
& =\bigcup_{T \in J(S, r+n-1)} J(T, r)=J(S, r) .
\end{aligned}
$$

Pero si $R \in J(M, r)-J(S, r)$, entonces $|S-R| \geq n+1$ y así, como $r \geq n+1$, hay una $R^{\prime} \in J(S, r)$ tal que $d\left(R, R^{\prime}\right) \geq n+1$. Ahora, el teorema 2.17 le prohíbe a $R$ la pertenencia a $\mathcal{B}\left(Q_{S}\right)$ porque $\max D\left(\mathcal{B}\left(Q_{S}\right), \mathcal{B}\left(Q_{S}\right)\right) \leq n$. Entonces tenemos $\mathcal{B}\left(Q_{S}\right)=J(S, r)$.

Finalmente, si $S, S^{\prime} \in J(M, r+n)$ y $\left|S \cap S^{\prime}\right|=r+n-1$, entonces $Q_{S} \cap Q_{S^{\prime}} \supseteq$ $\left\{\varphi_{n-1}\left(S \cap S^{\prime}\right)\right\} \neq \varnothing$.

Así, el morfismo que se requiere está dado por: $\varphi_{n}(S)=Q_{S}$

Ahora podemos dar una segunda prueba al teorema del diámetro de Bornstein y Szwarcfiter (teorema 2.21):

Teorema 2.24 Sean $r \geq 2, m \geq 4 r-2$ y $G=J(m, r)$, entonces para cada $n$ con $1 \leq n \leq r-1: \operatorname{diam}\left(k^{n}(G)\right)=\operatorname{diam}(G)+n$.

Prueba. Sean $S_{1}=\{1, \ldots, r+n\}, S_{2}=\{r+n+1, \ldots, 2 r+2 n\}$ y $M=$ $\{1, \ldots, m\}$. Por el teorema 2.23, hay un morfismo $\varphi: J(M, r+n) \rightarrow k^{n}(J(M, r))$ tal que $\mathcal{B}\left(\varphi\left(S_{1}\right)\right)=J\left(S_{1}, r\right)$ y $\mathcal{B}\left(\varphi\left(S_{2}\right)\right)=J\left(S_{2}, r\right)$. Como $J\left(S_{1}, r\right) \cap J\left(S_{2}, r\right)=\varnothing$ y $\min D_{G}\left(J\left(S_{1}, r\right), J\left(S_{2}, r\right)\right)=r$, el teorema 2.17 nos dice que $d\left(\varphi\left(S_{2}\right), \varphi\left(S_{2}\right)\right)=$ $r+n$. Entonces $\operatorname{diam}\left(k^{n}(G)\right) \geq r+n$, pero $\operatorname{diam}(G)=r$ y ya sabíamos que para cualquier gráfica, $\operatorname{diam}\left(k^{n}(G)\right) \leq \operatorname{diam}(G)+n$.

Varios problemas abiertos emergen en esta dirección, por ejemplo existe cierta evidencia experimental que indica que a las gráficas de Johnson les crece el diámetro más allá de los límites que hemos probado:

Problema 1 ¿Es cierto que $\operatorname{diam}\left(k^{n}(J(m, r))\right)=r+n$ si $2 \leq r$ y $r+n \leq\left\lfloor\frac{m}{2}\right\rfloor$ ? 
Cabe mencionar que toda gráfica de Johnson $J(m, r)$ con $2 \leq r \leq m-2$ es clan divergente: El octaedro es un retracto de todas estas gráficas. Esto implica que al igual que con el octaedro, el orden de las gráficas de Johnson crece superexponencialmente al aplicar repetidamente el operador de clanes y deja poco espacio para la experimentación computacional en busca de una respuesta negativa al problema anterior.

Por otro lado, en el caso de que el problema 1 tenga una respuesta afirmativa ¿valdría también para gráficas de Johnson infinitas?

Problema 2 ¿Es cierto que $\operatorname{diam}\left(k^{n}\left(J\left(\aleph_{0}, r\right)\right)\right)=r+n$ para toda $r \geq 2$ y $n \geq 0$ ?

De manera más general, podemos hacernos la siguiente pregunta:

Problema 3 ¿Existe alguna gráfica $G$ (no necesariamente finita) de diámetro finito tal que $\operatorname{diam}\left(k^{n}(G)\right)=\operatorname{diam}(G)+n$ para toda $n$ ?

Observamos que los teoremas 1.1 y 1.2 valen también para productos fuertes numerables, de modo que si problema 1 tiene una respuesta afirmativa, la gráfica

$$
J(6,2) \otimes J(7,2) \otimes \cdots \otimes J(m, 2) \otimes \cdots
$$

nos daría una respuesta afirmativa al problema anterior. No podemos hacer cosa parecida usando solamente el teorema 2.24 .

Por supuesto, todo el trabajo hecho a la fecha sobre gráficas iteradas de clanes es sobre gráficas finitas, de modo que es natural preguntar:

Problema 4 (Problema del diámetro)

¿Existe alguna gráfica finita $G$ tal que $\operatorname{diam}\left(k^{n}(G)\right)=\operatorname{diam}(G)+n$ para toda $n$ ?

Este último problema es el paso obvio siguiente después del teorema del diámetro de Bornstein y Szwarcfiter. Dedicaremos la siguiente sección a consideraciones generales sobre cómo debería ser una gráfica que resolviera afirmativamente el problema anterior. 


\section{Diámetros}

El resultado principal de esta sección es el teorema 2.28, que nos presenta 5 formas equivalentes del problema 4 . Pero necesitaremos algunos lemas:

Lema 2.25 Sea $G$ una gráfica de diámetro $d \geq 1$ y sean $x, y$ dos vértices de $G$. Entonces $\min \mathcal{D}(N[x],\{y\}) \leq d-1$.

Prueba. ¿Dónde cae el segundo vértice de una trayectoria de longitud mínima entre $x$ y $y$ ?

Teorema 2.26 Sean $p \in k^{n}(G)$ y $q \in k^{n-1}(G)$ con $q \in p$. Entonces: $p \in q^{*}$. En particular, $q^{*} \in p^{*}$ y si $x \in \mathcal{B}_{m}(p)$ tenemos que $x^{*} \in \mathcal{B}_{m+2}\left(p^{*}\right)$ para $0 \leq m<n$.

Prueba. Inmediato.

Teorema 2.27 Si $x \in G$, entonces $N_{G}[x] \subseteq \mathcal{B}_{G}\left(x^{*}\right)$. Si $q \in k^{n}(G)$ entonces $N_{G}\left[\mathcal{B}_{G}(q)\right] \subseteq \mathcal{B}_{G}\left(q^{*}\right)$

Prueba. Si $y \in N_{G}[x]$, hay un clan $Q$ de $G$ que contiene a la arista $\{x, y\}$. Entonces, por definición $Q \in x^{*}$ y $y \in \mathcal{B}_{G}\left(x^{*}\right)$. Por el teorema anterior, si $x \in \mathcal{B}_{0}(q)$, tenemos que $x^{*} \in \mathcal{B}_{2}\left(q^{*}\right)$, pero $N_{G}[x] \subseteq \mathcal{B}_{0}\left(x^{*}\right) \subseteq \mathcal{B}_{G}\left(q^{*}\right)$

Diremos que $Q \in k^{n}(G)$ cubre a $x \in k^{m}(G)$ si $x \in \mathcal{B}_{m}(Q)$ y que $Q$ cubre a $k^{m}(G)$ si $\mathcal{B}_{m}(Q)=V\left(k^{m}(G)\right)$. Diremos que una corbata iterada $Q \in k^{n+2}(G)$ es una corbata pura, si cada $q \in \mathcal{B}_{m}(Q)$ es una corbata para $m=2,3, \ldots, n+2$.

Tenemos ahora el teorema principal de la sección:

Teorema 2.28 Son equivalentes:

1. Existe una gráfica $G_{1}$ tal que $\operatorname{diam}\left(k^{n}\left(G_{1}\right)\right)=\operatorname{diam}\left(G_{1}\right)+n$ para toda $n$.

2. Existe una gráfica $G_{2}$ con infinitas corbatas puras. 
3. Existe una gráfica $G_{3}$ con infinitos clanes iterados $Q$ con la propiedad: $S i$ $Q \in k^{n}\left(G_{3}\right)$ entonces $\mathcal{B}_{m}(Q) \subsetneq k^{m}\left(G_{3}\right)$ para toda $m$ con $0 \leq m<n$.

4. Existe una gráfica $G_{4}$ tal que hay infinitos clanes iterados $Q$ con la propiedad: $\mathcal{B}_{0}(Q) \subsetneq G_{4}$.

5. Existe una gráfica $G_{5}$ tal que $\operatorname{diam}\left(k^{n}\left(G_{5}\right)\right) \geq n$ para toda $n$.

\section{Prueba.}

$(\mathbf{1}) \Rightarrow(\mathbf{2})$ : Tomemos $G_{2}=G_{1}$. Sean $P, Q \in k^{n}(G)$ tal que $d(P, Q)=\operatorname{diam}\left(G_{2}\right)+n$. Como $\operatorname{diam}\left(G_{2}\right) \geq 2$, tenemos, por la fórmula de la distancia, que $\mathcal{B}_{0}(P) \cap$ $\mathcal{B}_{0}(Q)=\varnothing$. Luego, $d(P, Q)=\min \mathcal{D}(\mathcal{B}(P), \mathcal{B}(Q))+n$ también por la fórmula de la distancia. Si alguno de entre $P$ y $Q$ no es una corbata pura, digamos $v^{*} \in \mathcal{B}_{m}(P)$, por el teorema 2.27, tenemos que $N[x] \subseteq \mathcal{B}_{0}\left(v^{*}\right) \subseteq \mathcal{B}_{0}(P)$, para cualquier $x \in \mathcal{B}_{0}(v)$. Entonces, usando el lema 2.25:

$$
\begin{aligned}
d(P, Q) & =\min \mathcal{D}(\mathcal{B}(P), \mathcal{B}(Q))+n \\
& \leq \min \mathcal{D}(N[x], \mathcal{B}(Q))+n \\
& \leq \operatorname{diam}\left(G_{2}\right)-1+n \\
& <\operatorname{diam}\left(G_{2}\right)+n
\end{aligned}
$$

Que es una contradicción. Entonces, en cada nivel $k^{n}\left(G_{2}\right), G_{2}$ tiene al menos dos corbatas puras.

$(2) \Rightarrow(3)$ : Tome $G_{3}=k^{2}\left(G_{2}\right)$ y $x \in k^{m}\left(G_{2}\right)$, con $m \geq 0$. Luego $x^{*} \in k^{m+2}\left(G_{2}\right)=$ $k^{m}\left(G_{3}\right)$. Ninguna corbata pura de $G_{2}$ cubre a $x^{*}$.

$(3) \Rightarrow(4)$ : Obvio.

$(4) \Rightarrow(5)$ : Como $G_{4}$ es finita, hay algún vértice $x \in G_{4}$ tal que hay un número infinito de clanes iterados que no lo cubren. Si $P \in k^{n}\left(G_{4}\right)$ no cubre a $x, p$ tampoco cubre a $x$ para cualquier $p \in \mathcal{B}_{m}(P)$ con $1 \leq m<n$. Sea $\left\{P_{1}, P_{2}, \ldots\right\}$ una familia de clanes iterados de $G_{4}$ con $P_{i} \in k^{i}\left(G_{4}\right)$ y $x \notin \mathcal{B}_{0}\left(P_{i}\right)$. Sea $G_{4}^{\prime}$ una copia disjunta de $G_{4}$ y $x^{\prime}$ el vértice correspondiente a $x$ en $G_{4}^{\prime}$. Sean $\left\{P_{1}^{\prime}, P_{2}^{\prime}, \ldots\right\}$ los clanes iterados de $G_{4}^{\prime}$ que corresponden a los de $G_{4}$. Podemos ahora tomar a $G_{5}$ como las suma punteada de $G_{4}$ y $G_{4}^{\prime}$ con respecto a $x$ y $x^{\prime}$, es decir: $G_{5}=\left(G_{4}, x\right) \cdot\left(G_{4}^{\prime}, x^{\prime}\right)$. Observamos que $G_{4}$ y $G_{4}^{\prime}$ son subgráficas 
inducidas de $G_{5}$ y por construcción, tenemos que $N_{G_{5}}\left[\mathcal{B}_{G_{4}}\left(P_{i}\right)\right] \subseteq G_{4}$ para toda $i$. Como lo mismo ocurre con todas las $P_{i}^{\prime}$, tenemos que, por el teorema 2.20(2), tanto $P_{i}$ como $P_{i}^{\prime}$ son clanes iterados de $G_{5}$. Luego

$$
\operatorname{diam}\left(k^{n}\left(G_{5}\right)\right) \geq \min \mathcal{D}\left(\mathcal{B}\left(P_{n}\right), \mathcal{B}\left(P_{n}^{\prime}\right)\right)+n \geq n+2 \geq n
$$

$(5) \Rightarrow(1)$ : Sea $d=\operatorname{diam}\left(G_{5}\right)$. Por el teorema de Hedman (corolario 2.5), $G_{5}$ solamente puede no incrementar su diámetro en un máximo de $d$ aplicaciones del operador de clanes. De lo contrario, para una $n$ suficientemente grande, tendríamos:

$$
\operatorname{diam}\left(k^{n}\left(G_{5}\right)\right) \leq \operatorname{diam}\left(G_{5}\right)+n-(d+1)<n
$$

En particular, $G_{5}$ puede no incrementar su diámetro solamente en un número finito de ocasiones. Tomemos ahora

$$
m=\max \left(\{-1\} \cup\left\{m \in \mathbb{N}: \operatorname{diam}\left(k^{m+1}\left(G_{5}\right)\right) \leq \operatorname{diam}\left(k^{m}(G)\right)\right\}\right)
$$

Ahora sólo basta tomar $G_{1}=k^{m+1}\left(G_{5}\right)$.

Sea $b(G, n)=\min \left\{\left|\mathcal{B}_{0}(Q)\right|: Q \in k^{n}(G)\right\}$. Es claro que para toda $G, b(G, n)$ es monótona creciente en $n$. El teorema anterior nos dice que existe una gráfica $G_{1}$ a la que siempre le crece el diámetro ssi existe una gráfica $G_{4}$ tal que $b\left(G_{4}, n\right)<$ $\left|G_{4}\right|$ para toda $n$. Una manera en que a uno se le ocurriría que se puede dar una respuesta negativa al problema 4 es tratando de demostrar que para cualquier gráfica $G, b(G, n)<b(G, n+1)$ siempre que $b(G, n)<|G|$. Sin embargo esto es falso: incluso en ejemplos tan sencillos como la suma punteada de dos cuadrados $G=\mathcal{C}_{4} \cdot \mathcal{C}_{4}$ se tiene $b(G, 3)=b(G, 4)$ y $b(G, 5)=b(G, 6)$ :

\begin{tabular}{|c||c|c|c|c|c|c|c|}
\hline$n=$ & 1 & 2 & 3 & 4 & 5 & 6 & 7 \\
\hline$b(G, n)=$ & 2 & 3 & 4 & 4 & 6 & 6 & $|G|=7$ \\
\hline
\end{tabular}

Quizá algún otro enfoque semejante podría dar mejores frutos. 


\section{Capítulo 3}

\section{Relojes}

Las gráficas de relojería o simplemente los relojes, fueron introducidos por Larrión y Neumann-Lara en [41] y ya han probado ser extremadamente útiles para construir ejemplos y contraejemplos en el estudio de las gráficas iteradas de clanes. Por ejemplo, han sido usadas para construir ejemplos de: Gráficas clan divergentes primitivas (de crecimiento lineal) [41], gráficas con cualquier periodo [16,17], gráficas clan divergentes $r$-coafines saturadas [61] y gráficas de comparabilidad de órdenes parciales con la propiedad del punto fijo que sean clan divergentes $[43,46]$. Precursores de los relojes pueden ser encontrados en [38] e incluso en [15](1973) en donde también se usaron para construir ejemplos.

La versión de relojes que presentamos aquí es ligeramente más general que la presentada por Larrión y Neumann-Lara en [41]. La mayoría de los resultados en las primeras tres secciones son adecuaciones de los que se presentan en $[41,43]$ a este enfoque más general. En la cuarta sección veremos las similitudes y diferencias de ambas versiones de relojes. Aunque la presentación que se da aquí es autocontenida recomendamos ampliamente al lector la revisión de los trabajos [41] y [43].

Los resultados principales de este capítulo son los teoremas 3.5 y 3.10. Pero también construiremos ejemplos que serán indispensables para el siguiente capítulo. 


\section{Gráficas cíclicamente segmentadas}

Definición 3.1 Sea $G$ una gráfica. Una segmentación cíclica de $G$ es un conjunto de $s \geq 3$ segmentos $\mathcal{S}=\left\{G_{0}, G_{1}, \ldots, G_{s-1}\right\}$ que satisface las siguientes propiedades:

1. $\mathcal{S}$ es una partición de $V(G)$.

2. Cada $G_{i}$ induce una subgráfica completa en $G$.

3. Toda arista de $G$ está en la unión de a lo más dos segmentos consecutivos módulo s

4. Todo triángulo de $G$ está en la unión de a lo más dos segmentos consecutivos módulo $s$

En este caso decimos que $G$ admite una segmentación de $s$ segmentos. Diremos que $G_{j}$ es el segmento siguiente de $G_{i}$ y que $G_{i}$ es el segmento anterior de $G_{j}$ siempre que $j \equiv i+1 \bmod s$. En adelante, entenderemos que toda referencia a los índices de los segmentos se toma módulo $s$, por ejemplo, que $G_{s}=G_{0}$. Una gráfica cíclicamente segmentada es una gráfica $G$ provista de una segmentación cíclica $\mathcal{S}$. Si $G$ es una gráfica cíclicamente segmentada con segmentación $\mathcal{S}=\left\{G_{0}, G_{1}, \ldots, G_{s-1}\right\}$ y $x \in G_{i}$, denotamos por $F_{x}$ al conjunto de vecinos de $x$ en $G_{i+1}$ y por $B_{x}$ al conjunto de vecinos de $x$ en $G_{i-1}$. También, si $A \subseteq G_{i}$, definimos $F A=\bigcap_{a \in A} F_{a}$ y $B A=\bigcap_{a \in A} B_{a}$.

\section{Los relojes y sus clanes}

Definición 3.2 Sea $G$ una gráfica cíclicamente segmentada con segmentación $\mathcal{S}=$ $\left\{G_{0}, G_{1}, \ldots, G_{s-1}\right\}$. Decimos que $G$ es un reloj si satisface:

(I) Para toda $G_{i} \in \mathcal{S}$ y para toda $x \in G_{i}$ :

$$
F_{x} \neq \varnothing
$$

(II) Para toda $G_{i} \in \mathcal{S}$ y para cualesquiera $x, y \in G_{i}$ existe una $z \in G_{i}$ tal que:

$$
F_{x} \cap F_{y}=\varnothing \Rightarrow F_{z} \supseteq F_{x} \cup F_{y}
$$


(III) Para toda $G_{i} \in \mathcal{S}$ y para cualesquiera $x, y \in G_{i}$ existe una $z \in G_{i}$ tal que:

$$
F_{x} \cap F_{y} \neq \varnothing \Rightarrow F_{z}=F_{x} \cap F_{y} \text { y } B_{z} \supseteq B_{x} \cup B_{y}
$$

Los relojes así definidos tienen múltiples propiedades. Analizaremos algunas a continuación.

Sea $G$ un reloj con segmentación $\mathcal{S}=\left\{G_{0}, G_{1}, \ldots, G_{s-1}\right\}$

Observamos que todo clan de $G$ está contenido en la unión de a lo más 2 segmentos consecutivos. Supongamos que $Q \subseteq G_{i} \cup G_{i+1}$ es un clan de $G$ y que $Q \cap G_{i} \neq \varnothing \neq$ $Q \cap G_{i+1}$. Sea $X=Q \cap G_{i}$ y $Y=Q \cap G_{i+1}$. Entonces, notamos que $X=B Y$ y $Y=F X$, pues de otro modo $Q$ no sería clan. Como $Y \neq \varnothing$, por la propiedad (III) tenemos que $F X=\bigcap_{x \in X} F_{x}=F_{z}$ para alguna $z \in G_{i}$. Luego $Q=F_{z} \cup B F_{z}$. Notemos que si $x \in V(G), F_{x} \cup B F_{x}$ siempre es un clan, sin embargo es posible que $F_{x} \cup B F_{x}=F_{y} \cup B F_{y}$ para $x, y \in V(G), x \neq y$. Por otro lado, si $Q$ es un clan que está contenido en un sólo segmento $G_{i}$, como $G_{i}$ es una completa, se sigue que $Q=G_{i}$. El segmento $G_{i}$ es un clan exactamente cuando $F G_{i}=B G_{i}=\varnothing$. Los dos tipos de clanes descritos cubren todas las posibilidades para clanes de $G$. Para cada $x \in V(G)$ definimos $x^{+}=F_{x} \cup B F_{x}$ y llamamos a estos clanes vértices viejos de $k(G)$ porque como veremos más adelante, estos vértices de $k(G)$ reproducen mucho de la estructura original de $G$. A los otros posibles clanes del tipo $G_{i}$, les llamaremos vértices nuevos de $k(G)$ siempre que estén presentes. También es conveniente llamar segmento bueno ${ }^{1}$ a un segmento $G_{i}$ siempre que $F G_{i+1}=B G_{i+1}=\varnothing$, en caso contrario decimos que es un segmento malo. Note que $x \mapsto x^{+}$es una función de $V(G)$ en $V(k(G))$. Entonces es claro que significa $A^{+}$para cualquier subconjunto $A$ de vértices de $G$. Resumimos nuestras observaciones en el siguiente enunciado:

1. Si $G$ es un reloj con segmentación $\mathcal{S}, x^{+}=F_{x} \cup B F_{x}$ es un clan de $G$ para toda $x \in V(G) ; G_{i} \in \mathcal{S}$ es un clan de $G$ si y sólo si $F G_{i}=B G_{i}=\varnothing$. Todo clan de $G$ es de una de estas dos formas.

\footnotetext{
${ }^{1}$ Tal y como está definida aquí la bondad, parece más natural llamar segmento bueno a $G_{i+1}$ en lugar de a $G_{i}$, la razón por la que hacemos esto es por compatibilidad con los trabajos previos publicados sobre el tema. En los relojes que se presentan en $[16,17,41,43,46,61]$, siempre se satisface $F G_{i}=\varnothing$ para toda $i$ y la condición $B G_{i+1}=\varnothing$ es equivalente a que $F_{x} \neq G_{i+1}$ para toda $x \in G_{i}$, esta última condición es la que se usa para definir la bondad en la literatura y en este contexto es igualmente adecuado llamar bueno a $G_{i}$ o a $G_{i+1}$.
} 
Definimos a continuación $\widetilde{\mathcal{S}}=\left\{\tilde{G}_{0}, \tilde{G}_{1}, \ldots, \tilde{G}_{s-1}\right\}$ con $\tilde{G}_{i}=\left\{x^{+}: x \in G_{i}\right\} \cup\left\{G_{i+1}\right\}$ si $G_{i+1}$ es un clan de $G$ (i.e. si $F G_{i+1}=B G_{i+1}=\varnothing$ ). En caso contrario definimos $\tilde{G}_{i}=\left\{x^{+}: x \in G_{i}\right\}$. Afirmamos que $\widetilde{\mathcal{S}}$ es una segmentación de $k(G)$. En primer lugar, es claro que $\widetilde{\mathcal{S}}$ es una partición de los vértices de $k(G)$ (note que ninguna $\tilde{G}_{i}$ puede ser vacía). Veamos que toda $\tilde{G}_{i}$ es una completa en $k(G)$ :

Si $G_{i+1}, x^{+} \in \tilde{G}_{i}$ tenemos $x^{+} \cap G_{i+1}=F_{x} \cup B F_{x} \cap G_{i+1}=F_{x} \neq \varnothing$, luego cualquier vértice nuevo de un segmento es adyacente en $k(G)$ a todos los demás vértices de su segmento. Si $x^{+}, y^{+} \in \tilde{G}_{i}$ tenemos que o bien $F_{x} \cap F_{y} \neq \varnothing$ (en cuyo caso, $\left.x^{+} \cap y^{+} \neq \varnothing\right)$ o bien $F_{x} \cap F_{y}=\varnothing$, y entonces, por la propiedad (II), tenemos que existe una $z \in G_{i}$ tal que $F_{z} \supseteq F_{x} \cup F_{y}$ esto implica que $z \in B F_{x} \cap B F_{y}$ de lo que concluimos que $x^{+} \cap y^{+} \neq \varnothing$. Se sigue que todo par de vértices viejos en un mismo segmento son adyacentes. Hemos probado pues que toda $\tilde{G}_{i}$ es una completa en $k(G)$.

Si $x^{+}$y $y^{+}$no están en segmentos consecutivos ni iguales no pueden ser adyacentes: si $x^{+} \in \tilde{G}_{i}$ y $y^{+} \in \tilde{G}_{j}$ tenemos que $x \in G_{i}$ y $y \in G_{j}$, luego $x^{+} \subseteq G_{i} \cup G_{i+1}$ y $y^{+} \subseteq G_{j} \cup G_{j+1}$ luego $x^{+} \cap y^{+} \subseteq\left(G_{i} \cup G_{i+1}\right) \cap\left(G_{j} \cup G_{j+1}\right)=\varnothing$. También observamos que dos vértices nuevos nunca son adyacentes entre sí y que un vértice nuevo $G_{i+1} \in \tilde{G}_{i}$ sólo puede ser adyacente a vértices viejos de los segmentos $\tilde{G}_{i} \mathrm{y}$ $\tilde{G}_{i+1}$ (de hecho todo vértice nuevo es adyacente exactamente a todo vértice viejo en su segmento y el siguiente). Hemos probado que toda arista de $k(G)$ está contenida en la unión de a lo más dos segmentos consecutivos de $k(G)$.

Finalmente vemos que no puede haber triángulos que no estén contenidos en dos segmentos consecutivos. Suponga que $Q_{0}, Q_{1}, Q_{2}$ son tres clanes de $G$ que se intersectan mutuamente y que no están en la unión de dos segmentos de $k(G)$. Entonces, necesariamente, la segmentación de $G$ consta de exactamente tres segmentos y sin pérdida de generalidad $Q_{0} \in \tilde{G}_{0}, Q_{1} \in \tilde{G}_{1}, Q_{2} \in \tilde{G}_{2}$, con $\widetilde{\mathcal{S}}=\left\{\tilde{G}_{0}, \tilde{G}_{1}, \tilde{G}_{2}\right\}$. Luego, $Q_{0} \subseteq G_{0} \cup G_{1}, Q_{1} \subseteq G_{1} \cup G_{2}$ y $Q_{2} \subseteq G_{2} \cup G_{0}$. Se sigue que $Q_{0} \cap Q_{1} \subseteq G_{1}$, $Q_{1} \cap Q_{2} \subseteq G_{2}$ y $Q_{2} \cap Q_{0} \subseteq G_{0}$. Como estos tres clanes se intersectan dos a dos, basta con tomar $x \in Q_{0} \cap Q_{1}, y \in Q_{1} \cap Q_{2}$ y $z \in Q_{2} \cap Q_{0}$ para exhibir un triángulo $\{x, y, z\}$ de $G$ que no está en la unión de dos segmentos consecutivos de $G$, contradiciendo la hipótesis de que $G$ es un reloj. Así pues hemos probado que:

2. Si $G$ es un reloj con segmentación $\mathcal{S}=\left\{G_{0}, G_{1}, \ldots, G_{s-1}\right\}, k(G)$ admite la segmentación cíclica: $\widetilde{\mathcal{S}}=\left\{\tilde{G}_{0}, \tilde{G}_{1}, \ldots, \tilde{G}_{s-1}\right\}$, donde $\tilde{G}_{i}=$ $\left\{x^{+}: x \in G_{i}\right\} \cup\left\{G_{i+1}\right\}\left(\right.$ si $\left.F G_{i+1}=B G_{i+1}=\varnothing\right)$ o $\tilde{G}_{i}=\left\{x^{+}: x \in G_{i}\right\}$ (en 


\section{caso contrario).}

Una vez probado que $k(G)$ es una gráfica cíclicamente segmentada, tenemos derecho a usar $F$ y $B$ para describir las adyacencias de los vértices de $k(G)$ de la misma forma que en cualquier otra gráfica segmentada. Note, sin embargo, que $G_{i}$ puede ser tanto un conjunto de vértices de $G$, como un vértice de $k(G)$ y en consecuencia $F_{G_{i}}$ es un conjunto de vértices de $k(G)$, pero $F G_{i}$ es un conjunto de vértices de $G$.

Notamos de inmediato que:

3. $F_{G_{i}}=\left(G_{i}\right)^{+}, B_{G_{i}}=\varnothing$ para toda i.

Ahora notamos que por las propiedades (I) y (III), si $x \in G_{i}$ y $X=\left\{y \in G_{i}: F_{x}=F_{y}\right\}$ entonces necesariamente existe una $\bar{x} \in X$ tal que $\left(F_{\bar{x}}=F_{x}\right.$ y) $B_{\bar{x}} \supseteq B_{y}$ para toda $y \in X$. Este vértice puede no ser único, pero para cada $x \in V(G)$ podemos escoger uno de tales vértices y así obtener una función de $V(G)$ en $V(G)(x \mapsto \bar{x})$ con las propiedades:

4. Si $x \in G_{i}$, entonces $\bar{x} \in G_{i}, F_{x}=F_{\bar{x}}, F_{y}=F_{x} \Rightarrow B_{\bar{x}} \supseteq B_{y} \mathbf{y} F_{y}=F_{x} \Rightarrow$ $\bar{x}=\bar{y}$ para toda $y \in G_{i}$.

Por la propiedad (III), tenemos que si $F_{x} \subseteq F_{y}$ entonces existe $z$ tal que $F_{z}=$ $F_{x} \cap F_{y}=F_{x}$ y $B_{z} \supseteq B_{x} \cup B_{y}$, pero por las propiedades de $\bar{x}$, tenemos que $B_{\bar{x}} \supseteq$ $B_{z} \supseteq B_{x} \cup B_{y}$. También $\alpha \in F_{x}$ si y sólo si $x \in B_{\alpha}$, pero $B_{\alpha} \subseteq B_{\bar{\alpha}}$ y por lo tanto $\alpha \in F_{x} \Rightarrow \bar{\alpha} \in F_{x}$. Como $F_{x}=F_{\bar{x}}$ para cualquier $x$, tenemos que $x \in B_{\alpha}$ si y sólo si $\bar{x} \in B_{\alpha}$. Así:

5. $x \in B_{\alpha}$ si y sólo si $\bar{x} \in B_{\alpha} ; \alpha \in F_{x}$ implica $\bar{\alpha} \in F_{x}$ y $F_{x} \subseteq F_{y}$ implica $B_{y} \subseteq B_{\bar{x}}$.

También es inmediato lo siguiente:

6. $x^{+}=y^{+} \Leftrightarrow F_{x}=F_{y} \Leftrightarrow \bar{x}=\bar{y}$; Como $F_{x}=F_{\bar{x}}$ tenemos $\bar{x}=\bar{x} \mathbf{y} \bar{x}^{+}=x^{+}$. 
Si $\alpha^{+} \in F_{x^{+}}$, se sigue que existe $\beta \in B F_{\alpha} \cap F_{x}=\alpha^{+} \cap x^{+}$. Entonces $F_{\alpha} \subseteq F_{\beta}$ y $x \in B_{\beta}$, pero $x \in B_{\beta} \subseteq B_{\bar{\alpha}}$ y así $\bar{\alpha} \in F_{x}$ (prop. (III)). Por otro lado, si $\bar{\alpha} \in F_{x}$, entonces, claramente $\bar{\alpha} \in B F_{\bar{\alpha}} \cap F_{x}=\bar{\alpha}^{+} \cap x^{+}$, luego $\bar{\alpha}^{+}$y $x^{+}$son adyacentes en $k(G)$ y $\alpha^{+}=\bar{\alpha}^{+} \in F_{x^{+}}$. Entonces:

7. $\alpha^{+} \in F_{x^{+}}$si y sólo si $\bar{\alpha} \in F_{x}$.

Si $\bar{\alpha} \in F_{x}$ es claro que $\alpha^{+}=\bar{\alpha}^{+} \in\left(F_{x}\right)^{+}$. Por otro lado, si $\alpha^{+} \in\left(F_{x}\right)^{+}$, tenemos que existe $\beta \in F_{x}$ tal que $\alpha^{+}=\beta^{+}$, pero entonces $\bar{\beta} \in F_{x}$ y $\bar{\alpha}=\bar{\beta}$ y así:

8. $\alpha^{+} \in\left(F_{x}\right)^{+}$si y sólo si $\bar{\alpha} \in F_{x}$.

Como $B_{G_{i}}=\varnothing$ para toda $i$, tenemos que $G_{i} \notin F_{x^{+}}$para cualquier $x \in V(G)$. Usando los dos incisos anteriores, tenemos:

9. $F_{x^{+}}=\left(F_{x}\right)^{+}$.

Ahora, $\alpha^{+} \in F_{x^{+}} \cap F_{y^{+}}$si y sólo si $\bar{\alpha} \in F_{x}$ y $\bar{\alpha} \in F_{y}$ si y sólo si $\alpha^{+}=\bar{\alpha}^{+} \in\left(F_{x} \cap F_{y}\right)^{+}$ y resulta:

10. $F_{x^{+}} \cap F_{y^{+}}=\left(F_{x} \cap F_{y}\right)^{+}$.

También tenemos que $x^{+} \in B_{\alpha^{+}} \Leftrightarrow \alpha^{+} \in F_{x^{+}} \Leftrightarrow \bar{\alpha} \in F_{x} \Leftrightarrow x \in B_{\bar{a}}$ :

11. $x^{+} \in B_{\alpha^{+}}$si y sólo si $x \in B_{\bar{\alpha}}$.

Si ahora $x^{+} \in\left(B_{\alpha}\right)^{+}$, tenemos que existe $y \in B_{\alpha}$ con $x^{+}=y^{+}$, pero entonces $\alpha \in F_{y}=F_{x}$ y por lo tanto, $x \in B_{\alpha}$. El recíproco es trivial y tenemos:

12. $x^{+} \in\left(B_{\alpha}\right)^{+}$si y sólo si $x \in B_{\alpha}$.

En vista de los dos incisos anteriores, observamos que la única diferencia posible entre $B_{\alpha^{+}} \mathrm{y}\left(B_{\bar{\alpha}}\right)^{+}$es que $B_{\alpha^{+}}$puede contener un vértice nuevo. Como $F_{G_{i}}=\left(G_{i}\right)^{+}$para toda $i$, tenemos que de hecho $B_{\alpha^{+}}$contiene a $G_{i+1}$ (siempre que $\alpha \in G_{i+1}$ ) siempre que tal vértice exista en $k(G)$. Renombrando $\alpha$ por $x$ y $G_{i+1}$ por $G_{i}$ resulta: 
13. Para toda $x \in G_{i}, B_{x^{+}}= \begin{cases}\left(B_{\bar{x}}\right)^{+} \cup\left\{G_{i}\right\} & \text { si } F G_{i}=B G_{i}=\varnothing \\ \left(B_{\bar{x}}\right)^{+} & \text {en caso contrario. }\end{cases}$

Ahora estamos listos para probar fácilmente el siguiente:

Teorema 3.3 Sea $G$ un reloj con segmentación $\mathcal{S}=\left\{G_{0}, G_{1}, \ldots, G_{s-1}\right\}$, entonces $k(G)$ es un reloj con segmentación $\widetilde{\mathcal{S}}=\left\{\tilde{G}_{0}, \tilde{G}_{1}, \ldots, \tilde{G}_{s-1}\right\}$ donde

$$
\tilde{G}_{i}= \begin{cases}\left\{x^{+}: x \in G_{i}\right\} \cup\left\{G_{i+1}\right\} & \text { si } F G_{i+1}=B G_{i+1}=\varnothing . \\ \left\{x^{+}: x \in G_{i}\right\} & \text { en caso contrario. }\end{cases}
$$

\section{Prueba.}

Usaremos libremente las afirmaciones numeradas precedentes. Ya hemos probado que $k(G)$ admite la segmentación $\widetilde{\mathcal{S}}$, de modo que sólo resta probar que también satisface las propiedades (I), (II) y (III) de los relojes.

Sabemos que $F_{x^{+}}=\left(F_{x}\right)^{+}$y $F_{G_{i}}=\left(G_{i}\right)^{+}$de donde se sigue que $k(G)$ satisface la propiedad (I), puesto que $F_{x}$ y $G_{i}$ son no vacíos por hipótesis.

Ahora supongamos que $u, v \in \tilde{G}_{i}$ y que $F_{u} \cap F_{v}=\varnothing$. Entonces, ninguno de los dos vértices puede ser igual a $G_{i+1}$ : $\mathrm{Si}$, por ejemplo, $u=G_{i+1}$ y $v=x^{+} \operatorname{con} x \in G_{i}$, tendríamos:

$$
F_{u} \cap F_{v}=F_{G_{i+1}} \cap F_{x^{+}}=\left(G_{i+1}\right)^{+} \cap\left(F_{x}\right)^{+} \supseteq\left(G_{i+1} \cap F_{x}\right)^{+}=\left(F_{x}\right)^{+} \neq \varnothing .
$$

Luego, sin pérdida de generalidad, $u=x^{+}$y $v=y^{+}$para algunas $x, y \in G_{i}$. Entonces, $\varnothing=F_{x^{+}} \cap F_{y^{+}}=\left(F_{x} \cap F_{y}\right)^{+}$implica que $F_{x} \cap F_{y}=\varnothing$. Como $G$ es un reloj, existe una $z \in G_{i}$ tal que $F_{z} \supseteq F_{x} \cup F_{y}$ y entonces:

$$
F_{z^{+}}=\left(F_{z}\right)^{+} \supseteq\left(F_{x} \cup F_{y}\right)^{+}=\left(F_{x}\right)^{+} \cup\left(F_{y}\right)^{+}=F_{x^{+}} \cup F_{y^{+}}
$$

y así hemos probado la propiedad (II).

Ahora, supongamos que $u, v \in \tilde{G}_{i}$ y que $F_{u} \cap F_{v} \neq \varnothing$.

Supongamos primero que uno de los vértices $u, v$ es un vértice nuevo, por ejemplo $u=G_{i+1}$ y $v=x^{+}$con $x \in G_{i}$. Entonces, como antes, tendríamos que $F_{u} \cap F_{v}=$ $F_{x^{+}}=F_{v}$. Como $B_{u}=B_{G_{i+1}}=\varnothing$, también es cierto que $B_{v} \supseteq B_{v}=B_{u} \cup B_{v}$. Luego, $v$ es el vértice de $k(G)$ que la propiedad (III) dice que debe existir. 
Finalmente veamos que pasa si ambos vértices $u \mathrm{y} v$ son vértices viejos, en este caso, $u=x^{+}$y $v=y^{+}$para algunas $x, y \in G_{i}$. Entonces:

$\varnothing \neq F_{x^{+}} \cap F_{y^{+}}=\left(F_{x} \cap F_{y}\right)^{+}$implica que $F_{\bar{x}} \cap F_{\bar{y}}=F_{x} \cap F_{y} \neq \varnothing$, luego, existe una $z \in G_{i}$ tal que $F_{z}=F_{\bar{x}} \cap F_{\bar{y}}$ y $B_{z} \supseteq B_{\bar{x}} \cup B_{\bar{y}}$. Entonces $F_{z^{+}}=\left(F_{z}\right)^{+}=\left(F_{\bar{x}} \cap F_{\bar{y}}\right)^{+}=$ $\left(F_{\bar{x}}\right)^{+} \cap\left(F_{\bar{y}}\right)^{+}=F_{\bar{x}^{+}} \cap F_{\bar{y}^{+}}=F_{x^{+}} \cap F_{y^{+}} \mathrm{y}\left(B_{\bar{z}}\right)^{+} \supseteq\left(B_{z}\right)^{+} \supseteq\left(B_{\bar{x}} \cup B_{\bar{y}}\right)^{+}=\left(B_{\bar{x}}\right)^{+} \cup$ $\left(B_{\bar{y}}\right)^{+}$. Ahora, si $F G_{i}=B G_{i}=\varnothing$ tenemos $B_{z^{+}}=\left(B_{\bar{z}}\right)^{+} \cup\left\{G_{i}\right\} \supseteq\left(B_{\bar{x}}\right)^{+} \cup\left(B_{\bar{y}}\right)^{+} \cup$ $\left\{G_{i}\right\}=B_{x^{+}} \cup B_{y^{+}}$y en caso contrario tenemos: $B_{z^{+}}=\left(B_{\bar{z}}\right)^{+} \supseteq\left(B_{\bar{x}}\right)^{+} \cup\left(B_{\bar{y}}\right)^{+}=$ $B_{x^{+}} \cup B_{y^{+}}$. Esto prueba que $k(G)$ satisface también (III).

Las funciones de $x \mapsto \bar{x}: V(G) \rightarrow V(G)$ y $x \mapsto x^{+}: V(G) \rightarrow V(k(G))$, nos permiten definir dos gráficas $\bar{G}$ y $G^{+}$como las subgráficas inducidas por la imagen de estas funciones en sus codominios naturales, es decir: $\bar{G}=G[\overline{V(G)}] \mathrm{y}$ $G^{+}=k(G)\left[V(G)^{+}\right]$y las restricciones de los codominios de estas funciones a sus respectivas imágenes nos dan dos nuevas funciones (que denotaremos con el mismo nombre): $x \mapsto \bar{x}: V(G) \rightarrow V(\bar{G})$ y $x \mapsto x^{+}: V(G) \rightarrow V\left(G^{+}\right)$. Es natural preguntarse si estas funciones son morfismos de gráficas. De hecho, son bastante más que eso:

Teorema 3.4 Si $G$ es un reloj con segmentación $\mathcal{S}=\left\{G_{0}, G_{1}, \ldots, G_{s-1}\right\}$, entonces $\bar{G}$ y $G^{+}$son relojes con segmentaciones $\overline{\mathcal{S}}=\left\{\bar{G}_{0}, \bar{G}_{1}, \ldots, \bar{G}_{s-1}\right\}$ y $\mathcal{S}^{+}=$ $\left\{G_{0}^{+}, G_{1}^{+}, \ldots, G_{s-1}^{+}\right\}$respectivamente. Además:

1. Las funciones $x \mapsto \bar{x}: G \rightarrow \bar{G}$ y $x \mapsto x^{+}: G \rightarrow G^{+}$son morfismos sobreyectivos plenos de gráficas.

2. Ambas funciones son morfismos de relojes (respetan las segmentaciones).

3. $x \mapsto \bar{x}: G \rightarrow \bar{G}$ es una retracción fuerte.

4. $\bar{G} \cong G^{+}$.

\section{Prueba.}

Mostremos primero que $\bar{G}$ es un reloj. En primer lugar, probaremos que si $x \in V(G)$ y $x \neq \bar{x}$ entonces $G-\{x\}$ es un reloj. Sea $G^{\prime}=G-\{x\}$ y sean $F^{\prime}$ y $B^{\prime}$ las funciones que describen las adyacencias en $G^{\prime}$. Sin pérdida de generalidad, supongamos que 
$x \in G_{i}$. Como $\bar{x} \in G_{i}$, tenemos que $G_{i}-\{x\} \supseteq\{\bar{x}\} \neq \varnothing$ y entonces $G-\{x\}$ es una gráfica segmentada con segmentos $G_{0}, \ldots, G_{i-1}, G_{i}-\{x\}, G_{i+1}, \ldots, G_{s-1}$. Si $v \in V\left(G^{\prime}\right)$ y $F_{v}^{\prime}=\varnothing$ entonces $F_{v}=\{x\}$, pero entonces, $\bar{x} \in F_{v}-\{x\}=F_{v}^{\prime}$ y por lo tanto $F_{v}^{\prime} \neq \varnothing$, contradicción. Observamos que $F_{u} \cap F_{v}=\varnothing$ si y sólo si $F_{u}^{\prime} \cap F_{v}^{\prime}=\varnothing$, pues si $x \in F_{u} \cap F_{v}$ entonces $\bar{x} \in F_{u} \cap F_{v}-\{x\}=F_{u}^{\prime} \cap F_{v}^{\prime}$. Si $F_{u}^{\prime} \cap F_{v}^{\prime}=\varnothing$ (ambas en el mismo segmento) entonces $F_{u} \cap F_{v}=\varnothing$ y existe una $w$ (en el mismo segmento que $u \mathrm{y}$ $v$ ) tal que $F_{w} \supseteq F_{u} \cup F_{v}$. En cualquier caso, $F_{\bar{w}}=F_{w} \supseteq F_{u} \cup F_{v}$ y $F_{\bar{w}}^{\prime} \supseteq F_{u}^{\prime} \cup F_{v}^{\prime}$ (note que si $w=x, w \notin V\left(G^{\prime}\right)$, pero siempre tenemos $\left.\bar{w} \in V\left(G^{\prime}\right)\right)$. Ahora supongamos que $F_{u}^{\prime} \cap F_{v}^{\prime} \neq \varnothing$. Entonces $F_{u} \cap F_{v} \neq \varnothing$ y existe una $w$ (en el mismo segmento que $u$ y $v$ ), tal que $F_{w}=F_{u} \cap F_{v}$ y $B_{w} \supseteq B_{u} \cup B_{v}$. De nuevo el problema es que $w$ puede no estar en $G^{\prime}$, sin embargo, $\bar{w} \in G^{\prime}$ tiene las propiedades: $F_{\bar{w}}=F_{w}=F_{u} \cap F_{v} \mathrm{y}$ $B_{\bar{w}} \supseteq B_{w} \supseteq B_{u} \cup B_{v}$. Luego $F_{\bar{w}}^{\prime}=F_{u}^{\prime} \cap F_{v}^{\prime}$ y $B_{\bar{w}}^{\prime} \supseteq B_{u}^{\prime} \cup B_{v}^{\prime}$. Hemos probado pues que $G^{\prime}$ es un reloj. Si ahora tomamos $y \in V(G)$ con $y \neq \bar{y}$ (en $G$ ) y $y \neq x$, tenemos que $F_{y}=F_{\bar{y}}$ y $B_{\bar{y}} \supseteq B_{y}$. Esto implica que $F_{y}^{\prime}=F_{\bar{y}}^{\prime}$ y $B_{\bar{y}}^{\prime} \supseteq B_{y}^{\prime}$ y podemos escoger la función $a \mapsto \bar{a}$ en $G^{\prime}$ de modo que $y \neq \bar{y}$ (en $G^{\prime}$ ). Finalmente, nos damos cuenta que podemos obtener $\bar{G}$ a partir de $G$ quitando uno a uno todos los vértices $x$ de $G$ que satisfacen $x \neq \bar{x}$, de modo que $\bar{G}$ es un reloj. Note que no necesariamente $\bar{G}=\bar{G}$. El hecho de que $G^{+}$es un reloj, quedará probado con la afirmación de que es isomorfo a $\bar{G}$.

Para cualesquiera dos vértices $x$ y $y$ adyacentes, es posible que ambos vértices estén en el mismo segmento o en segmentos consecutivos. Si $x, y \in G_{i}$ entonces $\bar{x}, \bar{y} \in G_{i} \mathrm{y}$ como todo segmento es completo, tenemos que $\bar{x}$ es adyacente a $\bar{y}$. Si por el contrario están en segmentos consecutivos, digamos, $x \in G_{i}$ y $y \in G_{i+1}$, entonces $y \in F_{x}=F_{\bar{x}}$ luego, $\bar{x} \in B_{y} \subseteq B_{\bar{y}}$ y entonces $\bar{x}$ y $\bar{y}$ son adyacentes. Con esto probamos que $x \mapsto \bar{x}$ es un morfismo de gráficas. La sobreyectividad y la plenitud se concluyen de inmediato por la definición de $\bar{G}$.

Si $x$ y $y$ son adyacentes, y están en el mismo segmento y entonces $x^{+}$y $y^{+}$también lo están y así, $x^{+}$y $y^{+}$también son adyacentes. Por el contrario, si están en segmentos consecutivos, digamos $x \in G_{i}$ y $y \in G_{i+1}$, entonces $x^{+} \cap y^{+}=B F_{y} \cap F_{x} \supseteq\{y\} \neq \varnothing$ y por lo tanto $x \mapsto x^{+}$es un morfismo de gráficas. La sobreyectividad se sigue de la definición de $G^{+}$. Para ver que es un morfismo pleno, supongamos que $x^{+}$ es adyacente a $y^{+}$, si ambos están en el mismo segmento, entonces $x$ y $y$ también lo están y por lo tanto son adyacentes. Supongamos que $x^{+} \in G_{i}^{+}$y $y^{+} \in \tilde{G}_{i+1}^{+}$, entonces $B_{x^{+}} \supseteq\left(B_{\bar{x}}\right)^{+} \ni y^{+}$, y por el enunciado con número 12,y $\in B_{\bar{x}}$. Luego, $\bar{x}$ 
y $y$ son adyacentes. Como $x^{+}=\bar{x}^{+}$(i.e $\bar{x}$ está en la preimagen de $x^{+}$) tenemos que $x \mapsto x^{+}$es un morfismo pleno.

Ambos morfismos respetan los segmentos por la forma en que están definidos ellos mismos y la forma en que están definidas $\bar{G}$ y $G^{+}$.

En general, tenemos que $N_{G}[x] \subseteq N_{G}[\bar{x}]$ para toda $x$. Luego, $x$ está dominado por $\bar{x}$, de modo si $x \neq \bar{x}, G-\{x\}$ es un retracto fuerte de $G$. Si $u$ y $v$ son ambos distintos de $x$ y $N_{G}[u] \subseteq N_{G}[v]$, tenemos que $N_{G-\{x\}}[u] \subseteq N_{G-\{x\}}[v]$. De modo que podemos seguir quitando vértices dominados de $G$ hasta obtener $\bar{G}$. Así hemos probado que $x \mapsto \bar{x}$ es una retracción fuerte.

Como $\bar{x}=\bar{y}$ si y sólo si $x^{+}=y^{+}$, la función $\bar{x} \mapsto x^{+}: V(\bar{G}) \rightarrow V\left(G^{+}\right)$está bien definida y es biyectiva. Es un isomorfismo porque $F_{\bar{x}^{+}}=\left(F_{\bar{x}}\right)^{+}$y por el enunciado con número 7 .

Ahora podemos probar el siguiente:

Teorema 3.5 Si $G$ es un reloj, podemos obtener (una copia isomorfa a) $k(G)$ con dos simples operaciones:

tic: Calcular $\bar{G}$. Es decir, para cada vértice $x$ en $G_{i}$ escoger un vértice $\bar{x}$, con las propiedades: $F_{x}=F_{\bar{x}}, F_{x}=F_{y} \Rightarrow B_{\bar{x}} \supseteq B_{y}$ y $F_{x}=F_{y} \Rightarrow \bar{x}=\bar{y}$ y luego tomamos $\bar{G}$ como la subgráfica inducida en $G$ por estos vértices $\{\bar{x}: x \in G\}$.

tac: Agregar un nuevo vértice a cada segmento $\bar{G}_{i}$ de $\bar{G}$ siempre que $G_{i}$ sea un segmento bueno y agregar las aristas necesarias para cada uno de estos vértices nuevos sea adyacente a todos los vértices viejos de su propio segmento y del siguiente.

Prueba. Inmediato de los teoremas 3.3 y 3.4 .

En el caso $G=\bar{G}$, también es útil, para calcular gráficas iteradas de clanes el siguiente resultado:

Teorema 3.6 Si $G=\bar{G}$ entonces $k(G)=\overline{k(G)}$. 


\section{Prueba.}

La condición $G=\bar{G}$ es equivalente a que para cualesquiera $x, y \in V(G), F_{x}=F_{y} \Rightarrow$ $x=y$. Probaremos que esta última condición se satisface en $k(G)$.

Como $G=\bar{G} \cong G^{+}$, tenemos que la función $x \mapsto x^{+}: G \rightarrow G^{+}$es inyectiva y entonces resulta que:

$$
F_{x^{+}}=F_{y^{+}} \Rightarrow\left(F_{x}\right)^{+}=\left(F_{y}\right)^{+} \Rightarrow F_{x}=F_{y} \Rightarrow x=y \Rightarrow x^{+}=y^{+} .
$$

También se tiene que:

$$
F_{G_{i}}=F_{G_{j}} \Rightarrow\left(G_{i}\right)^{+}=\left(G_{j}\right)^{+} \Rightarrow G_{i}=G_{j} .
$$

Finalmente, usando de nuevo la inyectividad de +:

$$
F_{G_{i}}=F_{x^{+}} \Rightarrow\left(G_{i}\right)^{+}=\left(F_{x}\right)^{+} \Rightarrow G_{i}=F_{x} \Rightarrow B G_{i} \supseteq\{x\} \neq \varnothing .
$$

Pero si $B G_{i} \neq \varnothing$ entonces $G_{i}$ no es un clan de $G$ y tampoco es un vértice de $k(G)$.

\section{El $k$-carácter de los relojes}

Teorema 3.7 Si $G$ es un reloj con segmentación $\mathcal{S}=\left\{G_{0}, G_{1}, \ldots, G_{s-1}\right\}$ y $F G_{i} \neq$ $\varnothing$ para alguna $i$, entonces $F G_{j} \neq \varnothing$ para toda $j$. Con la segmentación usual $\widetilde{\mathcal{S}}=$ $\left\{\tilde{G}_{0}, \tilde{G}_{1}, \ldots, \tilde{G}_{s-1}\right\}, k(G)$ también satisface $F \tilde{G}_{j} \neq \varnothing$ para toda $j$.

\section{Prueba.}

Si $F G_{i} \neq \varnothing$, entonces, por la propiedad (III) de los relojes, existe $z \in G_{i}$ tal que $F_{z}=F G_{i}$ y $B_{z} \supseteq \bigcup_{x \in G_{i}} B_{x}$ pero, como $F_{y} \neq \varnothing$ para toda $y \in G_{i-1}$ (de hecho para toda $x \in V(G)$ ), tenemos: $\bigcup_{x \in G_{i}} B_{x}=G_{i-1}$. Entonces $B_{z}=G_{i-1}$ y $z \in F G_{i-1}$, por lo que $F G_{i-1} \neq \varnothing$. Repitiendo este proceso otras $s-2$ veces, tenemos la primera afirmación que había que probar.

Ahora, ningún $G_{j}$ puede ser clan de $G$, de modo que usando el el enunciado con número 10 de la sección anterior, tenemos: $F \tilde{G}_{i}=\bigcap_{x \in G_{i}} F_{x^{+}}=\bigcap_{x \in G_{i}}\left(F_{x}\right)^{+}=$ $\left(\bigcap_{x \in G_{i}} F_{x}\right)^{+}=\left(F G_{i}\right)^{+}$. Pero sabemos que $F G_{i} \neq \varnothing$ para toda $i$. 
Teorema 3.8 Si $G$ es un reloj con segmentación $\mathcal{S}=\left\{G_{0}, G_{1}, \ldots, G_{s-1}\right\}, F G_{i}=\varnothing$ para toda $i$ y $k(G)$ tiene la segmentación usual $\widetilde{\mathcal{S}}=\left\{\tilde{G}_{0}, \tilde{G}_{1}, \ldots, \tilde{G}_{s-1}\right\}$, entonces:

1. $F \tilde{G}_{i}=\varnothing$ para toda $i$.

2. $B G_{i+1}=\varnothing$ si y sólo si $B \tilde{G}_{i}=\varnothing$.

3. $G$ y $k(G)$ tienen el mismo número de segmentos buenos.

\section{Prueba.}

Dependiendo de si $B G_{i+1}=\varnothing$ o no, tenemos $F \tilde{G}_{i}=\left(\bigcap_{x \in G_{i}} F_{x^{+}}\right) \cap F_{G_{i+1}}$ o $F \tilde{G}_{i}=$ $\bigcap_{x \in G_{i}} F_{x^{+}}$, pero $\left(\bigcap_{x \in G_{i}} F_{x^{+}}\right) \cap F_{G_{i+1}}=\left(\bigcap_{x \in G_{i}} F_{x^{+}}\right) \cap\left(G_{i+1}\right)^{+}=\bigcap_{x \in G_{i}} F_{x^{+}}$y entonces, en cualquier caso resulta $F \tilde{G}_{i}=\bigcap_{x \in G_{i}} F_{x^{+}}=\bigcap_{x \in G_{i}}\left(F_{x}\right)^{+}=\left(\bigcap_{x \in G_{i}} F_{x}\right)^{+}=$ $\left(F G_{i}\right)^{+}$de modo que $F \tilde{G}_{i}=\varnothing$ para toda $i$.

Si $B G_{i+1}=\varnothing$, tenemos que $B \tilde{G}_{i}=\left(\bigcap_{x \in G_{i}} B_{x^{+}}\right) \cap B_{G_{i+1}} \subseteq B_{G_{i+1}}=\varnothing$.

Si por el contrario, $B G_{i+1} \neq \varnothing$, resulta que o bien $B G_{i} \neq \varnothing$ y $B \tilde{G}_{i}=\bigcap_{x \in G_{i}} B_{x^{+}}=$ $\bigcap_{x \in G_{i}}\left(B_{\bar{x}}\right)^{+} \supseteq\left(\bigcap_{x \in G_{i}} B_{\bar{x}}\right)^{+} \supseteq\left(\bigcap_{x \in G_{i}} B_{x}\right)^{+}=\left(B G_{i}\right)^{+} \neq \varnothing$ o bien $B G_{i}=\varnothing, \mathrm{y}$ entonces $B \tilde{G}_{i}=\bigcap_{x \in G_{i}} B_{x^{+}}=\bigcap_{x \in G_{i}}\left(\left(B_{\bar{x}}\right)^{+} \cup\left\{G_{i}\right\}\right) \supseteq\left\{G_{i}\right\} \neq \varnothing$.

La tercera afirmación es consecuencia inmediata de las primeras dos.

Definimos $\stackrel{\infty}{\bar{G}}$ como la gráfica que se obtiene de $G$ al aplicar la operación $H \mapsto \bar{H}$ un número infinito de veces. Esta gráfica nos permite decidir sobre la divergencia de relojes:

Teorema 3.9 Un reloj $G$ con segmentación $\mathcal{S}=\left\{G_{0}, G_{1}, \ldots, G_{s-1}\right\}$ esk-divergente si y sólo si $\stackrel{\infty}{G}$ tiene algún segmento bueno.

\section{Prueba.}

Por comodidad, renombramos $\stackrel{\infty}{\bar{G}}$ por $G^{\prime}$. Como $\bar{G}$ es un retracto fuerte de $G, G^{\prime}$ también es un retracto fuerte de $G$, por el teorema $1.11, G^{\prime}$ y $G$ tienen el mismo $k$-carácter. Sean $\left\{G_{i}^{\prime}\right\}$ los segmentos de $G^{\prime}$ y sea $F^{\prime}$ la función que describe las adyacencias en $G^{\prime}$. Sabemos que $\overline{G^{\prime}}=G^{\prime}$. Si $F^{\prime} G_{i}^{\prime} \neq \varnothing$, para alguna $i$, tenemos que 
$G^{\prime}$ no puede tener segmentos buenos y $k\left(G^{\prime}\right) \cong \overline{G^{\prime}}=G^{\prime}$ (note que no necesariamente $k(G) \cong G)$. Por lo que $G^{\prime}$ tiene que ser $k$-convergente.

Si $F^{\prime} G_{i}^{\prime}=\varnothing$ para toda $i$ y $m$ es el número de segmentos buenos de $G^{\prime}$, tenemos que, $\left|k\left(G^{\prime}\right)\right|=\left|\overline{G^{\prime}}\right|+m$. Por el teorema anterior, sabemos que la condición $F^{\prime} G_{i}^{\prime}=\varnothing$ y el número de segmentos buenos se conservan al calcular la gráfica de clanes, de modo que $\left|k^{n}\left(G^{\prime}\right)\right|=\left|G^{\prime}\right|+n m$ y así $G^{\prime}$ es $k$-divergente.

Como la operación $G \mapsto \bar{G}$ es polinomial y hay que aplicar esta operación un máximo de $|G|$ veces, tenemos el siguiente:

Teorema 3.10 Si $G$ es un reloj, podemos calcular en tiempo polinomial el $k$-carácter de $G$.

Un resultado análogo para cografos será publicado en [37]. Pero el problema de calcular el $k$-carácter para gráficas en general parece mucho más difícil, de hecho, ni siquiera se sabe si existe algún algoritmo (no necesariamente polinomial) que calcule el $k$-carácter.

Problema 5 (Problema de la computabilidad del $k$-carácter [43]) ¿Es computable el k-carácter?

Observamos que existe un algoritmo que calcula el $k$-carácter si y sólo si existe un algoritmo que decide la $k$-divergencia. Por otro lado, un algoritmo que decida la $k$-nulidad no sería suficiente para calcular el $k$-carácter y de nuevo, nadie sabe si tal algoritmo existe:

Problema 6 (Problema de la computabilidad de la $k$-nulidad) [43] ¿Es computable la $k$-nulidad?

El problema 5 puede ser un reto incluso en instancias particulares. Todas las técnicas conocidas no son suficientes para determinar el $k$-carácter de la gráfica de la figura 3.1. La importancia de este ejemplo en particular quedará claro cuando enunciemos el problema 16 (pág. 82).

Problema 7 Determinar el $k$-carácter de la gráfica de la figura 3.1. 


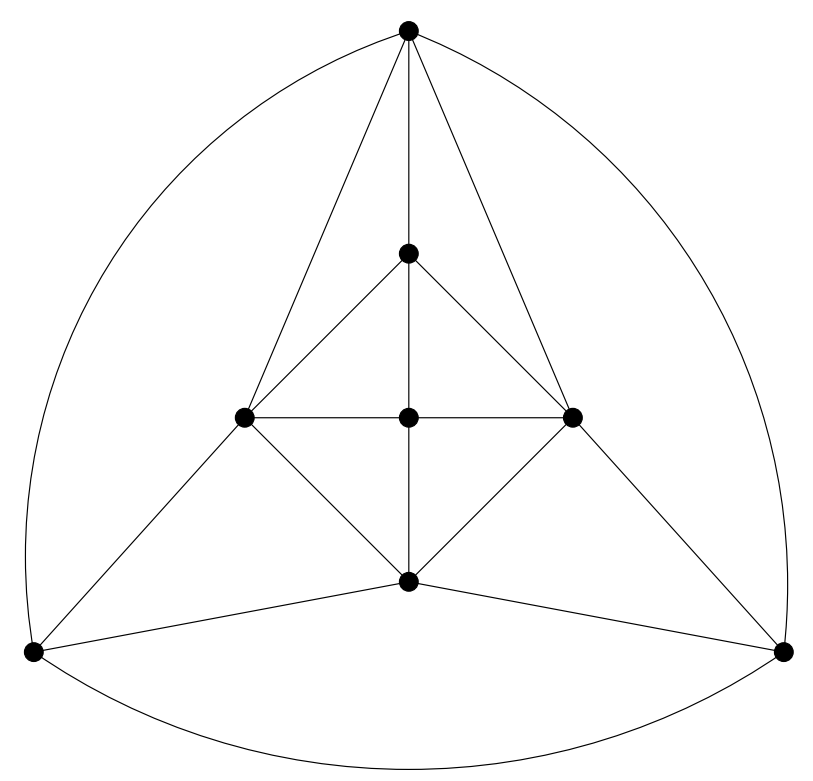

Figura 3.1: Una gráfica de $k$-carácter desconocido

\section{Sumas segmentadas}

Conviene hacer una pausa aquí, para revisar los relojes originales (tal y como fueron definidos en $[41,43])$ y su relación con los relojes aquí definidos.

Sean $M$ y $N$ dos gráficas cíclicamente segmentadas disjuntas con segmentaciones $\mathcal{M}=\left\{M_{0}, M_{1}, \ldots, M_{s-1}\right\}$ y $\mathcal{N}=\left\{N_{0}, N_{1}, \ldots, N_{s-1}\right\}$ respectivamente. Definimos la suma segmentada $M \boxplus N$ de $M$ y $N$ como la gráfica cíclicamente segmentada $G$ con segmentación $\mathcal{G}=\left\{G_{0}, G_{1}, \ldots, G_{s-1}\right\}$ dada por $G_{i}=M_{i} \cup N_{i}$, que tiene $V(G)=$ $V(M) \cup V(N)$ y $E(G)=\left\{\{u, v\}: u \in M_{i}, v \in N_{j}\right.$ con $i=j$ ó $\left.i=j+1\right\} \cup E(M) \cup$ $E(N)$. Es una sencilla verificación mostrar que en efecto $G=M \boxplus N$ es una gráfica cíclicamente segmentada. Observamos que la suma segmentada no es conmutativa, pero sí es asociativa.

A parte de las propiedades (I), (II) y (III) de los relojes, vamos a necesitar considerar una variante de la propiedad (III):

(IIIa) Para toda $G_{i} \in \mathcal{S}$ y para toda $x, y \in G_{i}$ existe una $z \in G_{i}$ tal que:

$$
F_{z}=F_{x} \cap F_{y} \text { y } B_{z} \supseteq B_{x} \cup B_{y} .
$$

La condición (IIIa) es equivalente a pedir la condición (III) y también pedir que en 
caso de que $F_{x} \cap F_{y}=\varnothing$ para algunas $x, y \in G_{i}$, entonces también exista una $z$ con $F_{z}=\varnothing$ y $B_{z} \supseteq B_{w}$ para toda $w \in G_{i}$.

Teorema 3.11 Sean $M$ y $N$ gráficas cíclicamente segmentadas con segmentaciones $\mathcal{M}=\left\{M_{0}, M_{1}, \ldots, M_{s-1}\right\}$ y $\mathcal{N}=\left\{N_{0}, N_{1}, \ldots, N_{s-1}\right\}$ respectivamente. Supongamos que $M$ satisface (I) y (III) y que $N$ satisface (IIIa). Entonces $M \boxplus N$ es un reloj.

Prueba. Sea $G=M \boxplus N$, probaremos que $G$ satisface (I), (II) y (III).

(I): Si $x \in G_{i}$ tenemos que $F_{x} \neq \varnothing$ ya sea porque $x \in M_{i}$ y $M$ satisface (I) o por que $x \in N_{i}$ y $F_{x} \supseteq M_{i+1} \neq \varnothing$.

(II): Sean $x, y \in G_{i}$ y supongamos que $F_{x} \cap F_{y}=\varnothing$. Como $M$ satisface (I) y $F_{v} \subseteq M_{i+1}$ siempre que $v \in N_{i}$, se sigue que tanto $x$ como $y$ pertenecen a $M_{i}$. Entonces, cualquier $z$ en $N_{i}$ satisface $F_{z} \supseteq F_{x} \cup F_{y}$.

(III): Sean $x, y \in G_{i}$ y supongamos que $F_{x} \cap F_{y} \neq \varnothing$. Si (digamos) $x \in M_{i}$ y $y \in N_{i}$, como $F_{x} \subseteq F_{y}$ y $B_{x} \supseteq B_{y}$, tomando $z=x$ tenemos: $F_{z}=F_{x} \cap F_{y}$ y $B_{z} \supseteq B_{x} \cup B_{y}$ como queríamos. Si ahora suponemos que $x, y \in M_{i}$, la $z$ que existe en $M_{i}$ dado que $M$ satisface (III) nos sirve aquí. Finalmente, consideremos el caso $x, y \in N_{i}$. La $z$ que existe en $N_{i}$ dado que $N$ satisface (IIIa) es la $z$ que buscamos (Note que es posible que $F_{x} \cap F_{y} \cap N_{i+1}=\varnothing$, de modo que la propiedad (III) no sería suficiente aquí).

Una manera sencilla y natural de lograr que $M$ satisfaga (III) es prohibiendo que la condición $F_{x} \cap F_{y} \neq \varnothing$ tenga lugar. Si agregamos ahora la condición (I), $M$ se ve obligada a que todos sus segmentos tengan el mismo orden y que cada vértice en $M_{i}$ sea vecino de exactamente un vértice en $M_{i+1}$ para toda $i$. A una gráfica cíclicamente segmentada como ésta se le llama en $[41,43]$ corona (de un reloj).

Por otro lado, también una manera sencilla de lograr que $N$ satisfaga (IIIa) es dando un orden lineal $x_{1}<x_{2}<\cdots<x_{n_{i}}$ dentro de cada segmento $N_{i}=\left\{x_{1}, \ldots, x_{n_{i}}\right\}$ de $N$ y luego pidiendo que cada vez que $x<y$ tengamos que $F_{x} \subseteq F_{y}$ y $B_{y} \subseteq B_{x}$. A una gráfica cíclicamente segmentada como ésta se le llama en [41,43] núcleo (de un reloj).

En $[41,43]$ se definen los relojes (que aquí llamaremos relojes antiguos) como la suma segmentada de una corona y un núcleo. Gracias al teorema anterior sabemos que 
todo reloj antiguo es un reloj:

Corolario 3.12 Si $M$ es una corona y $N$ es un núcleo, cada uno con s segmentos entonces $M \boxplus N$ es un reloj.

Es claro ya en este contexto que no todos los relojes son relojes antiguos (por ejemplo, un núcleo que satisfaga (I) es reloj, pero no es antiguo). Sin embargo subsiste el hecho de que los relojes antiguos son más fáciles de construir y son una subclase amplia e interesante de los relojes. Además, se pueden calcular sus gráficas de clanes muy fácilmente pues para un reloj antiguo la corona se mantiene invariante y sólo debemos concentrarnos en lo que sucede en su núcleo.

Todos los relojes que presentamos en la siguiente sección son antiguos y sus coronas tienen 2 vértices en cada segmento.

\section{Ejemplos}

Como dijimos, los relojes son útiles para construir ejemplos y contraejemplos. Un primer interesante ejemplo es el de una gráfica $k$-invariante que no es clan-Helly:

Ejemplo 3.13 El reloj $R_{0}$ con vértices $\{1, \ldots, 13\}$ y segmentación

$$
\mathcal{S}=\{\{1,2,3,4\},\{5,6,7\},\{8,9,10\},\{11,12,13\}\}
$$

que se muestra en la figura 3.2 es una gráfica $k$-invariante que no es clan-Helly

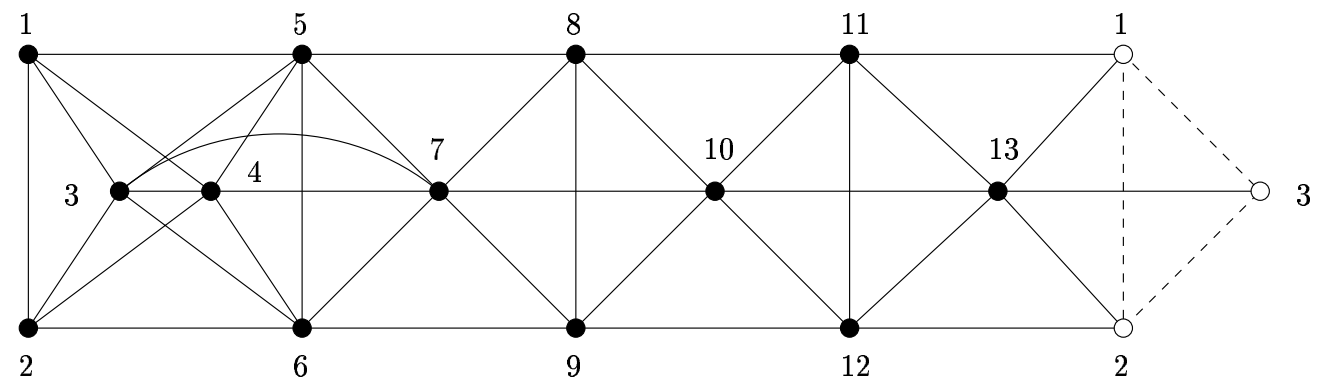

Figura 3.2: Una gráfica $k$-invariante que no es clan-Helly 
De hecho, el ejemplo previo fue presentado por Escalante en [15] (1973), muchos otros ejemplos pueden construirse hoy en día, gracias a los relojes. Sorprendentemente, la suma punteada de dos gráficas $k$-invariantes puede ser clan divergente:

Ejemplo 3.14 La gráfica que se obtiene del reloj del ejemplo anterior al tomar la suma puntada (en el vértice 4 ) con un cuadrado, $G=\left(R_{0}, 4\right) \cdot\left(\mathcal{C}_{4}, 1\right)$ es una gráfica clan divergente y los órdenes de sus gráficas iteradas de clanes están dados por $\left|k^{n}(G)\right|=|G|+\left\lceil\frac{n}{2}\right\rceil$.

Aunque la gráfica del ejemplo anterior no es un reloj, usando la maquinaria desarrollada para los relojes, es fácil probar que lo que se afirma en él es en efecto cierto: cada $k^{2 n}(G)$ es la suma punteada de un reloj y un cuadrado, de hecho, $k^{2 n}(G) \cong \operatorname{tac}^{2 n}\left(R_{0}\right) \cdot \mathcal{C}_{4}$. Nos preguntamos si algo semejante puede pasar para gráficas $k$-nulas.

Problema 8 (Frías y Neumann-Lara [17]) ¿Es cierto que la suma punteada de dos gráficas $k$-nulas es $k$-nula?

Otra sorpresa que que plantea el ejemplo 3.14 es que es posible que la función de crecimiento definida por $f_{G}(n)=\left|k^{n}(G)\right|$ puede ser menor que $|G|+n$ para una gráfica divergente $G$.

Problema 9 ¿Cuál es la mínima función de crecimiento posible para una gráfica clan divergente?

Dos ejemplos serán imprescindibles para el siguiente capítulo.

Ejemplo 3.15 Definimos el reloj $T_{s}^{n}$, para $s \geq 4, n \geq 0$ como aquel que tiene por vértices a $V=\left\{x_{j}^{i}: i \in \mathbb{Z}_{s}, j \in\{0, \ldots, n+2\}\right\}$, segmentación $\mathcal{S}=\left\{G_{i}: i \in \mathbb{Z}_{s}\right\}$, con $G_{i}=\left\{x_{j}^{i}: j \in\{0, \ldots, n+2\}\right\}$ y adyacencias dadas por:

$$
\begin{aligned}
& F_{x_{0}^{i}}=\left\{x_{0}^{i+1}\right\}, F_{x_{1}^{i}}=\left\{x_{1}^{i+1}\right\} \\
& F_{x_{j}^{i}}=\left\{x_{j^{\prime}}^{i+1}: j^{\prime}<j\right\} \text { siempre que } j \neq 0,1 .
\end{aligned}
$$

Entonces, tenemos que $\overline{T_{s}^{n}}=T_{s}^{n}, F G_{i}=B G_{i}=\varnothing$ para toda $i, T_{s}^{n}$ tiene s segmentos buenos, $k\left(T_{s}^{n}\right) \cong T_{s}^{n+1} y\left|k^{n}\left(T_{s}^{0}\right)\right|=3 s+n s$. En particular, $T_{s}^{n}$ es $k$-divergente. 

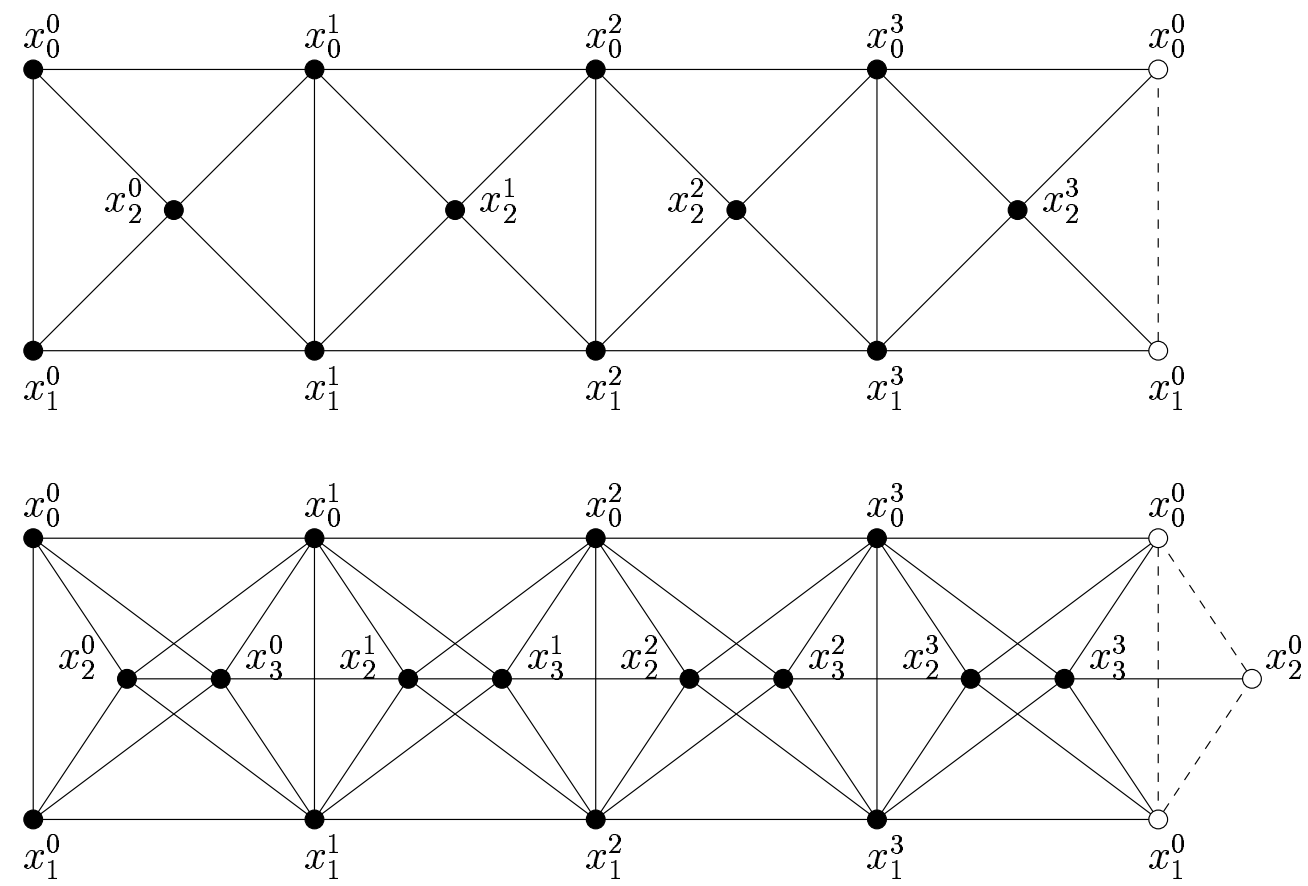

Figura 3.3: De arriba a abajo: $T_{4}^{0}$ y $T_{4}^{1}$

Ejemplo 3.16 Definimos el reloj $H_{m}^{n}$, para $m \geq 2, n \geq 0$ como aquél que tiene por vértices a

$$
V=\left\{x_{j}^{i}: i \in\{0, \ldots, 2 m-1\}, j \in\left\{0, \ldots, 2+\left\lfloor\frac{n+(i \bmod m)}{m}\right\rfloor\right\}\right\}
$$

segmentación $\mathcal{S}=\left\{G_{i}: i \in\{0, \ldots, 2 m-1\}\right\}$, con

$$
G_{i}=\left\{x_{j}^{i}: j \in\left\{0, \ldots, 2+\left\lfloor\frac{n+(i \bmod m)}{m}\right\rfloor\right\}\right\}
$$

y adyacencias dadas por:

$$
F_{x_{j}^{i}}= \begin{cases}\left\{x_{j}^{i+1}\right\} & \text { si } j \in\{0,1\}, \\ \left\{x_{j^{\prime}}^{i+1}: j^{\prime}<j\right\} & \text { si } j \notin\{0,1\}, i \in\{m-1,2 m-1\}, \\ \left\{x_{j^{\prime}}^{i+1}: j^{\prime} \leq j\right\} & \text { si } j \notin\{0,1\}, i \notin\{m-1,2 m-1\} .\end{cases}
$$

Recuerde que, como tenemos $2 m$ segmentos, los índices $i$ se toman módulo $2 m$. Entonces tenemos $\overline{H_{m}^{n}}=H_{m}^{n}, F G_{i}=\varnothing$ para toda $i$ y $B G_{i}=\varnothing$ si y sólo si $i+n \equiv m-1$ $\bmod m . H_{m}^{n}$ tiene 2 segmentos buenos, $k\left(H_{m}^{n}\right) \cong H_{m}^{n+1} y\left|k^{n}\left(H_{m}^{0}\right)\right|=6 m+2 n$. En particular, $H_{m}^{n}$ es $k$-divergente. 

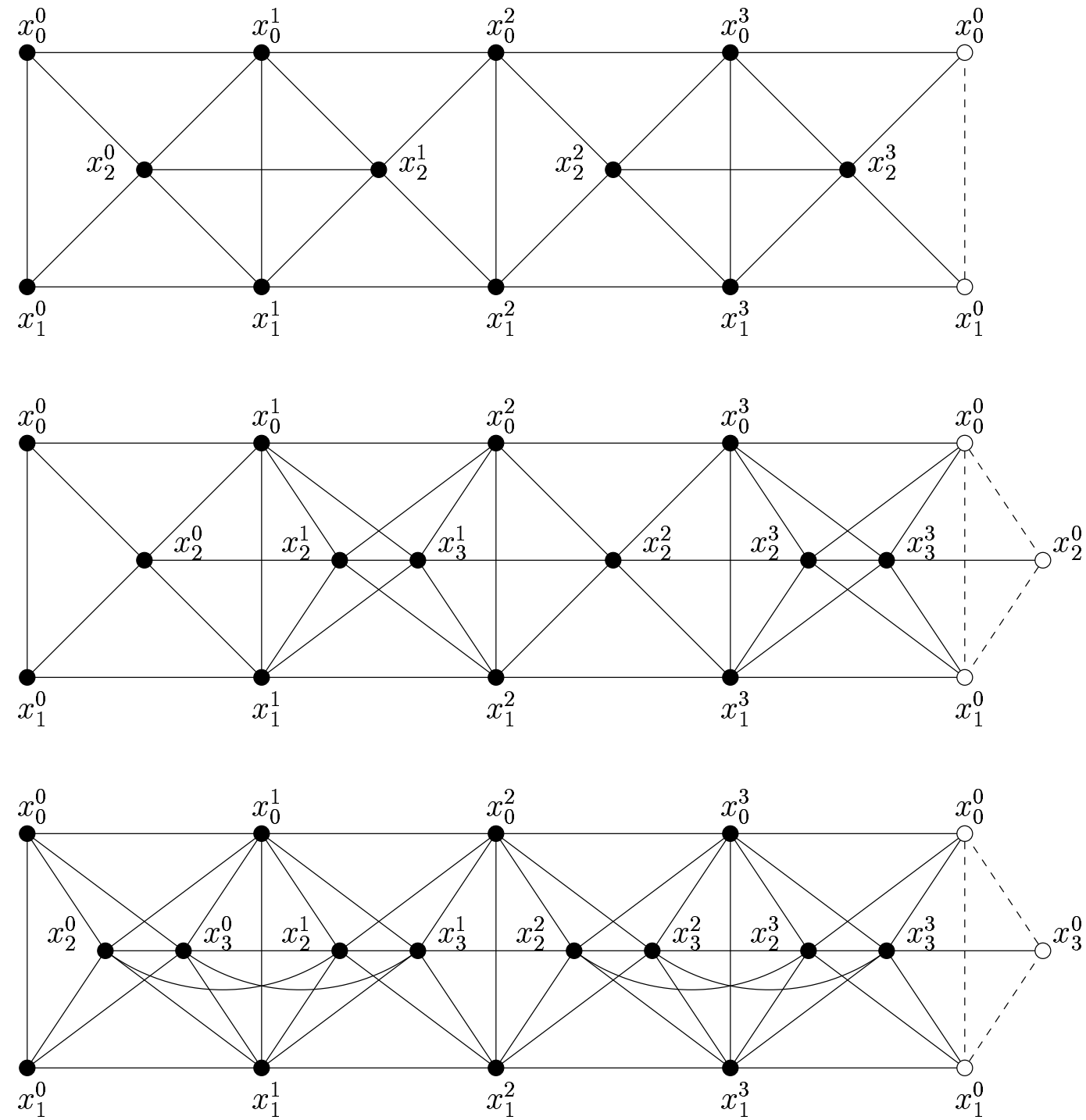

Figura 3.4: De arriba a abajo: $H_{2}^{0}, H_{2}^{1}$ y $H_{2}^{2}$ 


\section{Capítulo 4}

\section{Gráficas coafines}

En este capítulo estudiaremos las gráficas divergentes en sumandos que resultan ser una generalización de las gráficas expansivas de Neumann-Lara $[55,57]$ y de las gráficas absolutamente saturadas [61]. Con estas nuevas técnicas podremos resolver el problema del $k$-carácter del icosaedro $[40,57,58]$ que permaneció abierto por 20 años. También probaremos que toda superficie (cerrada) admite una triangulación $k$-divergente.

Las primeras tres secciones de este capítulo, presentan los conceptos y resultados de Neumann-Lara $[54,55,57]$ que usaremos. Incluimos pruebas para la comodidad del lector.

\section{Las categorías $\mathcal{G}, \mathcal{G}^{a}, \mathcal{G}^{(r)}$}

Como se dijo en la introducción, un morfismo entre dos gráficas $A$ y $B$ es una función $f: V(A) \rightarrow V(B)$ que manda vértices adyacentes en vértices adyacentes o iguales. La categoría de las gráficas, $\mathcal{G}$, se define como aquélla que tiene por objetos a las gráficas con los morfismos descritos.

Una gráfica automorfa $\mathbb{A}$ es un par ordenado $\left(A, \tau_{A}\right)$ donde $A$ es una gráfica y $\tau_{A}$ es un automorfismo de $A$. Un morfismo entre dos gráficas automorfas $\mathbb{A}=\left(A, \tau_{A}\right) \mathrm{y}$ $\mathbb{B}=\left(B, \tau_{B}\right)$ es un morfismo admisible entre $A$ y $B$, es decir, un morfismo de gráficas $f: A \rightarrow B$ (las imágenes de vértices adyacentes son adyacentes o iguales) tal que 
$f \circ \tau_{A}=\tau_{B} \circ f$. La categoría de las gráficas automorfas, $\mathcal{G}^{a}$, es la que tiene a los objetos y morfismos recién descritos. A veces, cuando el contexto sea suficientemente claro, llamaremos "gráfica" a una "gráfica automorfa" y "morfismo" a un "morfismo admisible".

Decimos que $\mathbb{A}=\left(A, \tau_{A}\right)$ es una subgráfica (automorfa) de $\mathbb{B}=\left(B, \tau_{B}\right)$ si $A$ es subgráfica de $B, \tau_{B}(A)=A$ y $\tau_{A}$ es la restricción de $\tau_{B}$ a $A$. Si $f: \mathbb{A} \rightarrow \mathbb{B}$ es un morfismo en $\mathcal{G}^{a}$ con $\mathbb{A}=\left(A, \tau_{A}\right)$ y $\mathbb{B}=\left(B, \tau_{B}\right)$ definimos $f(\mathbb{A})=\left(f(A), \tau_{f(A)}\right)$ donde $\tau_{f(A)}$ es la restricción de $\tau_{B}$ a $f(A)$. Claramente $f(\mathbb{A})$ es una subgráfica de $\mathbb{B}$ y $f$ se restringe a un morfismo de $\mathbb{A}$ en $f(\mathbb{A})$. También debe ser claro que si $\mathbb{C}=\left(C, \tau_{C}\right)$ es una subgráfica de $\mathbb{B}$, entonces $f^{-1}(\mathbb{C})=\left(f^{-1}(C), \tau_{f^{-1}(C)}\right)$ (donde $\tau_{f^{-1}(C)}$ es la restricción de $\tau_{A}$ a $\left.f^{-1}(C)\right)$ es una subgráfica de $\mathbb{A}$.

Si $r \geq 2$, decimos que una gráfica automorfa $\mathbb{A}$ es $r$-coafín si $d_{A}\left(x, \tau_{A}(x)\right) \geq r$ para todo $x \in A$. También decimos simplemente que $\mathbb{A}$ es coafín en caso de que el valor de $r$ sea claro o no importante. Definimos la categoría de gráficas $r$-coafines, $\mathcal{G}^{(r)}$, como la subcategoría plena de $\mathcal{G}^{a}$ que resulta de restringir los objetos a las gráficas $r$-coafines. Note que $\mathcal{G}^{(r+1)}$ es una subcategoría plena de $\mathcal{G}^{(r)}$.

Las pruebas de que las categorías descritas en efecto lo son, se omiten por ser verificaciones rutinarias.

Los morfismos completos van a ser fundamentales en el estudio de las gráficas iteradas de clanes:

Definición 4.1 En cualquiera de las categorías $\mathcal{G}, \mathcal{G}^{a}, \mathcal{G}^{(r)}$, decimos que un morfismo $\tau: A \rightarrow B$ es completo si para cada subgráfica completa $S$ de $B, \tau^{-1}(S)$ es una subgráfica completa de $A$.

Note que basta verificar que $\tau^{-1}(S)$ es completa cuando $|S| \leq 2$.

El producto fuerte de gráficas automorfas, $\mathbb{A} \otimes \mathbb{B}$ se define simplemente como $\mathbb{A} \otimes \mathbb{B}=$ $\left(A \otimes B, \tau_{A} \times \tau_{B}\right)$. Es claro que este producto fuerte también está bien definido para gráficas $r$-coafines (ver teorema 1.2) y que tanto en $\mathcal{G}^{a}$ como en $\mathcal{G}^{(r)}$ se trata del producto categórico. 


\section{El operador de clanes en $\mathcal{G}, \mathcal{G}^{a}, \mathcal{G}^{(r)}$}

El operador $k$ puede definirse de manera natural en todas las categorías descritas. Ya lo definimos previamente en $\mathcal{G}$. Sea $\mathbb{A}=\left(A, \tau_{A}\right) \in \mathcal{G}^{a}$ definimos $k(\mathbb{A})=\left(k(A), \tau_{k(A)}\right)$ donde $\tau_{k(A)}$ se define como $\tau_{k(A)}(Q)=\left\{\tau_{A}(x): x \in Q\right\}$ para toda $Q \in k(A)$. Hace falta mostrar que $\tau_{k(A)}$ es en efecto un automorfismo de $k(A)$ :

En primer lugar, veamos que $\tau_{k(A)}(Q) \in k(A)$. Note que todo morfismo de gráficas manda subgráficas completas en subgráficas completas, así que $\tau_{k(A)}(Q)=$ $\left\{\tau_{A}(x): x \in Q\right\}$ es una subgráfica completa de $A$. Sea $Q^{\prime}$ un clan de $A$ que contiene a $\tau_{k(A)}(Q)$ entonces $Q=\tau_{k(A)}^{-1} \circ \tau_{k(A)}(Q) \subseteq \tau_{k(A)}^{-1}\left(Q^{\prime}\right)$. Como $Q$ es clan tenemos $Q=\tau_{k(A)}^{-1}\left(Q^{\prime}\right)$ y por lo tanto $\tau_{k(A)}(Q)=Q^{\prime}$, de modo que $\tau_{k(A)}(Q)$ es maximal y por tanto es un clan de $A$.

Sólo resta probar que $\tau_{k(A)}$ preserva adyacencias. Sean $Q$ y $Q^{\prime}$ dos clanes de $A$ adyacentes en $k(A)$, es decir, $Q \cap Q^{\prime} \neq \varnothing$. Entonces

$$
\tau_{k(A)}(Q) \cap \tau_{k(A)}\left(Q^{\prime}\right) \supseteq\left\{\tau_{A}(x): x \in Q \cap Q^{\prime}\right\} \neq \varnothing
$$

Así, hemos probado que $\tau_{k(A)}$ es un automorfismo de $k(A)$ y por lo tanto que $k$ también es un operador en $\mathcal{G}^{a}$. En lo sucesivo omitiremos los subíndices de $\tau_{A} \mathrm{y} \tau_{k(A)}$ siempre que ello no cause confusión.

Como $\mathcal{G}^{(r)}$ es una subcategoría de $\mathcal{G}^{a}$, para ver que $k$ también es un operador en $\mathcal{G}^{(r)}$ basta ver que si $\mathbb{A}$ es $r$-coafín entonces $k(\mathbb{A})$ también lo es. Sea $\mathbb{A}=(A, \tau)$ una gráfica automorfa $r$-coafín y sea $Q \in k(A)$. En primer lugar notamos que si $d(x, y) \leq 1$ para algunas $x, y \in A$, por la desigualdad del triángulo, tenemos que $d(x, \tau y) \geq d(y, \tau y)-d(x, y) \geq r-1$. Luego $\min \mathcal{D}_{A}(Q, \tau Q) \geq r-1$. También observamos que $Q \neq \tau Q$ gracias a que, por definición, $r \geq 2$. Usando el teorema 2.1 concluimos que $d_{k(A)}(Q, \tau Q)=\min \mathcal{D}_{A}(Q, \tau Q)+1 \geq r$. Con lo que probamos que $k(\mathbb{A})$ es $r$-coafín y que $k$ es un operador en $\mathcal{G}^{(r)}$.

Es claro cómo extender los conceptos de $k$-carácter, $k$-convergencia, $k$-nulidad, etc. a las dos nuevas categorías. También es claro que $\mathbb{G}=\left(G, \tau_{G}\right)$ y $G$ tienen el mismo $k$-carácter.

Note que se sigue de inmediato que una gráfica coafín no puede ser $k$-nula.

El operador $k$ no es un funtor en ninguna de estas categorías. 


\section{Dominación en $\mathcal{G}, \mathcal{G}^{a}, \mathcal{G}^{(r)}$}

Sea $\mathcal{H}$ cualquiera de las categorías $\mathcal{G}, \mathcal{G}^{a}, \mathcal{G}^{(r)}$.

El concepto de dominación introducido por Neumann-Lara en $[55,57]$ ha probado ser de mucha utilidad en el estudio del $k$-carácter de las gráficas 2 -coafines. Lo transcribimos aquí:

Definición 4.2 Sean $\mathbb{A}, \mathbb{B} \in \mathcal{H}$. Decimos que $\mathbb{A}$ domina a $\mathbb{B}$ y lo escribimos $\mathbb{A} \succsim \mathbb{B}$, si existe $\mathbb{X} \in \mathcal{H}$ y existen dos morfismos $\alpha: \mathbb{X} \rightarrow \mathbb{A}$ y $\beta: \mathbb{X} \rightarrow \mathbb{B}$ tal que $\alpha$ es sobreyectivo y completo.

El concepto de dominación en $\mathcal{G}$ se define aquí por completez, aunque no es de utilidad puesto que $A \succsim B$ para cualesquiera $A, B \in \mathcal{G}$.

Los siguientes cuatro teoremas son probados por Neumann-Lara [55,57] para las categorías $\mathcal{G}, \mathcal{G}^{a}$ y $\mathcal{G}^{(2)}$. Técnicamente, no hay nada más que probar para $\mathcal{G}^{(r)}$ puesto que $\mathcal{G}^{(r)}$ es una subcategoría plena de $\mathcal{G}^{a}$, pero incluimos pruebas para la comodidad del lector.

Teorema 4.3 (Neumann-Lara $[55,57])$ La relación “ ” es un preorden en $\mathcal{H}$. Es decir, es una relación reflexiva y transitiva.

Prueba. La reflexividad es clara. Probaremos la transitividad. Sean $\mathbb{A}, \mathbb{B}, \mathbb{C} \in \mathcal{H}$ tal que $\mathbb{A} \succsim \mathbb{B} \succsim \mathbb{C}$. Sean $\mathbb{X}_{1}, \mathbb{X}_{2} \in \mathcal{H}$ y $\alpha_{1}: \mathbb{X}_{1} \rightarrow \mathbb{A}, \beta_{1}: \mathbb{X}_{1} \rightarrow \mathbb{B}, \alpha_{2}: \mathbb{X}_{2} \rightarrow \mathbb{B}$ $\beta_{2}: \mathbb{X}_{2} \rightarrow \mathbb{C}$ morfismos de $\mathcal{H}$ con $\alpha_{1}$ y $\alpha_{2}$ sobreyectivos y completos como se ilustra en el diagrama.

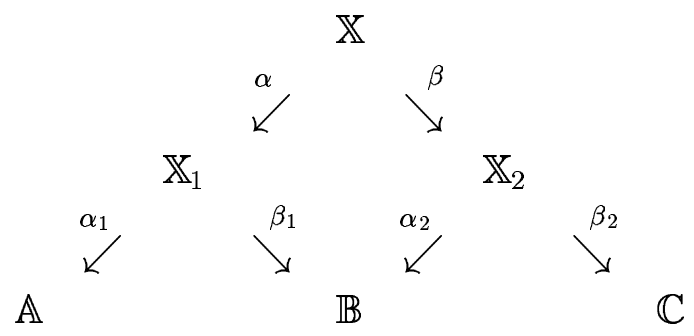


Sea $\mathbb{X}$ la subgráfica inducida en $\mathbb{X}_{1} \otimes \mathbb{X}_{2}$ por el conjunto de vértices $\left\{(u, v): \beta_{1}(u)=\right.$ $\left.\alpha_{2}(v)\right\}$ y sean $\alpha: \mathbb{X} \rightarrow \mathbb{X}_{1}$ y $\beta: \mathbb{X} \rightarrow \mathbb{X}_{2}$ las restricciones de las proyecciones de $\mathbb{X}_{1} \otimes \mathbb{X}_{2}$ en $\mathbb{X}_{1}$ y $\mathbb{X}_{2}$ respectivamente. Probaremos que $\alpha_{1} \circ \alpha \mathrm{y} \beta_{2} \circ \beta$ son morfismos con $\alpha_{1} \circ \alpha$ sobreyectivo y completo. Como $\alpha_{1}$ y $\beta_{2}$ ya son morfismos y $\alpha_{1}$ ya es sobreyectivo y completo, nos bastará probar que $\alpha$ y $\beta$ son morfismos y que $\alpha$ es sobreyectivo y completo.

Si $\mathcal{H}=\mathcal{G}, \alpha$ y $\beta$ son claramente morfismos y por tanto también lo son $\alpha_{1} \circ \alpha$ y $\beta_{2} \circ \beta$. Mostraremos que si $\mathcal{H}=\mathcal{G}^{a}, \alpha$ y $\beta$ son también morfismos:

$\operatorname{Si}(u, v) \in \mathbb{X}$ entonces $\left(\tau_{X_{1}}(u), \tau_{X_{2}}(v)\right) \in \mathbb{X}$ pues $\beta_{1}\left(\tau_{X_{1}}(u)\right)=\tau_{B}\left(\beta_{1}(u)\right)=\tau_{B}\left(\alpha_{2}(v)\right)=$ $\alpha_{2}\left(\tau_{X_{2}}(v)\right)$. La admisibilidad, se hereda de $\mathbb{X}_{1} \otimes \mathbb{X}_{2}$. Finalmente, en el caso $\mathcal{H}=\mathcal{G}^{(r)}$, $\alpha$ y $\beta$ son morfismos porque $\mathcal{G}^{(r)}$ es una subcategoría plena de $\mathcal{G}^{a}$.

Sólo resta probar que $\alpha$ es sobreyectivo y completo:

Sea $u \in X_{1}$, como $\alpha_{2}$ es sobreyectivo, existe una $v \in X_{2}$ tal que $\beta_{1}(u)=\alpha_{2}(v)$ y entonces $(u, v) \in X$. De modo que $\alpha$ es sobreyectivo.

Claramente, la preimagen de un vértice de $X_{1}$ es una completa. Si $\left\{u_{1}, u_{2}\right\}$ es una arista de $X_{1}$, y $\left(u_{1}, v_{1}\right),\left(u_{2}, v_{2}\right)$ son cualesquiera dos vértices en la preimagen de $u_{1} \mathrm{y}$ $u_{2}$ respectivamente, entonces, tenemos que $\alpha_{2}\left(v_{1}\right)=\beta_{1}\left(u_{1}\right)$ y $\alpha_{2}\left(v_{2}\right)=\beta_{1}\left(u_{2}\right)$ deben ser adyacentes o iguales en $B$, luego, por la completez de $\alpha_{2}$, tenemos que $v_{1}$ y $v_{2}$ son adyacentes o iguales en $X_{2}$. De modo que $\left(u_{1}, v_{1}\right)$ y $\left(u_{2}, v_{2}\right)$ son adyacentes en $X$. Se sigue que $\alpha$ es un morfismo completo.

Teorema 4.4 (Neumann-Lara $[55,57])$ Para toda $\mathbb{A}, \mathbb{B} \in \mathcal{H}, \mathbb{A} \succsim \mathbb{B}$ implica $k(\mathbb{A}) \succsim$ $k(\mathbb{B})$.

Prueba. Por hipótesis, existe $\mathbb{X} \in \mathcal{H}$ y dos morfismos $\alpha: \mathbb{X} \rightarrow \mathbb{A}, \beta: \mathbb{X} \rightarrow \mathbb{B}$, con $\alpha$ sobreyectivo y completo.

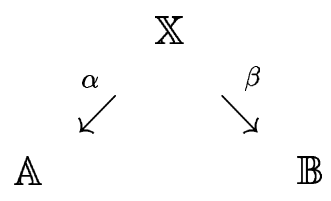

Sea $\mathbb{X}^{\prime}$ la subgráfica inducida en $k(\mathbb{X}) \otimes k(\mathbb{B})$ por el conjunto $\{(P, Q): \beta(P) \subseteq Q\}$ y sean $\alpha^{\prime}: \mathbb{X}^{\prime} \rightarrow k(\mathbb{X})$ y $\beta^{\prime}: \mathbb{X}^{\prime} \rightarrow k(\mathbb{B})$ las restricciones de las proyecciones de 
$k(\mathbb{X}) \otimes k(\mathbb{B})$ en $k(\mathbb{X})$ y $k(\mathbb{B})$ respectivamente.

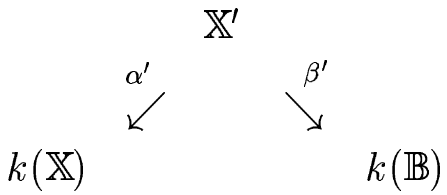

Probaremos que $\alpha^{\prime}$ y $\beta^{\prime}$ son morfismos, con $\alpha^{\prime}$ sobreyectivo y completo. También probaremos que $k(\mathbb{X}) \cong k(\mathbb{A})$.

Si $\mathcal{H}=\mathcal{G}, \alpha^{\prime}$ y $\beta^{\prime}$ son claramente morfismos. En el caso $\mathcal{H}=\mathcal{G}^{a}$, sólo hay que probar que $\mathbb{X}^{\prime}$ es una subgráfica $\left(\tau\right.$-invariante) de $k(\mathbb{X}) \otimes k(\mathbb{B}):$ Si $(P, Q) \in \mathbb{X}^{\prime}$ tenemos $\tau(P, Q)=\left(\tau_{X}(P), \tau_{B}(Q)\right)$, pero $\beta\left(\tau_{X}(P)\right)=\tau_{B}(\beta(P)) \subseteq \tau_{B}(Q)$. Así que $\mathbb{X}^{\prime}$ es una subgráfica de $k(\mathbb{X}) \otimes k(\mathbb{B})$ (en $\mathcal{G}^{a}$ ) y tanto $\alpha^{\prime}$ como $\beta^{\prime}$ son morfismos admisibles. Finalmente, recuerde que $\mathcal{G}^{(r)}$ es una subcategoría plena de $\mathcal{G}^{a}$.

Es una verificación rutinaria el que $\alpha^{\prime}$ es sobreyectivo y completo.

Finalmente, observamos que si $Q \in k(A), \alpha^{-1}(Q)$ es un clan de $X$ y que si $P \in k(X)$, $\alpha(P)$ es un clan de $A$. Las funciones así descritas, son inversas una de la otra y claramente son admisibles, de modo que $k(\mathbb{A}) \cong k(\mathbb{X})$.

Teorema 4.5 (Neumann-Lara [55,57]) Para toda $\mathbb{A}, \mathbb{B} \in \mathcal{H}$, Si $f: \mathbb{A} \rightarrow \mathbb{B}$ es un morfismo, entonces $\mathbb{A} \succsim \mathbb{B}$.

Prueba. Obvio.

Teorema 4.6 (Neumann-Lara [55]) Para toda $\mathbb{A} \in \mathcal{H}, \mathbb{A} \succsim k^{2}(\mathbb{A})$.

Prueba. Sea $\mathbb{X}$ la subgráfica de $\mathbb{A} \otimes k^{2}(\mathbb{A})$ inducida por $\{(x, P): *(x) \subseteq P\}$ con $\alpha$ y $\beta$ las restricciones de siempre $\mathrm{y} *(x)=\{Q \in k(A): x \in Q\}$. Usando las mismas ideas de las demostraciones anteriores, el resto es ya sólo una verificación de rutina. 


\section{Sumas $r$-coafines}

La suma de Zykov (unión disjunta de dos gráficas más todas las posibles aristas entre ellas) juega un papel central en la teoría de gráficas expansivas de Neumann-Lara, y su adecuada generalización es la clave para nuestra teoría de gráficas divergentes en sumandos:

Definición 4.7 Sea $\mathbb{G} \in \mathcal{G}^{(r)}, \mathbb{A}$ una subgráfica de $\mathbb{G}$ y sean $\mathbb{A}_{1}, \mathbb{A}_{2}, \ldots, \mathbb{A}_{n}$ subgráficas de $\mathbb{A}$. Decimos que $\mathbb{A}$ es suma $r$-coafín de $\mathbb{A}_{1}, \mathbb{A}_{2}, \ldots, \mathbb{A}_{n}$ con respecto a $\mathbb{G}$ y lo escribimos $\mathbb{A}=\bigoplus_{\mathbb{G}} \mathbb{A}_{i}$, si se cumplen las siguientes condiciones:

1. $\left\{V\left(\mathbb{A}_{1}\right), \ldots, V\left(\mathbb{A}_{n}\right)\right\}$ es una partición de $V(\mathbb{A})$.

2. $d_{\mathbb{G}}(x, y) \leq r-1$ para toda $x \in V\left(\mathbb{A}_{i}\right)$ y $y \in V\left(\mathbb{A}_{j}\right)$ siempre que $i \neq j$.

En algunos casos también escribiremos: $\mathbb{A}=\mathbb{A}_{1} \bigoplus_{\mathbb{G}} \mathbb{A}_{2} \bigoplus_{\mathbb{G}} \cdots \bigoplus_{\mathbb{G}} \mathbb{A}_{n}$.

Definición 4.8 Definimos el rango de $\mathbb{G}$, que escribimos como rango $(\mathbb{G})$, como el máximo número $n$ tal que existe una subgráfica $\mathbb{A}$ de $\mathbb{G}$ y $n$ subgráficas $\mathbb{A}_{1}, \ldots, \mathbb{A}_{n}$ de $\mathbb{G}$ tal que $\mathbb{A}=\bigoplus_{\mathbb{G}} \mathbb{A}_{i}$.

Teorema 4.9 Si $\mathbb{G}, \mathbb{G}^{\prime} \in \mathcal{G}^{(r)}, \mathbb{A}=\mathbb{A}_{1} \bigoplus_{\mathbb{G}} \mathbb{A}_{2} \bigoplus_{\mathbb{G}} \cdots \bigoplus_{\mathbb{G}} \mathbb{A}_{n}$ es una subgráfica de $\mathbb{G}$ y $f: \mathbb{G} \rightarrow \mathbb{G}^{\prime}$ es un morfismo, entonces $f(\mathbb{A})=f\left(\mathbb{A}_{1}\right) \bigoplus_{\mathbb{G}^{\prime}} f\left(\mathbb{A}_{2}\right) \bigoplus_{\mathbb{G}^{\prime}} \cdots \bigoplus_{\mathbb{G}^{\prime}} f\left(\mathbb{A}_{n}\right)$.

Prueba. Sea $\mathbb{G}^{\prime}=\left(G^{\prime}, \tau^{\prime}\right)$. En primer lugar notamos que $f(\mathbb{A})$ es una subgráfica de $\mathbb{G}^{\prime}$ y que cada $f\left(\mathbb{A}_{i}\right)$ es subgráfica de $f(\mathbb{A})$. Claramente $V(f(\mathbb{A}))=\cup_{i=1}^{n} V\left(f\left(\mathbb{A}_{i}\right)\right)$ y $V\left(f\left(\mathbb{A}_{i}\right)\right) \neq \varnothing$ para toda $i$. Como la imagen de cualquier trayectoria bajo un morfismo es un camino, también tenemos que $d_{\mathbb{G}}(x, y) \leq r-1$ para toda $x \in$ $V\left(f\left(\mathbb{A}_{i}\right)\right)$ y $y \in V\left(f\left(\mathbb{A}_{j}\right)\right)$ siempre que $i \neq j$. Sólo resta probar que $V\left(f\left(\mathbb{A}_{i}\right)\right) \cap$ $V\left(f\left(\mathbb{A}_{j}\right)\right)=\varnothing$ para toda $i \neq j$.

Suponga que $i \neq j$ y $x \in V\left(f\left(\mathbb{A}_{i}\right)\right) \cap V\left(f\left(\mathbb{A}_{j}\right)\right)$. Como $\mathbb{G}^{\prime}$ es $r$-coafín tenemos que $d_{\mathbb{G}^{\prime}}\left(x, \tau^{\prime}(x)\right) \geq r$, pero por otro lado, $x \in V\left(f\left(\mathbb{A}_{i}\right)\right)$ y $\tau^{\prime}(x) \in V\left(f\left(\mathbb{A}_{j}\right)\right)$ implica que $d_{\mathbb{G}^{\prime}}\left(x, \tau^{\prime}(x)\right) \leq r-1$. Contradicción. 
Teorema 4.10 Si $\mathbb{G}, \mathbb{G}^{\prime} \in \mathcal{G}^{(r)}, \mathbb{A}=\mathbb{A}_{1} \bigoplus_{\mathbb{G}} \mathbb{A}_{2} \bigoplus_{\mathbb{G}} \cdots \bigoplus_{\mathbb{G}} \mathbb{A}_{n}$ es una subgráfica de $\mathbb{G}$ y $f: \mathbb{G}^{\prime} \rightarrow \mathbb{G}$ es un morfismo sobreyectivo y completo, entonces $f^{-1}(\mathbb{A})=$ $f^{-1}\left(\mathbb{A}_{1}\right) \bigoplus_{\mathbb{G}^{\prime}} f^{-1}\left(\mathbb{A}_{2}\right) \bigoplus_{\mathbb{G}^{\prime}} \cdots \bigoplus_{\mathbb{G}^{\prime}} f^{-1}\left(\mathbb{A}_{n}\right)$.

Prueba. Observamos que $f^{-1}(\mathbb{A})$ es una subgráfica de $\mathbb{G}^{\prime}$ y que cada $f^{-1}\left(\mathbb{A}_{i}\right)$ es subgráfica de $f^{-1}(\mathbb{A})$. Claramente $V\left(f^{-1}(\mathbb{A})\right)=\cup_{i=1}^{n} V\left(f^{-1}\left(\mathbb{A}_{i}\right)\right)$. Si $x \in$ $V\left(f^{-1}\left(\mathbb{A}_{i}\right)\right) \cap V\left(f^{-1}\left(\mathbb{A}_{j}\right)\right)$ para algunas $i \neq j$, tendríamos que $f(x) \in V\left(\mathbb{A}_{i}\right) \cap V\left(\mathbb{A}_{j}\right)$ contrario a la hipótesis de que $\mathbb{A}=\mathbb{A}_{1} \bigoplus_{\mathbb{G}} \mathbb{A}_{2} \bigoplus_{\mathbb{G}} \cdots \bigoplus_{\mathbb{G}} \mathbb{A}_{n}$. Gracias a que $f$ es sobreyectivo, tenemos que $V\left(f^{-1}\left(\mathbb{A}_{i}\right)\right) \neq \varnothing$ para toda $i$. Como $f$ es completo, tenemos que $d_{\mathbb{G}^{\prime}}(x, y) \leq r-1$ para toda $x \in V\left(f^{-1}\left(\mathbb{A}_{i}\right)\right)$ y $y \in V\left(f^{-1}\left(\mathbb{A}_{j}\right)\right)$ siempre que $i \neq j$.

\section{Gráficas divergentes en sumandos}

El siguiente teorema relaciona el rango con la dominación ${ }^{1}$ :

Teorema 4.11 Si $\mathbb{G} \succsim \mathbb{G}^{\prime}$ en $\mathcal{G}^{(r)}$ entonces rango $(\mathbb{G}) \leq$ rango $\left(\mathbb{G}^{\prime}\right)$. En particular, $\operatorname{rango}(\mathbb{G}) \leq \operatorname{rango}\left(k^{2}(\mathbb{G})\right)$ para toda $\mathbb{G} \in \mathcal{G}^{(r)}$.

Prueba. Sea $\mathbb{X}$ una gráfica $r$-coafín tal que existen dos morfismos $\left(\right.$ en $\mathcal{G}^{(r)}$ ) $\alpha: \mathbb{X} \rightarrow \mathbb{G}$ y $\beta: \mathbb{X} \rightarrow \mathbb{G}^{\prime}$ con $\alpha$ sobreyectivo y completo. Sea $n=\operatorname{rango}(\mathbb{G})$ y $\mathbb{A}=\mathbb{A}_{1} \bigoplus_{\mathbb{G}} \mathbb{A}_{2} \bigoplus_{\mathbb{G}} \cdots \bigoplus_{\mathbb{G}} \mathbb{A}_{n}$. Por el teorema anterior,

$$
\alpha^{-1}(\mathbb{A})=\alpha^{-1}\left(\mathbb{A}_{1}\right) \bigoplus_{\mathbb{X}} \alpha^{-1}\left(\mathbb{A}_{2}\right) \bigoplus_{\mathbb{X}} \cdots \bigoplus_{\mathbb{X}} \alpha^{-1}\left(\mathbb{A}_{n}\right)
$$

y luego, por el teorema 4.9, tenemos

$$
\beta\left(\alpha^{-1}(\mathbb{A})\right)=\beta\left(\alpha^{-1}\left(\mathbb{A}_{1}\right)\right) \bigoplus_{\mathbb{G}^{\prime}} \beta\left(\alpha^{-1}\left(\mathbb{A}_{2}\right)\right) \bigoplus_{\mathbb{G}^{\prime}} \cdots \bigoplus_{\mathbb{G}^{\prime}} \beta\left(\alpha^{-1}\left(\mathbb{A}_{n}\right)\right) .
$$

De modo que rango $(\mathbb{G}) \leq \operatorname{rango}\left(\mathbb{G}^{\prime}\right)$.

Por el teorema 4.6, tenemos rango $(\mathbb{G}) \leq \operatorname{rango}\left(k^{2}(\mathbb{G})\right)$.

Tenemos ahora la definición central de este capítulo:

\footnotetext{
${ }^{1} \mathrm{El}$ teorema 4.11 parece indicarnos que deberíamos escribir $\mathbb{G} \precsim \mathbb{G}^{\prime}$ en lugar de $\mathbb{G} \succsim \mathbb{G}^{\prime}$. Usamos esta notación para ser consistentes con [55].
} 
Definición 4.12 Decimos que $\mathbb{G} \in \mathcal{G}^{(r)}$ es divergente en sumandos si el conjunto $\left\{\operatorname{rango}\left(k^{n}(\mathbb{G})\right): n \in \mathbb{N}\right\}$ no está acotado.

Claramente, $\mathbb{G}$ es divergente en sumandos si y sólo si $k^{n}(\mathbb{G})$ es divergente en sumandos para cualquier $n$. La razón por la que nos interesa la divergencia en sumandos es que ésta es una forma de clan divergencia:

Teorema 4.13 Si $\mathbb{G} \in \mathcal{G}^{(r)}$ es divergente en sumandos, entonces es $k$-divergente.

Prueba. Claramente rango $\left(k^{n}(\mathbb{G})\right) \leq\left|V\left(k^{n}(\mathbb{G})\right)\right|$.

\section{El teorema de la divergencia en sumandos}

Llegamos así al teorema principal del capítulo:

Teorema 4.14 (Teorema de la divergencia en sumandos) Si $\mathbb{G}, \mathbb{G}^{\prime} \in \mathcal{G}^{(r)}$ con $\mathbb{G}$ divergente en sumandos y $\mathbb{G} \succsim \mathbb{G}^{\prime}$, entonces $\mathbb{G}^{\prime}$ también es divergente en sumandos.

Prueba. Inmediato de los teoremas 4.11 y 4.4 .

Encontramos que el producto fuerte tiene nuevas e interesantes propiedades en relación a las gráficas divergentes en sumandos:

Teorema 4.15 Si $\mathbb{A}, \mathbb{B} \in \mathcal{G}^{(r)}$ y $\mathbb{A} \otimes \mathbb{B}$ es divergente en sumandos, entonces $\mathbb{A} y \mathbb{B}$ también lo son.

Prueba. Como las proyecciones son morfismos en la categoría, tenemos $\mathbb{A} \otimes \mathbb{B} \succsim \mathbb{A}$ y $\mathbb{A} \otimes \mathbb{B} \succsim \mathbb{B}$.

Teorema 4.16 $\operatorname{rango}(\mathbb{X} \otimes \mathbb{Y})=\min \{\operatorname{rango}(\mathbb{X}), \operatorname{rango}(\mathbb{Y})\}$ 
Prueba. $\quad$ Como $\mathbb{X} \otimes \mathbb{Y} \succsim \mathbb{X}$ y $\mathbb{X} \otimes \mathbb{Y} \succsim$, por el teorema 4.11 tenemos que: $\operatorname{rango}(\mathbb{X} \otimes \mathbb{Y}) \leq \min \{\operatorname{rango}(\mathbb{X}), \operatorname{rango}(\mathbb{Y})\}$

Sean $\mathbb{M}=\mathbb{M}_{1} \bigoplus_{\mathbb{X}} \mathbb{M}_{2} \bigoplus_{\mathbb{X}} \cdots \bigoplus_{\mathbb{X}} \mathbb{M}_{m}$ y $\mathbb{N}=\mathbb{N}_{1} \bigoplus_{\mathbb{Y}} \mathbb{N}_{2} \bigoplus_{\mathbb{Y}} \cdots \bigoplus_{\mathbb{Y}} \mathbb{N}_{n}$ con $m=$ rango $(\mathbb{X})$ y $n=\operatorname{rango}(\mathbb{Y})$. Sin pérdida de generalidad, supongamos que $m \leq n$. Probaremos que $\mathbb{P}=\left(\mathbb{M}_{1} \otimes \mathbb{N}_{1}\right) \bigoplus_{\mathbb{X} \otimes \mathbb{Y}}\left(\mathbb{M}_{2} \otimes \mathbb{N}_{2}\right) \bigoplus_{\mathbb{X} \otimes \mathbb{Y}} \cdots \bigoplus_{\mathbb{X} \otimes \mathbb{Y}}\left(\mathbb{M}_{m} \otimes \mathbb{N}_{m}\right)$. Es claro que cada $\mathbb{M}_{i} \otimes \mathbb{N}_{i}$ es una subgráfica de $\mathbb{X} \otimes \mathbb{Y}$ y que $\left\{V\left(\mathbb{M}_{i} \otimes \mathbb{N}_{i}\right)\right\}$ es una partición de $V(\mathbb{P})$. En el caso en que $u \in \mathbb{M}_{i} \otimes \mathbb{N}_{i}$ y $v \in \mathbb{M}_{j} \otimes \mathbb{N}_{j}$ con $i \neq j$, para probar que $d_{\mathbb{X} \otimes \mathbb{Y}}(u, v) \leq r-1$ basta con recordar que si $u=\left(u_{1}, u_{2}\right)$ y $v=\left(v_{1}, v_{2}\right)$ entonces $d_{\mathbb{X} \mathbb{Y}}(u, v)=\max \left\{d_{\mathbb{X}}\left(u_{1}, v_{1}\right), d_{\mathbb{Y}}\left(u_{2}, v_{2}\right)\right\}$. Concluimos que $\operatorname{rango}(\mathbb{X} \otimes \mathbb{Y}) \geq \min \{\operatorname{rango}(\mathbb{X}), \operatorname{rango}(\mathbb{Y})\}$.

Teorema 4.17 Si $\lim _{n \rightarrow \infty} \operatorname{rango}\left(k^{n}(\mathbb{A})\right)=\infty=\lim _{n \rightarrow \infty} \operatorname{rango}\left(k^{n}(\mathbb{B})\right)$, entonces $\mathbb{A} \otimes \mathbb{B}$ es divergente en sumandos.

Prueba. Es fácil verificar que el teorema 1.1 vale para gráficas automorfas, de modo que $k^{n}(\mathbb{A} \otimes \mathbb{B})=k^{n}(\mathbb{A}) \otimes k^{n}(\mathbb{B})$ para toda $n$. Terminamos usando el teorema anterior.

Observamos que el teorema anterior no es el recíproco del teorema 4.15.

Problema $10 \dot{A} \otimes \mathbb{B}$ es divergente en sumandos siempre que $\mathbb{A} y \mathbb{B}$ lo son?

Es fácil probar usando el teorema 4.6 que el siguiente problema es equivalente al anterior.

Problema 11 ¿A es divergente en sumandos ssi $\lim _{n \rightarrow \infty} \operatorname{rango}\left(k^{n}(\mathbb{A})\right)=\infty$ ?

\section{Relojes divergentes en sumandos}

En las secciones precedentes, hemos desarrollado técnicas útiles para concluir la divergencia en sumandos (y la clan divergencia) de una gráfica $\mathbb{A}$ siempre que haya otra gráfica $\mathbb{B}$ divergente en sumandos que la domine. Sin embargo, nuestra teoría es vacía mientras no tengamos algún ejemplo de gráfica divergente en sumandos. 
En el caso $r=2$ las gráficas divergentes en sumandos son precisamente las gráficas expansivas estudiadas por Neumann-Lara [55,57]. Para $r \geq 3$, Encontraremos los preciados ejemplos entre los relojes estudiados en el capítulo 3.

Diremos que un reloj $r$-coafín es una gráfica $r$-coafín $\mathbb{G}=(G, \tau)$ donde $G$ es un reloj. Retomamos los ejemplos 3.15 y 3.16, para verlos ahora como relojes $r$-coafines.

Ejemplo 4.18 Definimos al reloj $(m+1)$-coafin $\mathbb{T}_{2 m}^{n}$, por $\mathbb{T}_{2 m}^{n}=\left(T_{2 m}^{n}, \tau_{2 m}^{n}\right)$ donde $T_{2 m}^{n}$ es el reloj del ejemplo 3.15 y el automorfismo $\tau_{2 m}^{n}$ está dado por:

$$
\begin{aligned}
\tau_{2 m}^{n}\left(x_{0}^{i}\right) & =x_{1}^{i+m} \\
\tau_{2 m}^{n}\left(x_{1}^{i}\right) & =x_{0}^{i+m} \\
\tau_{2 m}^{n}\left(x_{j}^{i}\right) & =x_{j}^{i+m} \text { siempre que } j \neq 0,1 .
\end{aligned}
$$

Observamos entonces que $k\left(\mathbb{T}_{2 m}^{n}\right)=\left(k\left(T_{2 m}^{n}\right),\left(\tau_{2 m}^{n}\right)_{k\left(T_{2 m}^{n}\right)}\right) \cong \mathbb{T}_{2 m}^{n+1}$.

Teorema $4.19 \mathbb{T}_{2 m}^{n}$ es una gráfica $(m+1)$-coafín divergente en sumandos.

\section{Prueba.}

Tomemos $x_{j}^{i}, x_{j^{\prime}}^{i^{\prime}} \in V\left(T_{2 m}^{n}\right)$. Definimos la distancia unidireccional $\vec{d}\left(x_{j}^{i}, x_{j^{\prime}}^{i^{\prime}}\right)$ como:

$$
\vec{d}\left(x_{j}^{i}, x_{j^{\prime}}^{i^{\prime}}\right)=\left\{\begin{array}{l}
0 \text { si } i=i^{\prime} \text { y } j=j^{\prime} \\
1 \text { si } i=i^{\prime} \text { y } j \neq j^{\prime} \\
\left|i^{\prime}-i\right| \text { si } j=j^{\prime} \in\{0,1\} \\
\left|i^{\prime}-i\right| \text { si } j^{\prime} \in\{0,1\} \text { y } j \geq 2 \\
\left|i^{\prime}-i\right| \text { si }\left(j-j^{\prime}\right) \geq\left|i^{\prime}-i\right| \\
\left|i^{\prime}-i\right|+1 \text { en caso contrario. }
\end{array}\right.
$$

Una verificación de rutina, nos dice que $\vec{d}\left(x_{j}^{i}, x_{j^{\prime}}^{i^{\prime}}\right)$ es la longitud de la trayectoria más corta que une a $x_{j}^{i}$ con $x_{j^{\prime}}^{i^{\prime}}$ usando solamente vértices que estén en los segmentos $G_{i}, G_{i+1}, \ldots, G_{i^{\prime}}$. Luego es claro que la distancia en $T_{2 m}^{n}$ de $x_{j}^{i}$ a $x_{j^{\prime}}^{i^{\prime}}$ es $d\left(x_{j}^{i}, x_{j^{\prime}}^{i^{\prime}}\right)=$ $\min \left\{\vec{d}\left(x_{j}^{i}, x_{j^{\prime}}^{i^{\prime}}\right), \vec{d}\left(x_{j^{\prime}}^{i^{\prime}}, x_{j}^{i}\right)\right\}$. Así tenemos que $\mathbb{T}_{2 m}^{n}$ es $(m+1)$-coafín.

Tomemos ahora $A_{t}=\left\{x_{2+m t}^{0}, x_{2+m t}^{m}\right\}$, para $t=0, \ldots,\left\lfloor\frac{n}{m}\right\rfloor$. Es fácil notar que cada $A_{t}$ es $\tau_{2 m}^{n}$-invariante y que el conjunto de distancias de $A_{t}$ a $A_{t^{\prime}}$ es $D\left(A_{t}, A_{t^{\prime}}\right)=\{1, m\}$ 
siempre que $t \neq t^{\prime}$. Luego, tomando $\mathbb{A}_{t}=\mathbb{T}_{2 m}^{n}\left[A_{t}\right]$ y $\mathbb{A}=\mathbb{T}_{2 m}^{n}\left[A_{0} \cup A_{1} \cup \cdots \cup A_{\left\lfloor\frac{n}{m}\right\rfloor}\right]$ tenemos que $\mathbb{A}=\bigoplus_{\mathbb{T}_{2 m}^{n}} \mathbb{A}_{t}$ y entonces rango $\left(\mathbb{T}_{2 m}^{n}\right) \geq\left\lfloor\frac{n}{m}\right\rfloor$, y así hemos probado que $\mathbb{T}_{2 m}^{n}$ es divergente en sumandos.

Ejemplo 4.20 Definimos al reloj $(m+1)$-coafin $\mathbb{H}_{m}^{n}$, por $\mathbb{H}_{m}^{n}=\left(H_{m}^{n}, \eta_{m}^{n}\right)$ donde $H_{m}^{n}$ es el reloj del ejemplo 3.16 (figura 3.4) y el automorfismo $\eta_{m}^{n}$ está dado por:

$$
\begin{aligned}
\eta_{m}^{n}\left(x_{0}^{i}\right) & =x_{1}^{i+m} \\
\eta_{m}^{n}\left(x_{1}^{i}\right) & =x_{0}^{i+m} \\
\eta_{m}^{n}\left(x_{j}^{i}\right) & =x_{j}^{i+m} \text { siempre que } j \neq 0,1 .
\end{aligned}
$$

Observamos entonces que $k\left(\mathbb{H}_{m}^{n}\right)=\left(k\left(H_{m}^{n}\right),\left(\eta_{m}^{n}\right)_{k\left(H_{m}^{n}\right)}\right) \cong \mathbb{H}_{m}^{n+1}$.

Teorema $4.21 \mathbb{H}_{m}^{n}$ es una gráfica $(m+1)$-coafín divergente en sumandos.

Prueba. La prueba de que es $(m+1)$ - coafín es muy semejante a la del teorema anterior. $\mathbb{H}_{m}^{n}$ es divergente en sumandos porque hay un morfismo de gráficas $(m+1)$-coafines $\varphi: \mathbb{T}_{2 m}^{0} \rightarrow \mathbb{H}_{m}^{0}$ dado por la función identidad en en los conjuntos de vértices, es decir $\varphi$ es la función que manda al vértice $x_{j}^{i}$ de $\mathbb{T}_{2 m}^{0}$ en el vértice $x_{j}^{i}$ de $\mathbb{H}_{m}^{0}$. Terminamos usando los teoremas 4.5 y 4.14

Tenemos aquí un comportamiento muy interesante con este par de gráficas coafines: Aunque rango $\left(k^{n}\left(\mathbb{T}_{2 m}^{0}\right)\right) \leq \operatorname{rango}\left(k^{n}\left(\mathbb{H}_{m}^{0}\right)\right)$, pasa lo contrario con los órdenes de las gráficas: $\left|V\left(k^{n}\left(\mathbb{T}_{2 m}^{0}\right)\right)\right|=6 m+2 m n>6 m+2 n=\left|V\left(k^{n}\left(\mathbb{H}_{m}^{0}\right)\right)\right|$ para $n \geq 1$.

Además, los rangos de ambas gráficas son periódicamente iguales y distintos, pues se puede probar que rango $\left(k^{n}\left(\mathbb{T}_{2 m}^{0}\right)\right)=3 m+m\left\lfloor\frac{n}{m}\right\rfloor \mathrm{y} \operatorname{rango}\left(k^{n}\left(\mathbb{H}_{m}^{0}\right)\right)=3 m+n$.

Los octaedros y las gráficas de los ejemplos 4.18 y 4.20 son todos los ejemplos primitivos de gráficas divergentes en sumandos que conozco. Son pocos y sería bueno conocer más. Sin embargo veremos que, usando el teorema de la divergencia en sumandos, estos pocos ejemplos primitivos son útiles para probar la clan divergencia de una amplia gama de gráficas. 


\section{El icosaedro}

El problema del $k$-carácter del icosaedro fue (implícitamente) planteado en [57] y citado en $[40,45,58]$. Recientemente resolví el problema [61] usando gráficas absolutamente saturadas: una generalización parcial de la teoría de gráficas expansivas de Neumann-Lara [55,57]. Como la idea de gráfica divergente en sumandos es una generalización común a ambas teorías, también podemos probar la divergencia del icosaedro con estas técnicas:

Teorema 4.22 El icosaedro es divergente en sumandos.

Prueba. El automorfismo antípoda del icosaedro es 3-coafín. Sea II la gráfica automorfa que consta del icosaedro y el automorfismo antípoda. Hay un morfismo de gráficas 3 -coafines $\varphi: \mathbb{H}_{2}^{0} \rightarrow k(\mathbb{I})$ :

En la figura 4.1, después de identificar los vértices con etiqueta 1 y los vértices con etiqueta 9 (respectivamente), se muestra un dibujo del icosaedro. Los clanes del icosaedro son todos los triángulos y los triángulos sombreados son la imagen de $\mathbb{H}_{2}^{0}$ bajo $\varphi$. Es claro que $\mathbb{H}_{2}^{0}$ es isomorfa a su imagen bajo $\varphi$ y que $\varphi$ es admisible.

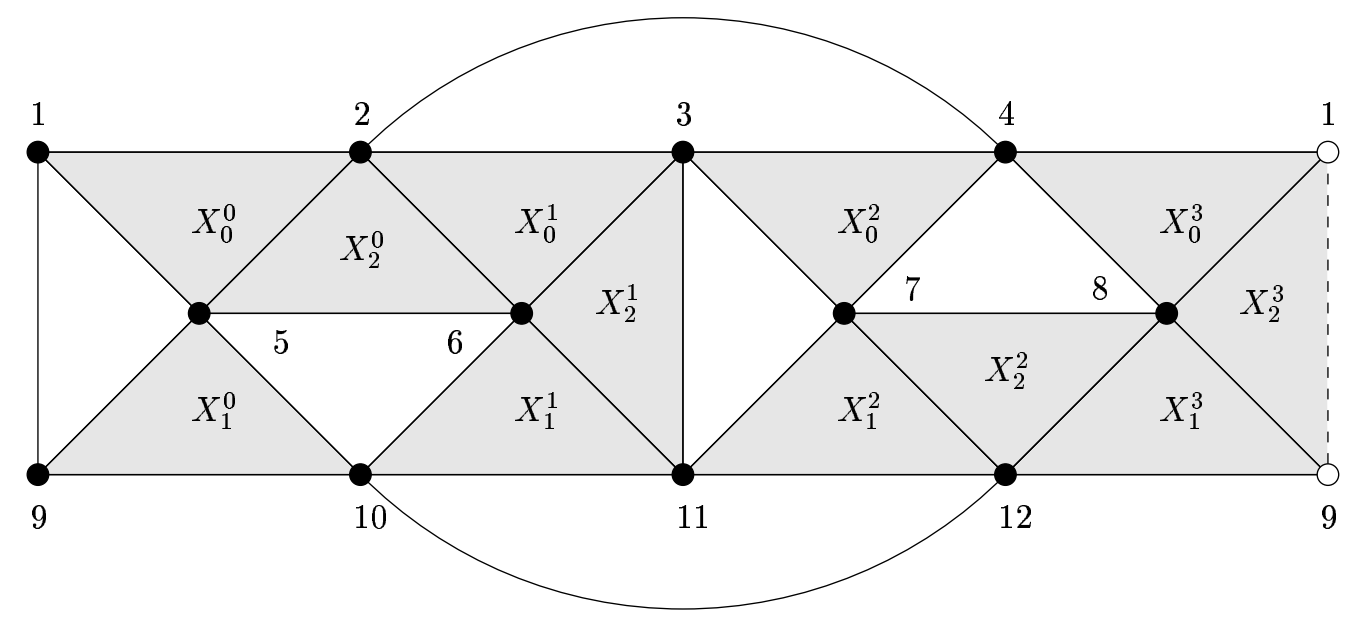

Figura 4.1: Icosaedro con una inmersión de $\mathbb{H}_{2}^{0}$ en $k(\mathbb{I})$

Cabe mencionar que el icosaedro no es expansivo (que de hecho es lo que se preguntaba en [57]). De modo que este es nuestro primer ejemplo de una gráfica cuya clan divergencia no se puede probar con las técnicas de gráficas expansivas pero sí con 
las de las gráficas divergentes en sumandos. Veremos a continuación, estas mismas ideas sirven para dar triangulaciones $k$-divergentes para cualquier superficie.

\section{Superficies $k$-divergentes}

En esta sección necesitaremos una cierta triangulación 4-coafín de la esfera. Aunque las triangulaciones 4-coafines como la que necesitamos son fáciles de construir, preferimos usar una triangulación 5-coafín (recuerde que toda gráfica $r$-coafín es también $(r-1)$-coafín), porque así tenemos una gráfica más simétrica y más bonita. La triangulación que escogemos pues es el dodecaedro estelado que se construye empezando con el dodecaedro, agregando luego un vértice a cada cara y haciendo adyacente cada uno de estos vértices a todos los vértices de su cara. Esta triangulación es de Whitney por el teorema 1.3.

Sea $\mathbb{S}$ la gráfica automorfa que consta del dodecaedro estelado y el automorfismo antípoda. En la figura 4.2, después de identificar los vértices con la etiquetas iguales, observamos un dibujo del dodecaedro estelado. Los vértices que conforman el dodecaedro original aparecen más grandes. La coafinación antípoda la obtenemos del dibujo, al hacer un desplazamiento a la derecha de longitud igual a la mitad de la del dibujo y luego reflejando por el eje de simetría horizontal.

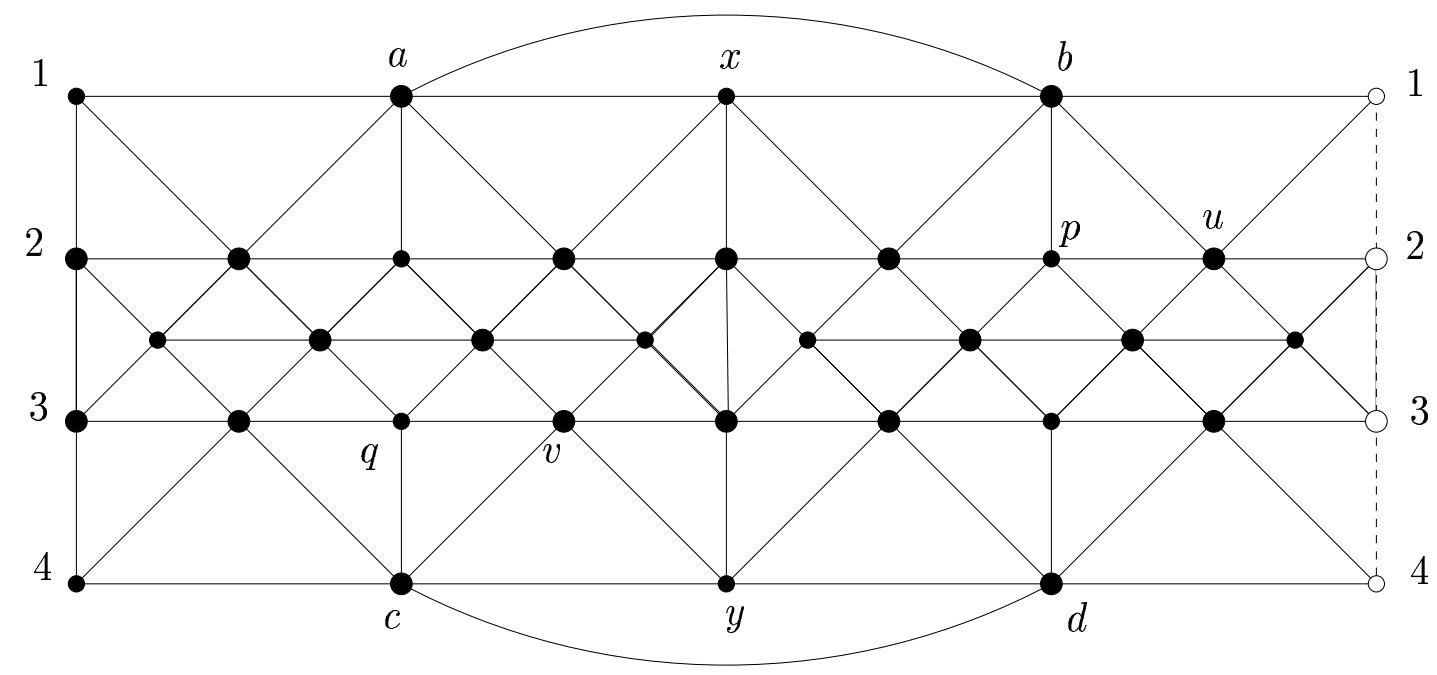

Figura 4.2: Dodecaedro estelado $\mathbb{S}$ : triangulación 5-coafín de una esfera

Teorema 4.23 El dodecaedro estelado $\mathbb{S}$ es 5 -coafín divergente en sumandos. 
Prueba. Observe la figura 4.3

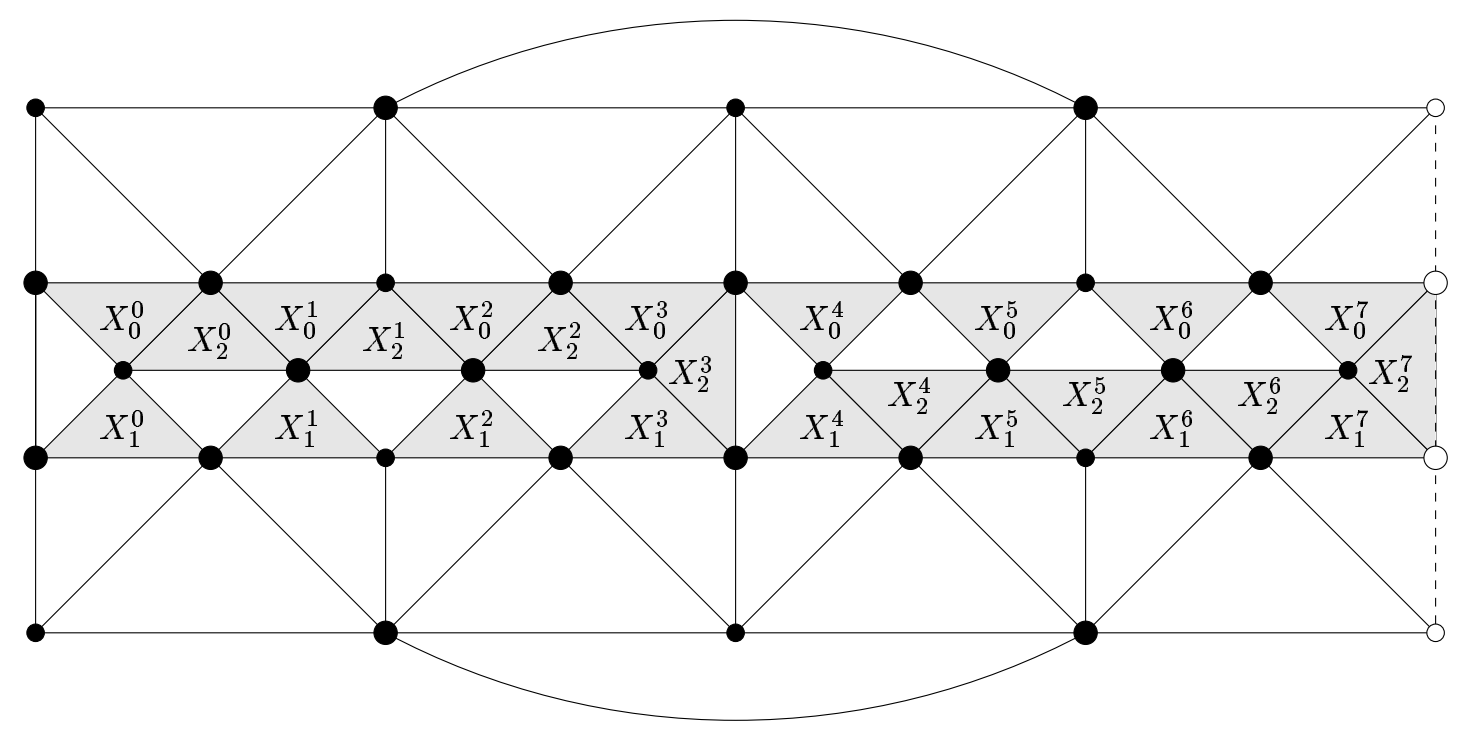

Figura 4.3: Dodecaedro estelado con una inmersión de $\mathbb{H}_{4}^{0}$ en $k(\mathbb{S})$

Sabemos pues que la esfera admite triangulaciones divergentes en sumandos. Se sabe también que el toro admite triangulaciones $k$-divergentes $[39,40]$. Veremos a continuación que el toro también admite triangulaciones divergentes en sumandos.

Tomemos $\mathbb{S}$ y eliminemos las aristas $\{a, b\}$ y $\{c, d\}$ para obtener la gráfica 5 -coafín $\mathbb{S}_{1}$. Observe que $\mathbb{S}_{1}$ todavía es divergente en sumandos. Sea $\mathbb{S}_{2}$ una copia disjunta de $\mathbb{S}_{1}$ y sean $\left\{1^{\prime}, a^{\prime}, x^{\prime}, b^{\prime}, 4^{\prime}, c^{\prime}, y^{\prime}, d^{\prime}\right\}$ los vértices correspondientes en $\mathbb{S}_{2}$ a $\{1, a, x, b, 4, c, y, d\}$. Definimos $\mathbb{T}$ como la gráfica que se obtiene de la unión disjunta de $\mathbb{S}_{1}$ y $\mathbb{S}_{2}$ al hacer las identificaciones de vértices: $1=1^{\prime}, a=a^{\prime}, x=x^{\prime}, b=b^{\prime}, 4=4^{\prime}, c=c^{\prime}$, $y=y^{\prime}$ y $d=d^{\prime}$. Observamos que $\mathbb{T}$ tiene una 5 -coafinación cuyas restricciones a las subgráficas $\mathbb{S}_{1}$ y $\mathbb{S}_{2}$ son las coafinaciones originales de $\mathbb{S}_{1}$ y $\mathbb{S}_{2}$ respectivamente. Es inmediato el teorema siguiente:

Teorema $4.24 \mathbb{T}$ es una triangulación del toro 5 -coafín divergente en sumandos.

Teorema 4.25 Toda superficie compacta $\mathcal{H}$ (orientable o no) con un número par de componentes conexas en la frontera y característica de Euler par, admite una triangulación de Whitney (5-coafín) divergente en sumandos. 
Prueba. Supongamos primero que $\mathcal{H}$ es una superficie cerrada (sin frontera) Cualquiera que se el caso, podemos construir una triangulación como la que necesitamos con alguna de las dos construcciones siguientes (y a veces con ambas):

Caso 1. $\mathcal{H}$ no es orientable ó $\mathcal{H}$ es orientable con $\chi(\mathcal{H})=4 m+2$.

Sea $W$ cualquier triangulación de Whitney que satisfaga $\chi(W)=\frac{1}{2} \chi(\mathcal{H})+1 \mathrm{y}$ que sea orientable o no según lo sea $\mathcal{H}$. Observe que la condición $\chi(\mathcal{H})=4 m+2$ es necesaria para que $\chi(W)$ sea par y así poder asegurar que tal superficie existe en el caso orientable.

Elimine una arista cualquiera de $W$ para obtener $W_{1}$ y sea $C_{1}$ el cuadrado inducido de $W_{1}$ que se formó al eliminar la arista de $W$. Sea $W_{2}$ una copia disjunta de $W_{1}$ y $C_{2}$ el cuadrado que le corresponde a $C_{1}$. Si ahora tomamos la unión disjunta de $W_{1}, \mathbb{S}_{1}$ y $W_{2}$ e identificamos los cuadrados $C_{1}$ con $\{1, a, x, b\}$ y $C_{2}$ con $\{y, d, 4, c\}$ obtenemos una triangulación de Whitney 5 -coafín divergente en sumandos de una superficie $\mathcal{S}$ con $\chi(\mathcal{S})=2 \chi(W)+2-4=\chi(\mathcal{H})$ y $\mathcal{S}$ es orientable o no según lo sea $\mathcal{H}$. Es decir, $\mathcal{S}$ y $\mathcal{H}$ son homeomorfas.

Caso 2. $\mathcal{H}$ no es orientable con $\chi(\mathcal{H}) \leq-2$ ó $\mathcal{H}$ es orientable con $\chi(\mathcal{H})=4 m$.

Sea $W$ cualquier triangulación de Whitney que satisfaga $\chi(W)=\frac{1}{2} \chi(\mathcal{H})+2$ y que sea orientable o no según lo sea $\mathcal{H}$. Observe que la condición $\chi(\mathcal{H})=4 m$ es necesaria para que $\chi(W)$ sea par en el caso orientable y que la condición $\chi(\mathcal{H}) \leq-2$ es necesaria en el caso no orientable, pues de lo contrario $W$ tendría que ser una triangulación no orientable con $\chi(W)=2$.

Sea $\mathbb{T}_{1}$ la gráfica que obtenemos de $\mathbb{T}$, al eliminar las aristas $\{b, u\}$ y $\{c, v\}$ que están totalmente contenidas en la subgráfica $\mathbb{S}_{1}$ de $\mathbb{T}$. Observamos que $\mathbb{T}_{1}$ todavía es 5 -coafín divergente en sumandos. Sean $W_{1}, C_{1}, W_{2}$ y $C_{2}$ como en la construcción anterior. Ahora obtenemos una triangulación de Whitney 5 -coafín divergente en sumandos al tomar la unión disjunta de $W_{1}, \mathbb{T}_{1}$ y $W_{2}$ e identificar los cuadrados $C_{1}$ con $\{b, p, u, 1\}$ y $C_{2}$ con $\{c, q, v, y\}$. Con esta otra construcción, obtenemos una triangulación de una superficie $\mathcal{S}$ con $\chi(\mathcal{S})=$ $2 \chi(W)+0-4=\chi(\mathcal{H})$ tal que $\mathcal{S}$ es orientable o no según lo sea $\mathcal{H}$. De nuevo tenemos que $\mathcal{S}$ y $\mathcal{H}$ son homeomorfas.

Si ahora deseamos dar triangulaciones para $\mathcal{H}$ en el caso en que $\mathcal{H}$ tenga un número par de componentes conexas en la frontera, basta con repetir las construcciones 
anteriores tomando $W$ con la mitad de componentes conexas en la frontera que las que tiene $\mathcal{H}$.

Teorema 4.26 Toda superficie compacta no orientable con un cualquier número de componentes conexas en la frontera admite una triangulación de Whitney $k$-divergente.

Prueba. En las triangulaciones obtenidas del teorema anterior, si identificamos a todo vértice con su imagen bajo la coafinación, obtenemos de nuevo una triangulaciones de Whitney: No se forman triángulos adicionales pues la coafinación es 5-coafín (bastaría que fuera 4-coafín). Las nuevas triangulaciones de superficies así obtenidas son $k$-divergentes gracias al teorema del cubrimiento triangular (teorema 1.13). Es fácil verificar que las nuevas superficies resultantes no son orientables y que cubren todos los casos de característica impar que estaban ausentes en el teorema anterior.

En particular, hemos probado que:

Teorema 4.27 Toda superficie cerrada admite una triangulación $k$-divergente. 


\section{Capítulo 5}

\section{Cuello local grande}

El tetraedro es la única gráfica localmente $\mathcal{C}_{3}$ y claramente es $k$-nulo. El octaedro y el icosaedro son respectivamente las únicas gráficas localmente $\mathcal{C}_{4}$ y $\mathcal{C}_{5}$ y ambas son $k$-divergentes [56,61]. Luego vienen las gráficas localmente $\mathcal{C}_{6}$ que se sabe que hay un número infinito de ellas y todas son $k$-divergentes [39,40]. ¿Qué pasa con las gráficas localmente $\mathcal{C}_{t}$ para $t \geq 7$ ?

En este capítulo responderemos a esta cuestión: Toda gráfica localmente $\mathcal{C}_{t}$ con $t \geq 7$ es clan convergente $\ldots$ más aún, toda gráfica localmente $\left\{\mathcal{C}_{t}: t \geq 7\right\}$ es clan convergente ... incluso más: toda gráfica con cuello local al menos 7 es clan convergente.

La mayoría de los resultados presentados aquí fueron reportados originalmente en [45].

\section{Cuello local}

Sea $G$ una gráfica, el cuello de $G$, cuello $(G)$, se define como la longitud de un ciclo de longitud mínima de $G$. Si $G$ no tiene ciclos decimos que cuello $(G)=\infty$. Como nosotros sólo consideramos gráficas simples, cuello $(G) \geq 3$.

Si $x \in V(G)$, el cuello local de $G$ en $x$ es: $c l(G, x)=$ cuello $\left(N_{G}(x)\right)$.

El cuello local de $G$ es $c l(G)=\min \{c l(G, x): x \in V(G)\}$. 


\section{El teorema de triángulos y vértices}

El objetivo principal de esta sección es probar el siguiente teorema que será útil después para probar el teorema del cuello local grande (teorema 5.3).

Teorema 5.1 Sea $G$ una gráfica con $\mathrm{cl}(G) \geq 7$ y sea $\mathcal{F}$ una familia de al menos 3 triángulos y/o vértices de $G$ que satisface las siguientes propiedades:

1. Cada vez que tenemos dos triángulos distintos $T_{1}$ y $T_{2}$ de $\mathcal{F}$ se satisface que $\left|T_{1} \cap T_{2}\right|=2$ o bien $\left|T_{1} \cap T_{2}\right|=1$ y existe un triángulo $T_{3}$ de $G$ tal que $\left|T_{1} \cap T_{3}\right|=2$ y $\left|T_{2} \cap T_{3}\right|=2$.

2. Si $T$ y $v$ son respectivamente un triángulo y un vértice de $\mathcal{F}$, entonces $v \in T$ $o ́ N_{G}[v] \cap T \mid=2$.

3. Si u y $v$ son dos vértices de $\mathcal{F}$, entonces $u$ y $v$ son adyacentes en $G$.

Entonces existe un triángulo $T_{0}$ en $G$ tal que:

1. Para todo triángulo $T$ en $\mathcal{F}, T_{0}=T$ ó $\left|T \cap T_{0}\right|=2$.

2. Para todo vértice $v$ en $\mathcal{F}, v \in T_{0}$.

Prueba. Estudiaremos la situación en varios casos. Los casos 3 y 4 cubren todas las posibilidades, pero el caso 1 se usará para probar el caso 2 y ambos para probar el caso 3 .

Caso 1. Existen dos triángulos $T_{1}, T_{2} \in \mathcal{F}$ tal que $\left|T_{1} \cap T_{2}\right|=1$.

Sean $T_{1}=\{a, b, c\}$ y $T_{2}=\left\{a, b^{\prime}, c^{\prime}\right\}$. Por hipótesis existe un triángulo $T_{3}$ de $G$ tal que $\left|T_{1} \cap T_{3}\right|=2$ y $\left|T_{2} \cap T_{3}\right|=2$. Sin pérdida de generalidad, digamos que $T_{3}=\left\{a, b, b^{\prime}\right\}$.

Afirmamos que $T_{0}=T_{3}$ funciona:

Sea $T \neq T_{1}, T_{2}$ un triángulo en $\mathcal{F}$. Probaremos que $T=T_{0}$ ó $\left|T \cap T_{0}\right|=2$. Supongamos que $\left|T \cap T_{0}\right| \leq 1$ para llegar a una contradicción. 
Caso $1.1 a \in T$.

Si $\left|T \cap T_{1}\right|=\left|T \cap T_{2}\right|=1$ entonces, digamos que $T=\{a, u, v\}$ con $\{u, v\} \cap$ $\left\{a, b, c, b^{\prime}, c^{\prime}\right\}=\varnothing$ (recuerde que hemos supuesto que $\left|T \cap T_{0}\right| \leq 1$ ). Entonces, por hipótesis tienen que existir otros dos triángulos $T_{4}$ y $T_{5}$ tal que $\left|T \cap T_{4}\right|=$ $\left|T_{1} \cap T_{4}\right|=2=\left|T \cap T_{5}\right|=\left|T_{2} \cap T_{5}\right|$. Esto garantiza que hay (al menos) una arista que conecta a los conjuntos de vértices $\{u, v\}$ y $\{b, c\}$ y también al menos una arista entre los conjuntos $\{u, v\}$ y $\left\{b^{\prime}, c^{\prime}\right\}$. Pero entonces, el conjunto de seis vértices $\left\{u, v, b, b^{\prime}, c, c^{\prime}\right\}$ induce al menos 6 aristas (además de las dos mencionadas se tenían ya: $\{c, b\},\left\{b, b^{\prime}\right\},\left\{b^{\prime}, c^{\prime}\right\}$ y $\left.\{u, v\}\right)$ y por lo tanto tiene que contener un ciclo de longitud menor o igual a 6 . Como $\left\{u, v, b, b^{\prime}, c, c^{\prime}\right\} \subseteq N_{G}(a)$, tenemos una contradicción con la hipótesis de cuello local grande.

Supongamos ahora que $T$ intersecta en dos vértices a (exactamente) uno de los triángulos $T_{1}$ o $T_{2}$. Sin pérdida de generalidad, suponemos que $\left|T \cap T_{1}\right|=1$ y $\left|T \cap T_{2}\right|=2$. Como $\left|T \cap T_{0}\right| \leq 1$, tendríamos que $T=\left\{a, c^{\prime}, u\right\}$ con $u \notin$ $\left\{a, b, c, b^{\prime}, c^{\prime}\right\}$. Ahora, por hipótesis tendríamos que existe un triángulo $T_{5}$ tal que $\left|T \cap T_{5}\right|=\left|T_{5} \cap T_{1}\right|=2$. Esto implica que hay por lo menos una arista que conecta los conjuntos de vértices $\{c, b\}$ y $\left\{u, c^{\prime}\right\}$. Luego el conjunto de 5 vértices $\left\{u, b, c, b^{\prime}, c^{\prime}\right\}$ induciría por lo menos 5 aristas (la recién mencionada además de $\{c, b\},\left\{b, b^{\prime}\right\},\left\{b^{\prime}, c^{\prime}\right\}$ y $\left.\left\{c^{\prime}, u\right\}\right)$ y entonces la vecindad de $a$ contiene un ciclo de longitud menor o igual que 5.

Finalmente, si $\left|T \cap T_{1}\right|=\left|T \cap T_{2}\right|=2$, como $\left|T \cap T_{0}\right| \leq 1$, tendríamos que $T=\left\{a, c, c^{\prime}\right\}$ y entonces el conjunto $\left\{c, b, b^{\prime}, c^{\prime}\right\}$ induciría un ciclo de longitud 4 en la vecindad de $a$.

Caso $1.2 a \notin T$.

Entonces, como $T$ tiene que intersectar en por lo menos un vértice tanto a $T_{1}$ como a $T_{2}$ y por lo tanto hay por lo menos una arista de $T_{1} \cap T$ a $T_{2} \cap T$. Como $\left|T \cap T_{0}\right| \leq 1$, esa arista no puede ser $\left\{b, b^{\prime}\right\}$ así que el conjunto de 4 vértices $\left\{c, b, b^{\prime}, c^{\prime}\right\}$ induce al menos 4 aristas y a contiene un ciclo de longitud a lo más 4 en su vecindad.

Hemos probado pues que todo triángulo $T$ en $\mathcal{F}$ satisface $T=T_{0}$ ó $\left|T \cap T_{0}\right|=2$. Veamos que ocurre con los vértices: 
Sea $v$ un vértice en $\mathcal{F}$. Si $v \in T_{0}$ no hay nada que probar. Supongamos que $v \in\left(T_{1} \cup T_{2}\right)-T_{0}$. Sin pérdida de generalidad, suponemos que $v \in T_{1}-T_{0}$, es decir $v=c$. Entonces, $v$ tiene que ser adyacente a dos vértices de $T_{2}$ (uno de los cuales, por cierto, es $a$ ) y el conjunto de 4 vértices $\left\{c, b, b^{\prime}, c^{\prime}\right\}$ induce al menos 4 aristas y por lo tanto, a contiene un ciclo de longitud a lo más 4 en su vecindad.

Finalmente, supongamos que $v \notin T_{1} \cup T_{2} \cup T_{0}$. Entonces, $v$ tiene que ser adyacente a dos vértices de $T_{1}$ y a dos vértices de $T_{2}$. Si $v$ es adyacente a $a$, $a$ tiene un ciclo de longitud a lo más 5 en su vecindad. Por el contrario, si $v$ no es adyacente a $a, v$ tiene que ser adyacente a $c, b, b^{\prime}$ y $c^{\prime}$. En este caso, $b$ tiene un ciclo de longitud 4 en su vecindad.

Caso 2. Existen un triángulo $T_{1}$ y un vértice $v$ en $\mathcal{F}$ tal que $\left|N_{G}[v] \cap T_{1}\right|=2$.

Si las condiciones del caso 1 se dan, no hay nada más que probar. Así que suponemos que $\left|T \cap T^{\prime}\right|=2$ para cualesquiera dos (si es que hay dos) triángulos $T, T^{\prime}$ en $\mathcal{F}$. Sean $T_{1}=\{a, b, c\}$ y $v \notin\{a, b, c\}$. Sin pérdida de generalidad, $N_{G}[v] \cap T_{1}=\{a, b\}$. Afirmamos que el triángulo $T_{0}=\{a, b, v\}$ nos sirve:

Supondremos que $T$ es un triángulo en $\mathcal{F}$ con $\left|T \cap T_{0}\right| \leq 1$ para llegar a una contradicción. Entonces, como $\left|T \cap T_{1}\right|=2$ pero $\left|T \cap T_{0}\right| \leq 1$, sin pérdida de generalidad podemos suponer que $T=\{a, c, x\}$ con $x \notin\{a, b, c\}$. Entonces hay dos posibilidades: $v \in T$ ó $v \notin T$ y $\left|N_{G}[v] \cap T\right|=2$. En el primer caso, la vecindad de $a$ contiene un ciclo de longitud 3 (contradiciendo la hipótesis de cuello local grande). En el segundo caso, el conjunto de 4 vértices $\{v, b, c, x\}$ induce al menos 4 aristas y $a$ tiene un ciclo de longitud a lo más 4 en su vecindad.

Si $v_{1}$ es otro vértice de $\mathcal{F}$, tiene que ser adyacente a $v$ y entonces, podemos suponer que $v_{1} \notin\{v, a, b, c\}$, pues si $v_{1} \in\{a, b, v\}=T_{0}$ no hay nada que probar y si $v_{1}=c, v$ tendría un ciclo de longitud 3 en su vecindad.

Entonces $v_{1}$ tiene que ser adyacente a $v$ y a dos vértices de $T_{1}$. Sin pérdida de generalidad, $v_{1}$ es adyacente a $a$ y entonces a contiene un ciclo de longitud 3 ó 4 en su vecindad.

Caso 3. Hay un triángulo en $\mathcal{F}$.

Si las condiciones del caso 1 ó 2 se dan no hay nada más que probar. 
Suponemos entonces que para cualesquiera dos triángulos distintos $T_{1}$ y $T_{2}$ de $\mathcal{F}$ se cumple que $\left|T_{1} \cap T_{2}\right|=2$ y que para cualquier vértice $v \in \mathcal{F}$ y cualquier triángulo $T_{1} \in \mathcal{F}$, tenemos que $v \in T_{1}$. Pero entonces cualquier triángulo $T_{0}$ de $\mathcal{F}$ satisface las conclusiones del teorema.

Caso 4. $\mathcal{F}$ no tiene triángulos.

Si $\mathcal{F}$ consta exclusivamente de vértices, entonces todos ellos deben ser adyacentes. Por hipótesis, deben ser al menos 3. No pueden ser más de 3 porque inducirían un tetraedro y cualquiera de estos vértices contendría un ciclo de longitud 3 en su vecindad. Así que son exactamente tres vértices adyacentes $\mathcal{F}=\{u, v, w\}$. El triángulo inducido $T_{0}=\{u, v, w\}$, satisface las conclusiones del teorema.

\section{El teorema del cuello local grande}

Para poder probar el teorema del cuello local grande, necesitamos conocer los clanes de clanes de las gráficas que nos ocupan $(\operatorname{cl}(G) \geq 7)$. Suponiendo solamente que $c l(G) \geq 5$, podremos describir detalladamente los clanes de clanes de $G$ :

Teorema 5.2 Si $G$ es una gráfica con $c l(G) \geq 5$, entonces:

1. Toda estrella $P \in k^{2}(G)$ de $G$ es de la forma $P=x^{*}$ para alguna $x \in G$.

2. Toda corbata $Q \in k^{2}(G)$ de $G$ es de la forma $Q_{T}=\left\{T^{\prime} \in k(G):\left|T^{\prime} \cap T\right| \geq 2\right\}$, para algún triángulo $T$ de $G$.

3. Dos estrellas $x^{*}$ y $y^{*}$ son adyacentes en $k^{2}(G)$ ssi $x$ y $y$ son adyacentes en $G$.

4. Dos corbatas $Q_{T_{1}}, Q_{T_{2}}$ son adyacentes en $k^{2}(G)$ ssi $\left|T_{1} \cap T_{2}\right|=2$ ó $\left|T_{1} \cap T_{2}\right|=1$ $y$ existe un triángulo $T_{3}$ tal que $\left|T_{1} \cap T_{3}\right|=2=\left|T_{2} \cap T_{3}\right|$.

5. Una estrella $x^{*}$ y una corbata $Q_{T}$ son adyacentes ssi $x \in T$ ó $\left|N_{G}[x] \cap T\right|=2$.

Prueba. Observamos que todo clan de $G$ es un triángulo o una arista que no está contenida en un triángulo (bastaría incluso que $c l(G) \geq 4$ ). Los clanes de $k(G)$ pueden ser estrellas o corbatas. 
Si $Q \in k^{2}(G)$ es una estrella de $G$, entonces $Q=x^{*}$ para alguna $x \in G$ (ver pág. 9). Claramente, dos estrellas $x^{*}, y^{*}$ de $G$ son adyacentes en $k^{2}(G)$ si y solamente si sus centros $x$ y $y$ son adyacentes en $G$.

Sea $Q \in k^{2}(G)$ una corbata de $G$. En primer lugar observamos que $Q$ no puede contener a un clan que sea una arista: Si $\{a, b\} \in Q$, debe haber un clan $q_{1} \in Q$ tal que $q_{1} \cap\{a, b\}=\{a\}$ y otro clan $q_{2} \in Q$ tal que $q_{2} \cap\{a, b\}=\{b\}$, pero ahora $q_{1}$ y $q_{2}$ deben intersectarse en algún otro vértice, digamos $x \in q_{1} \cap q_{2}$. Ahora, resulta que $\{a, b, x\}$ es una completa, contradiciendo el hecho de que $\{a, b\}$ es un clan. Concluimos que $Q$ consta exclusivamente de triángulos.

Si todos los triángulos de $Q$ se intersectaran (dos a dos) en una arista, tendríamos dos posibilidades: o bien hay una arista que está contenida en todos los triángulos de $Q$ (contradiciendo el que $Q$ es una corbata) o bien $Q$ contiene tres triángulos que inducen un tetraedro (contradiciendo la hipótesis de cuello local grande). Así que en $Q$ debe haber al menos dos triángulos $T_{1}$ y $T_{2}$ que se intersectan exactamente en un vértice, digamos $T_{1}=\{a, b, x\}$ y $T_{2}=\{u, v, x\}$.

Como $Q$ es una corbata, debe haber otro clan $T_{3}$ en $Q$ tal que $x \notin T_{3}$ y sin embargo, $T_{3}$ debe intersectar no trivialmente a $T_{1}$ y $T_{2}$. Sin pérdida de generalidad, sea $T_{3}=$ $\{a, u, z\}$.

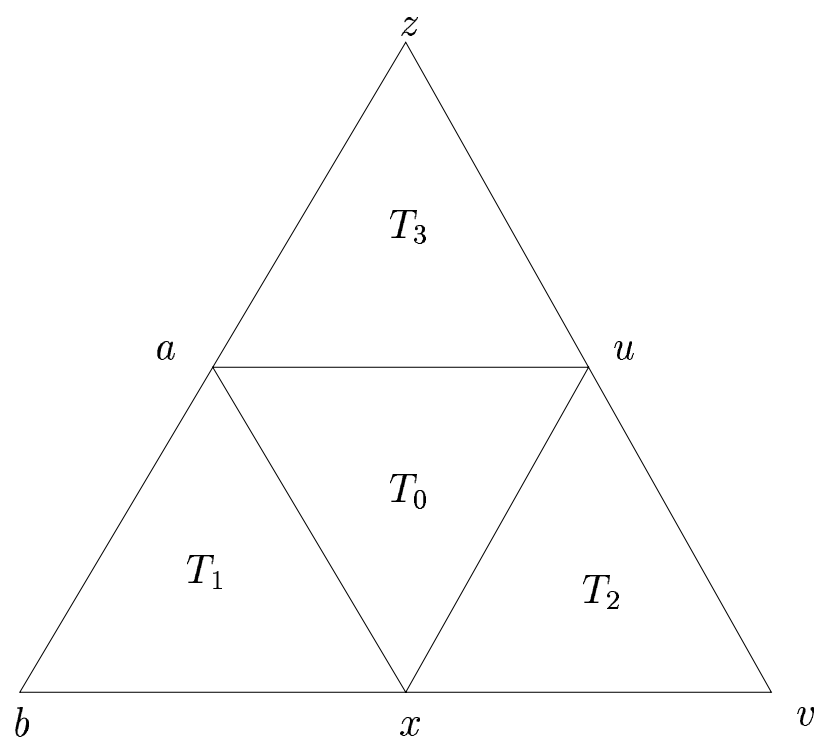

Figura 5.1: Una corbata de $G$ 
Observamos que $z \notin\{a, b, x, u, v\}$, pues de lo contrario, tendríamos $x \in T_{3}, c l(G, u)=$ 3 ó $\operatorname{cl}(G, a)=3$.

Sea $T_{0}=\{a, u, x\}$. Observamos que $T_{0} \in Q$, pues de lo contrario, tendría que haber un triángulo $T_{4} \in Q$ tal que $T_{0} \cap T_{4}=\varnothing$, pero entonces, necesariamente tendríamos $T_{4}=\{b, z, v\}$ y $\operatorname{cl}(G, x) \leq 4$. Contradicción.

Hasta ahora tenemos que $\left\{T_{0}, T_{1}, T_{2}, T_{3}\right\} \subseteq Q$

Sea ahora $T$ cualquier otro triángulo de $Q$. Si $T$ intersectara a $T_{0}$ en un único vértice, digamos $x$, tendríamos que $T \cap T_{3}=\{z\}$ y ello implica que $c l(G, u)=3$. De modo que $T$ intersecta a $T_{0}$ en exactamente dos vértices.

Hemos probado que $Q$ tiene un triángulo especial $T_{0}$ y que cualquier otro triángulo de $Q$ tiene que intersectar a $T_{0}$ en exactamente 2 vértices. También, hemos visto que para cada arista de $T_{0}$ hay al menos un triángulo $T \in Q$ que se intersecta con $T_{0}$ exactamente en esa arista.

Recíprocamente, si $T$ es un triángulo de $G$, la colección $Q_{T}=\left\{T^{\prime} \in k(G):\left|T \cap T^{\prime}\right| \geq\right.$ 2 \} es una corbata de $G$ siempre y cuando para cada arista de $T$ exista un triángulo en $G$ que intersecte a $T$ en exactamente esa arista.

Las caracterizaciones de adyacencias enunciadas son ahora obvias.

Decimos que $x$ es el centro de $x^{*}$ (aunque el centro pueda no ser único) y que $T$ es el centro de $Q_{T}$.

Si $G$ es una triangulación de Whitney de una superficie $(\operatorname{cl}(G) \geq 5)$, toda corbata de $G$ es exactamente como en la figura 5.1. En general, una corbata puede tener más de 4 triángulos como se muestra en la figura 5.2. Cuando $\operatorname{cl}(G) \nsupseteq 5$, las corbatas pueden ser incluso más extrañas y pueden no tener nada que podamos llamar centro. Si está interesado en corbatas exóticas, puede estudiar las que aparecen en el octaedro.

Teorema 5.3 (Teorema del cuello local grande) Si $G$ es una gráfica con $\operatorname{cl}(G) \geq 7$, entonces $k(G)$ es clan-Helly y por lo tanto $G$ es $k$-convergente.

Prueba. Sea $\mathcal{F}$ una familia de clanes de $k(G)$ (vértices en $k^{2}(G)$ ) que se intersectan 2 a 2 . Tenemos que probar que toda la familia se intersecta de manera no trivial. 


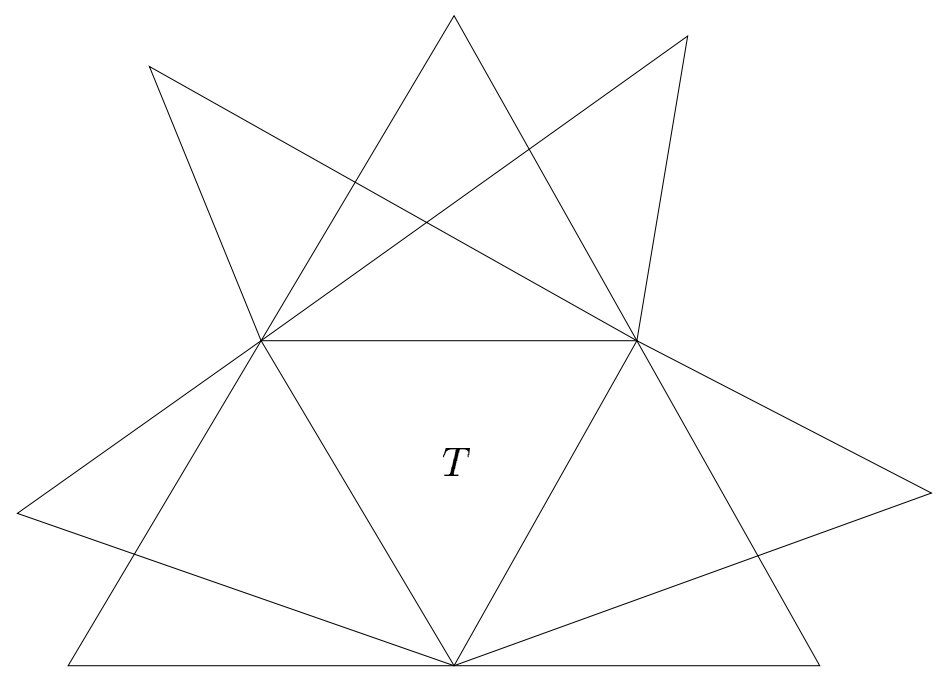

Figura 5.2: Otra corbata de $G$ con centro en $T$

Por el teorema anterior, $\mathcal{F}=\left\{Q_{T_{1}}, \ldots, Q_{T_{r}}, x_{1}^{*}, \ldots, x_{s}^{*}\right\}$ para algunos triángulos $T_{i}$ y algunos vértices $x_{j}$ de $G$. También por el teorema anterior, la familia $\mathcal{F}^{\prime}=$ $\left\{T_{1}, \ldots, T_{r}, x_{1}, \ldots, x_{s}\right\}$ satisface las condiciones 1-3 del teorema 5.1.

Si $\left|\mathcal{F}^{\prime}\right| \leq 2$ no hay nada que probar, de lo contrario el teorema 5.1 nos dice que hay un triángulo $T_{0}$ tal que $T_{0} \in x^{*}$ y $T_{0} \in Q_{T}$ para cualquier estrella $x^{*}$ ó corbata $Q_{T}$ que pertenezcan a $\mathcal{F}$. De modo que $\mathcal{F}$ tiene una intersección común. Por el teorema de Escalante (teorema 1.7), $G$ es clan-convergente.

\section{Superficies $k$-convergentes}

De inmediato se siguen varios interesantes corolarios al teorema del cuello local grande:

Teorema 5.4 Toda gráfica localmente $\mathcal{C}_{t}$ con $t \geq 7$ es $k$-convergente.

Teorema 5.5 Toda gráfica localmente $\left\{\mathcal{C}_{t}: t \geq 7\right\}$ es $k$-convergente.

Teorema 5.6 Toda triangulación de Whitney de una superficie compacta (con o sin frontera) con grado al menos 7 en todos los vértices interiores es $k$-convergente. 
Por supuesto, la aplicabilidad de los teoremas previos depende de la existencia y de la cantidad de gráficas localmente cíclicas. De hecho se prueba en [45] que para cada $t \geq 7$ existen infinitas superficies no homeomorfas que admiten una triangulación de Whitney localmente $C_{t}$.

A continuación veremos una construcción explícita de suficientes gráficas localmente $\mathcal{C}_{7}$ como para triangular infinitas superficies no homeomorfas. Este material fue presentado originalmente en [42].

Empezaremos por construir una familia de gráficas localmente $\mathcal{C}_{6}$ (triangulaciones del toro) y a partir de ellas construiremos todas las gráficas localmente $\mathcal{C}_{7}$ que necesitamos.

Considere la gráfica infinita $T$ con vértices en el grupo abeliano libre $V(T)=\mathbb{Z} \oplus \mathbb{Z} \mathrm{y}$ aristas dadas por $E(T)=\{\{u, v\}: u, v \in \mathbb{Z} \oplus \mathbb{Z}$ y $u-v \in\{ \pm(1,0), \pm(0,1), \pm(1,-1)\}\}$. Esta gráfica es localmente $\mathcal{C}_{6}$ y es la cubierta universal triangular de todas las gráficas localmente $\mathcal{C}_{6}$ finitas tal y como se prueba en [40]. Observamos que cualquier vector $v \in \mathbb{Z} \oplus \mathbb{Z}$ (vértice de $T$ ) tiene asociada una traslación $\tau_{v}$ en $\mathbb{Z} \oplus \mathbb{Z}$ dada por $\tau_{v}(x)=x+v$.

Tomemos ahora un entero fijo $r \geq 2$. Sean $u=(4,1), v=(2 r, 4 r), \alpha=\tau_{u}, \beta=\tau_{v} \mathrm{y}$ sea $\Gamma_{r}=\langle\alpha, \beta\rangle$ : el grupo generado por $\alpha \mathrm{y} \beta$. Si ahora identificamos dos vértices $x \mathrm{y}$ $y$ en $T$ cada vez que exista una $\gamma \in \Gamma_{r}$ con $\gamma(x)=y$, obtenemos una gráfica finita (la gráfica cociente) a la que llamamos $T_{r}=T / \Gamma_{r}$. Equivalentemente, la construcción puede hacerse sobre el paralelogramo fundamental (ver figura 5.3 para el caso $r=2$ ), identificando lados opuestos. Observamos que esta gráfica es una triangulación del toro, es localmente $\mathcal{C}_{6}$ y tiene $14 r$ vértices.

Sean ahora $\omega_{1}=(2,1), \ldots, \omega_{i}=(i+1,2 i-1), \ldots, \omega_{2 r}=(2 r+1,4 r-1)$. Observamos que el conjunto de las vecindades cerradas de estos vértices en $T_{r},\left\{N\left[\omega_{1}\right], \ldots, N\left[\omega_{2 r}\right]\right\}$, es una partición del conjunto de vértices de $T_{r}$. Por lo tanto, si removemos los vértices $\omega_{1}, \ldots, \omega_{2 r}$ de $T_{r}$ obtenemos una gráfica $T_{r}^{\prime}$ localmente $\mathcal{P}_{5}: T_{r}^{\prime}=T_{r}-\left\{\omega_{1}, \ldots, \omega_{2 r}\right\}$ con $12 r$ vértices. $T_{r}^{\prime}$ es una triangulación de Whitney de un toro menos $2 r$ discos. Para $i=1, \ldots, 2 r$, sea $D_{i}=N\left(\omega_{i}\right)$ la $i$-ésima componente conexa de la frontera de $T_{r}^{\prime}$. Cada $D_{i}$ induce un ciclo de longitud 6 en $T_{r}^{\prime}$.

Consideramos ahora $r$ copias disjuntas de la triangulación del cilindro de la figura 5.4. Para $i=1, \ldots, r$, sean $B_{i}$ y $B_{r+i}$ las componentes conexas de la frontera de la 


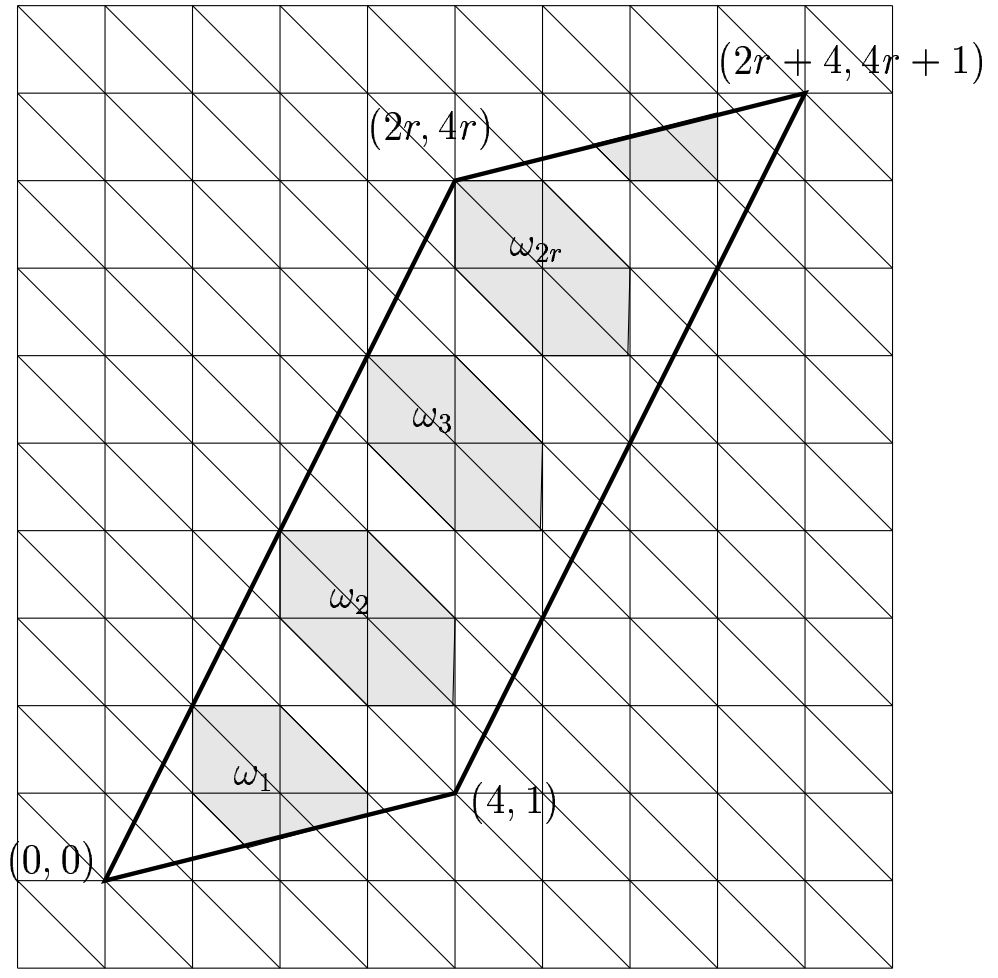

Figura 5.3: Una región fundamental en $\mathbb{Z} \oplus \mathbb{Z}$ que produce a la gráfica $T_{r}$

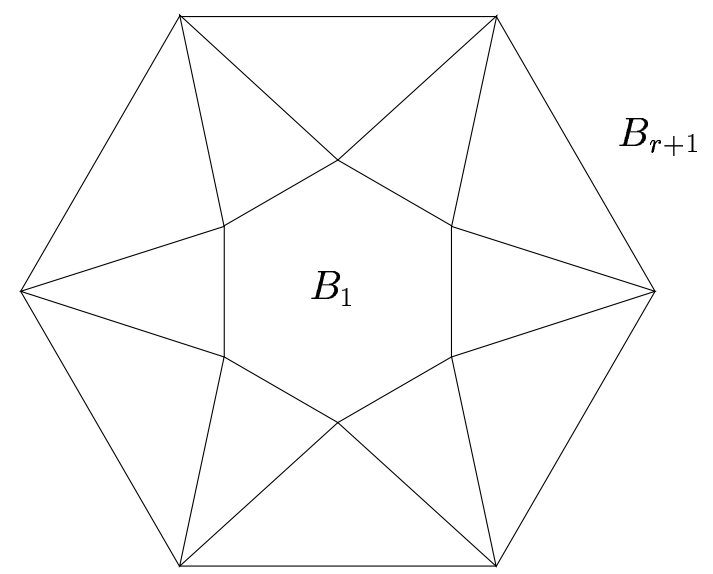

Figura 5.4: Una triangulación del cilindro localmente $\mathcal{P}_{4}$ 
$i$-ésima copia del cilindro. Ahora, identificamos los vértices de $B_{j}$ con los de $D_{j}$ para $j=1, \ldots, 2 r$ respetando el orden cíclico. A la gráfica resultante le llamamos $T^{(r)}$.

Para asegurarnos que $T^{(r)}$ es una triangulación de Whitney, hay que tener el cuidado de hacer las identificaciones de modo que no se conviertan en vecinos dos vértices de $T_{r}^{\prime}$ que estén a distancia 2 en $T_{r}^{\prime}$. En el caso $r=2$ hay una única manera de hacer las identificaciones sujetándose a esta restricción. La gráfica resultante es una triangulación del toro triple (género $=3$ ). En los demás casos $(r>2)$ hay suficiente libertad como para poder hacer las identificaciones de la manera que se prefiera y en particular, la gráfica $T^{(r)}$ puede ser una triangulación del $(r+1)$-toro o de la superficie no orientable que tiene la misma característica de Euler que el $(r+1)-$ toro.

Todas las triangulaciones así obtenidas son localmente $\mathcal{C}_{7}$ y por el teorema del cuello grande, son $k$-convergentes.

También se puede construir fácilmente una triangulación clan convergente del toro doble: En $T_{2}$ (figura 5.3) elimine la arista que une al vértice $(3,3)$ con el $(4,3)$ y llamemos $T_{2}^{\prime}$ a esta gráfica. Sea $C_{1}$ el cuadrado inducido por $\{(3,3),(4,2),(4,3),(3,4)\}$ en $T_{2}^{\prime}$. Sea $T_{2}^{\prime \prime}$ una copia disjunta de $T_{2}^{\prime}$ y sea $C_{2}$ el correspondiente cuadrado de $T_{2}^{\prime \prime}$. Tome la unión disjunta de $T_{2}^{\prime}$ y $T_{2}^{\prime \prime}$ y luego identifique los cuadrados $C_{1}$ y $C_{2}$ para obtener una triangulación de Whitney del toro doble. Esta gráfica tiene cuello local 6 y nuestros teoremas no valen para ella, pero una verificación directa (usando Gap [19]) nos muestra que es clan convergente.

Hemos probado así que toda superficie cerrada orientable con género al menos 2 y toda superficie cerrada (orientable o no) con característica de Euler par y menor o igual que -6 admite una triangulación (de Whitney) $k$-convergente.

De hecho, usando el teorema del cubrimiento triangular basta con dar un ejemplo de una triangulación de Whitney $k$-convergente para la superficie que resulta de tomar la suma conexa de un toro y un plano proyectivo, para asegurar la existencia de triangulaciones de Whitney $k$-convergentes para casi todas las superficies. Justamente esto es lo que se hizo en [42]:

Teorema 5.7 [42] Casi todas las superficies cerradas admiten una triangulación de Whitney $k$-convergente. Las posibles excepciones son: La esfera, el plano proyectivo, el toro y la botella de Klein. 
Rápidamente emerge el siguiente:

Problema 12 Determinar si existen triangulaciones de Whitney $\left(\neq K_{4}\right)$ clan convergentes para la esfera, el plano proyectivo, el toro y la botella de Klein.

Para la esfera, se tiene mucha evidencia experimental que apunta a que la respuesta debería ser negativa:

Conjetura 13 (Conjetura de la esfera [43,45]) Con excepción del tetraedro $K_{4}$, toda triangulación de Whitney de la esfera es $k$-divergente.

Si la conjetura de la esfera es cierta, usando el teorema del cubrimiento triangular se puede probar que lo mismo sucede con el plano proyectivo.

Una interesante conjetura relacionada es:

Conjetura 14 (Conjetura del disco [43,45]) Toda triangulación de Whitney del disco es $k$-nula.

Gracias al teorema del cuello local grande, conocemos el $k$-carácter de las gráficas localmente $\left\{\mathcal{C}_{t}: t \geq 7\right\}$ y por otro lado, también conocemos el $k$-carácter de las gráficas localmente $\mathcal{C}_{t}$ para $t \leq 6$. Es natural preguntarse qué pasa con las otras gráficas localmente cíclicas de grado mixto, como por ejemplo las gráficas localmente $\left\{\mathcal{C}_{6}, \mathcal{C}_{7}\right\}$ o las localmente $\left\{\mathcal{C}_{4}, \mathcal{C}_{5}, \mathcal{C}_{6}\right\}$, etc. Más aún, es natural preguntarse:

Problema 15 Determinar el k-carácter de todas las gráficas localmente cíclicas.

En particular: hay un número finito de gráficas localmente $\left\{\mathcal{C}_{4}, \mathcal{C}_{5}\right\}$. Estas gráficas se muestran en la figura 5.5. Ya sabemos que el octaedro y el icosaedro son $k$-divergentes, por otro lado, se puede probar que las gráficas (b), (d) y (e) son expansivas (i.e. 2-coafines y divergentes en sumandos), de modo que el siguiente problema es equivalente al problema 7 (página 45).

Problema 16 Determinar el $k$-carácter de todas las gráficas localmente $\left\{\mathcal{C}_{4}, \mathcal{C}_{5}\right\}$.

La siguiente sección presenta una respuesta parcial a la conjetura del disco. 


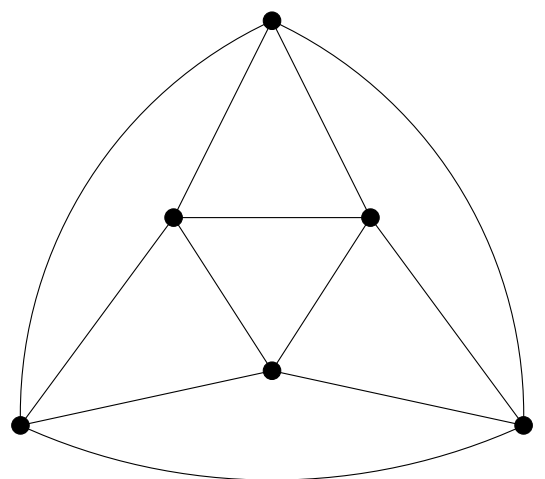

(a) Octaedro

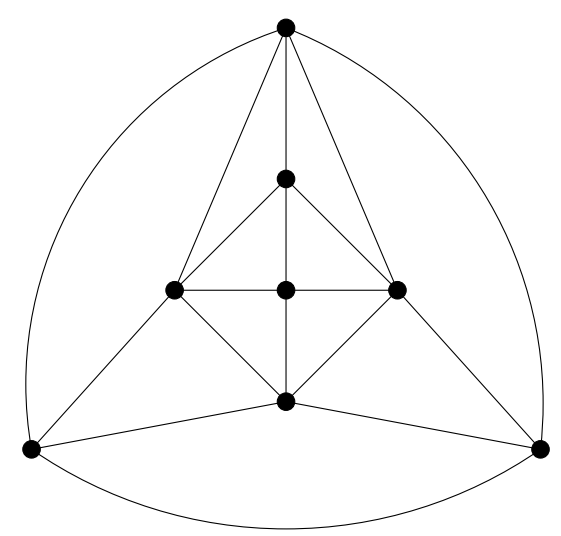

(c) La gráfica de la figura 3.1

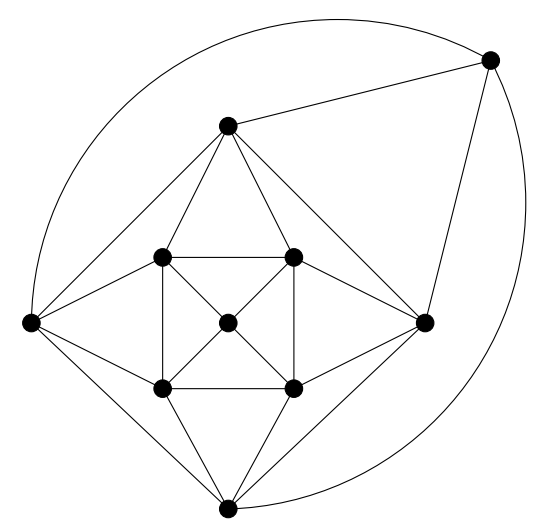

(e) ... y otra más.

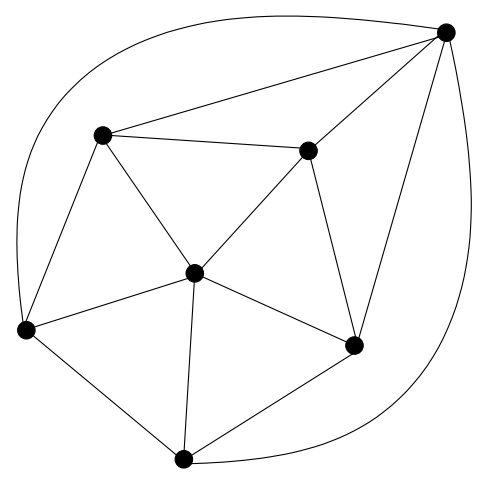

(b) Doble cono sobre $\mathcal{C}_{5}$

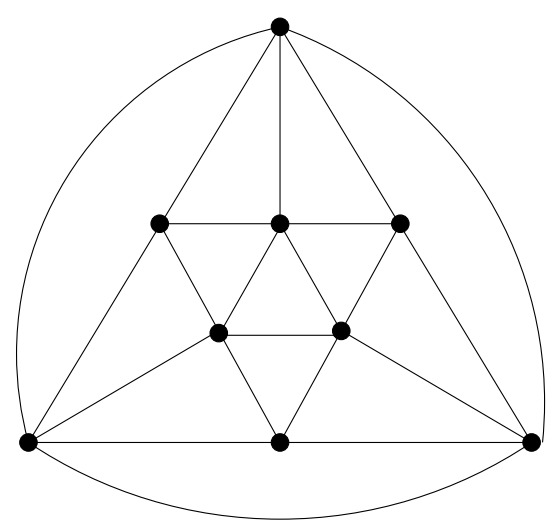

(d) Otra gráfica localmente $\left\{\mathcal{C}_{4}, \mathcal{C}_{5}\right\} \ldots$

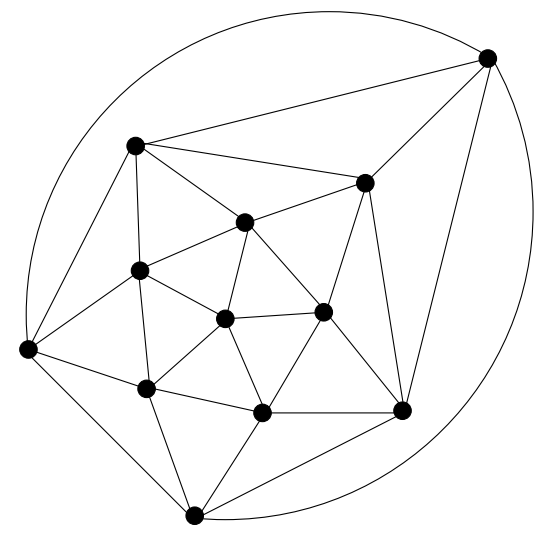

(f) Icosaedro

Figura 5.5: Todas las gráficas localmente $\left\{\mathcal{C}_{4}, \mathcal{C}_{5}\right\}$ 


\section{Triangulaciones del disco}

Teorema 5.8 Sea A una triangulación de Whitney del disco. Sea i el número de vértices interiores de $A$ y sea $f$ el número de vértices exteriores (en la frontera). Sean $\bar{d}_{i}$ y $\bar{d}_{f}$ el promedio de los grados de los vértices interiores y exteriores respectivamente. Entonces $\bar{d}_{f}=\frac{i}{f}\left(6-\bar{d}_{i}\right)-\frac{6}{f}+4$.

Prueba. Contando la cara externa tenemos, por Euler: $\chi=V-A+C=2$. Con $V=i+f, A=\frac{1}{2}\left(i \cdot \bar{d}_{i}+f \cdot \bar{d}_{f}\right)$ y si $T$ es el número de triángulos, tenemos: $i \cdot \bar{d}_{i}+f \cdot \bar{d}_{f}=3 T+f$ (es decir, la suma de grados es igual al número de caras contadas tantas veces como vértices contengan), luego $C=T+1=\frac{1}{3}\left(i \cdot \bar{d}_{i}+f \cdot \bar{d}_{f}-f\right)+1$ y entonces:

$$
\begin{aligned}
2 & =i+f-\frac{i \cdot \bar{d}_{i}+f \cdot \bar{d}_{f}}{2}+\frac{i \cdot \bar{d}_{i}+f \cdot \bar{d}_{f}-f}{3}+1 \\
& =i+\frac{2}{3} f-\frac{1}{6}\left(i \cdot \bar{d}_{i}+f \cdot \bar{d}_{f}\right)+1
\end{aligned}
$$

Luego: $6=6 i+4 f-i \cdot \bar{d}_{i}-f \cdot \bar{d}_{f}$ y despejando obtenemos: $\bar{d}_{f}=\frac{i}{f}\left(6-\bar{d}_{i}\right)-\frac{6}{f}+4$.

Teorema 5.9 Sea A una triangulación de Whitney del disco con $\bar{d}_{i} \geq 6$. Entonces, A tiene al menos dos vértices en la frontera de grado a lo más 3.

Prueba. Si todos menos uno de los vértices de la frontera tuvieran grado por lo menos 4 y $\bar{d}_{i} \geq 6$ entonces tendríamos:

$$
\bar{d}_{f}=\frac{i}{f}\left(6-\bar{d}_{i}\right)-\frac{6}{f}+4 \leq 4-\frac{6}{f}<4-\frac{2}{f}=\frac{4(f-1)+2}{f} \leq \bar{d}_{f}
$$

que es una contradicción.

Teorema 5.10 Sea A una triangulación de Whitney del disco tal que todo vértice interior tiene grado $\geq 6$. Entonces, A se retrae fuertemente a cualquiera de sus vértices. 
Prueba. Sea $u \in V(A)$ el vértice al que queremos retraer fuertemente, gracias al teorema anterior existe un vértice en la frontera $v \in V(A), v \neq u$ con grado a lo más 3 , pero entonces la vecindad de $v$ en $A$ induce una trayectoria de a lo más 3 vértices y $v$ se puede retraer fuertemente a alguno de sus vecinos. Si la gráfica resultante $A-\{v\}$ es una triangulación del disco, terminamos por hipótesis de inducción, en caso contrario, $A-\{v\}$ es o bien una arista o bien el resultado de pegar dos subgráficas por un punto, digamos $B$ y $C$ y el punto de pegado es el vértice al que se retrae fuertemente $v$, digamos $w$. En el caso en que $A-\{v\}$ es una sola arista, claramente, $A-\{v\}$ se retrae fuertemente a cualquiera de sus dos vértices y por lo tanto $A$ se retrae fuertemente a $u$. En caso contrario $B$ y $C$ pueden ser un par de aristas o una arista y una triangulación del disco o dos triangulaciones del disco. Sin pérdida de generalidad podemos suponer que $u \in B$, y entonces, como $C$ se retrae fuertemente a $w$ (por hipótesis de inducción o porque toda arista se retrae fuertemente a cualquiera de sus vértices), tenemos que $A-\{v\}$ se retrae fuertemente a $B$. Finalmente, $B$ se retrae fuertemente a $u$ ya sea por hipótesis de inducción o porque $B$ es una arista.

Teorema 5.11 Sea A una triangulación de Whitney del disco tal que todo vértice interior tiene grado $\geq 6$. Entonces $A$ es $k-n u l a$.

Prueba. Por el teorema anterior, $A$ se retrae fuertemente a un punto y por el teorema $1.12 A$ es $k$-nula. 


\section{Conclusiones}

En este trabajo, hemos hecho aportaciones significativas al estudio de las gráficas iteradas de clanes.

Expusimos nuevas y poderosas técnicas para decidir la clan divergencia de una amplia familia de gráficas (capítulo 4) y también presentamos nuevas técnicas para el estudio de la clan convergencia (capítulo 5).

Hemos incrementado el conjunto de ejemplos de gráficas clan divergentes primitivas conocidas (capítulo 3) y mostramos que el $k$-carácter de algunas familias de gráficas (relojes y gráficas regulares localmente cíclicas) puede ser determinado en tiempo polinomial (capítulos 3, 4, 5).

Ampliamos el conocimiento en la teoría de cimientos de gráficas iteradas de clanes probando varios teoremas de propósito general y en particular dimos una nueva prueba al teorema del diámetro de Bornstein y Szwarcfiter (capítulo 2).

Además hemos planteado diversos problemas abiertos que podrán servir como lineamientos para investigaciones futuras. 


\section{Problemas abiertos}

Problema 1 ¿Es cierto que $\operatorname{diam}\left(k^{n}(J(m, r))\right)=r+n$ si $2 \leq r$ y $r+n \leq\left\lfloor\frac{m}{2}\right\rfloor$ ? (Página 27)

Problema 2 ¿Es cierto que $\operatorname{diam}\left(k^{n}\left(J\left(\aleph_{0}, r\right)\right)\right)=r+n$ para toda $r \geq 2$ y $n \geq 0$ ? (Página 28)

Problema 3 ¿Existe alguna gráfica $G$ (no necesariamente finita) de diámetro finito tal que $\operatorname{diam}\left(k^{n}(G)\right)=\operatorname{diam}(G)+n$ para toda $n$ ? (Página 28)

Problema 4 (Problema del diámetro)

¿Existe alguna gráfica finita $G$ tal que $\operatorname{diam}\left(k^{n}(G)\right)=\operatorname{diam}(G)+n$ para toda $n$ ? (Página 28)

Problema 5 (Problema de la computabilidad del $k$-carácter [43])

¿Es computable el $k$-carácter? (Página 45)

Problema 6 (Problema de la computabilidad de la $k$-nulidad [43])

¿Es computable la k-nulidad? (Página 45)

Problema 7 Determinar el k-carácter de la gráfica de la figura 3.1. (Página 45)

Problema 8 (Frías y Neumann-Lara [17])

¿Es cierto que la suma punteada de dos gráficas $k$-nulas es $k$-nula? (Página 49)

Problema 9 ¿Cuál es la mínima función de crecimiento posible para una gráfica clan divergente? (Página 49) 
Problema $10 i \mathbb{A} \otimes \mathbb{B}$ es divergente en sumandos siempre que $\mathbb{A} y \mathbb{B}$ lo son? (Página 62)

Problema 11 ¿A es divergente en sumandos ssi $\lim _{n \rightarrow \infty} \operatorname{rango}\left(k^{n}(\mathbb{A})\right)=\infty$ ? (Página 62, equivalente al problema 10)

Problema 12 Determinar si existen triangulaciones de Whitney $\left(\neq K_{4}\right)$ clan convergentes para la esfera, el plano proyectivo, el toro y la botella de Klein.

(Página 82)

Conjetura 13 (Conjetura de la esfera [43,45]) Con excepción del tetraedro $K_{4}$, toda triangulación de Whitney de la esfera es k-divergente. (Página 82)

Conjetura 14 (Conjetura del disco [43,45]) Toda triangulación de Whitney del disco es k-nula. (Página 82)

Problema 15 Determinar el $k$-carácter de todas las gráficas localmente cíclicas. (Página 82)

Problema 16 Determinar el $k$-carácter de todas las gráficas localmente $\left\{\mathcal{C}_{4}, \mathcal{C}_{5}\right\}$. (Página 82, equivalente al problema 7) 


\section{Bibliografía}

[1] B.D. Acharya. Some queries on the periodicity and convergence of a graph. In Professor P. L. Bhatnagar commemoration volume, pages 185-205. Nat. Acad. Sci., Allahabad, 1979.

[2] L. Alcón, M. Gutierrez. Clique planar graphs (extended abstract). In Electronic Notes on Discrete Mathematics, volume 7, pages 6-9. Proceedings of the Brazilian Symposium on Graphs, Algorithms and Combinatorics (Fortaleza, Ceará, Brazil. March 2001), 2001.

[3] R. Balakrishnan, P. Paulraja. Self-clique graphs and diameters of iterated clique graphs. Utilitas Math. 29 (1986) 263-268.

[4] H.J. Bandelt, E. Prisner. Clique graphs and Helly graphs. J. Combin. Theory Ser. B 51 (1991) 34-45.

[5] A. Bondy, G. Durán, M.C. Lin, J.L. Szwarcfiter. A sufficient condition for selfclique graphs. In Electronic Notes on Discrete Mathematics, volume 7, pages 19-23. Proceedings of the Brazilian Symposium on Graphs, Algorithms and Combinatorics (Fortaleza, Ceará, Brazil. March 2001), 2001.

[6] J.A. Bondy, U.S.R. Murty. Graph theory with applications. American Elsevier Publishing Co., Inc., New York, 1976.

[7] C.F. Bornstein, J.L. Szwarcfiter. Clique graphs of chordal and path graphs. SIAM J. Discrete Math. 7 (1994) 331-336.

[8] C.F. Bornstein, J.L. Szwarcfiter. On clique convergent graphs. Graphs Combin. 11 (1995) 213-220. 
[9] C.F. Bornstein, J.L. Szwarcfiter. Iterated clique graphs with increasing diameters. J. Graph Theory 28 (1998) 147-154.

[10] B.L. Chen, K.W. Lih. Diameters of iterated clique graphs of chordal graphs. J. Graph Theory 14 (1990) 391-396.

[11] G.L. Chia. On self-clique graphs with given clique sizes. Discrete Math. 212 (2000) 185-189. Combinatorics and applications (Tianjin, 1996).

[12] S.A. Cook. The complexity of theorem-proving procedures. In Proc. 3rd Ann. ACM Symp. on Theory of Computing, pages 151-158, Association for Computing Machinery, New York, 1971.

[13] C.L. Deng, C.K. Lim. A class of clique-closed graphs. Discrete Math. 127 (1994) 131-137. Graph theory and applications (Hakone, 1990).

[14] R.D. Dutton, R.C. Brigham. On the radius and diameter of the clique graph. Discrete Math. 147 (1995) 293-295.

[15] F. Escalante. Über iterierte Clique-Graphen. Abh. Math. Sem. Univ. Hamburg 39 (1973) 59-68.

[16] M.E. Frías, V. Neumann-Lara, M.A. Pizaña. Dismantlings and iterated clique graphs. Submitted.

[17] M.E. Frías. Gráficas Iteradas de Clanes. PhD thesis, Instituto de Matemáticas, U.N.A.M., 2000.

[18] P. Galinier, M. Habib, C. Paul. Chordal graphs and their clique graphs. In Graph-theoretic concepts in computer science (Aachen, 1995), pages 358-371. Springer, Berlin, 1995.

[19] The GAP Group. GAP - Groups, Algorithms, and Programming, Version 4.3, 2002. (http://www.gap-system.org).

[20] M.R. Garey, D.S. Johnson. Computers and intractability. W. H. Freeman and Co., San Francisco, Calif., 1979. A guide to the theory of NP-completeness, A Series of Books in the Mathematical Sciences.

[21] M. Gutierrez, J. Meidanis. Algebraic theory for the clique operator. In preparation. 
[22] M. Gutierrez, J. Meidanis. The images under the clique operator of all graphs and of clique graphs. Submitted to Discrete Math.

[23] M. Gutierrez, J. Meidanis. On clique graph recognition. Submitted to Ars Combinatoria.

[24] M. Gutierrez, J. Meidanis. Recognizing clique graphs of directed edge path graphs. Submitted to Discrete Applied Mathematics.

[25] M. Gutierrez, J. Meidanis. The clique operator, set families, and their properties (extended abstract. In Electronic Notes on Discrete Mathematics, volume 7, pages 101-104. Proceedings of the Brazilian Symposium on Graphs, Algorithms and Combinatorics (Fortaleza, Ceará, Brazil. March 2001), 2001.

[26] T. Hamada, Y. Shimogaki. On the $n$-th power of a tree and its clique graph. Proc. Fac. Sci. Tokai Univ. 12 (1977) 11-14.

[27] R.C. Hamelink. A partial characterization of clique graphs. J. Combinatorial Theory 5 (1968) 192-197.

[28] F. Harary. Graph theory. Addison-Wesley Publishing Co., Reading, Mass.Menlo Park, Calif.-London, 1969.

[29] N. Hartsfield, G. Ringel. Clean triangulations. Combinatorica 11 (1991) 145155.

[30] S. Hazan, V. Neumann-Lara. Fixed points of posets and clique graphs. Order 13 (1996) 219-225.

[31] S.T. Hedetniemi, P.J. Slater. Line graphs of triangleless graphs and iterated clique graphs. In Graph theory and applications (Proc. Conf., Western Michigan Univ., Kalamazoo, Mich., 1972; dedicated to the memory of J. W. T. Youngs), pages 139-147. Lecture Notes in Math., Vol. 303. Springer, Berlin, 1972 .

[32] B. Hedman. Open questions in clique iteration. Graph Theory Newsletter (Western Michigan University) 12 (1983) .

[33] B. Hedman. Clique graphs of time graphs. J. Combin. Theory Ser. B 37 (1984) $270-278$. 
[34] B. Hedman. The maximum number of cliques in dense graphs. Discrete Math. 54 (1985) 161-166.

[35] B. Hedman. Diameters of iterated clique graphs. Hadronic J. 9 (1986) 273-276.

[36] P.J. Hilton, S. Wylie. Homology theory: An introduction to algebraic topology. Cambridge University Press, New York, 1960.

[37] F. Larrión, C.P. de Mello, A. Morgana, V. Neumann-Lara, M.A. Pizaña. The clique operator on cographs and serial graphs. Submitted.

[38] F. Larrión, V. Neumann-Lara. A family of clique divergent graphs with linear growth. Graphs Combin. 13 (1997) 263-266.

[39] F. Larrión, V. Neumann-Lara. Clique divergent graphs with unbounded sequence of diameters. Discrete Math. 197/198 (1999) 491-501. 16th British Combinatorial Conference (London, 1997).

[40] F. Larrión, V. Neumann-Lara. Locally $C_{6}$ graphs are clique divergent. Discrete Math. 215 (2000) 159-170.

[41] F. Larrión, V. Neumann-Lara. On clique-divergent graphs with linear growth. Discrete Math. 245 (2002) 139-153.

[42] F. Larrión, V. Neumann-Lara, M.A. Pizaña. Clique convergent surface triangulations. To appear in Matemática Contemporânea.

[43] F. Larrión, V. Neumann-Lara, M.A. Pizaña. Clique divergent clockwork graphs and partial orders. Submitted.

[44] F. Larrión, V. Neumann-Lara, M.A. Pizaña. On the homotopy type of clique graphs. To apper in Journal of the Brazilian Computer Society.

[45] F. Larrión, V. Neumann-Lara, M.A. Pizaña. Whitney triangulations, local girth and iterated clique graphs. To appear in Discrete Math.

[46] F. Larrión, V. Neumann-Lara, M.A. Pizaña. Clique divergent clockwork graphs and partial orders (extended abstract). In Electronic Notes on Discrete Mathematics, volume 7, pages 143-146. Proceedings of the Brazilian Symposium on Graphs, Algorithms and Combinatorics (Fortaleza, Ceará, Brazil. March 2001), 2001. 
[47] F. Larrión, V. Neumann-Lara, M.A. Pizaña, T.D. Porter. A hierarchy of selfclique graphs. Submitted.

[48] F. Larrión, V. Neumann-Lara, M.A. Pizaña, T.D. Porter. Recognizing selfclique graphs. Submitted.

[49] F. Larrión, V. Neumann-Lara, M.A. Pizaña, T.D. Porter. Self clique graphs with prescribed clique-sizes. Submitted.

[50] C.K. Lim, Y.H. Peng. On graphs without multicliqual edges. J. Graph Theory 5 (1981) 443-451.

[51] C.L. Lucchesi, C.P. de Mello, J.L. Szwarcfiter. On clique-complete graphs. Discrete Math. 183 (1998) 247-254.

[52] S. Mac Lane. Categories for the working mathematician. Springer-Verlag, New York, second edition, 1998.

[53] J.W. Moon, L. Moser. On cliques in graphs. Israel J. Math. 3 (1965) 23-28.

[54] V. Neumann-Lara. Personal communication.

[55] V. Neumann-Lara. A theory of expansive graphs. Preprint.

[56] V. Neumann-Lara. On clique-divergent graphs. Problèmes Combinatoires et Théorie des Graphes. Paris 260 (1978) 313-315.

[57] V. Neumann-Lara. Clique divergence in graphs. In Algebraic methods in graph theory, Vol. I, II (Szeged, 1978), pages 563-569. North-Holland, Amsterdam, 1981.

[58] V. Neumann-Lara. Clique divergence in graphs. some variations. Pub. Prelim. Inst. Mat. U.N.A.M, México 224 (1991) 1-14.

[59] C. Peyrat, D.F. Rall, P.J. Slater. On iterated clique graphs with increasing diameters. J. Graph Theory 10 (1986) 167-171.

[60] M.A. Pizaña. Distances and diameters on iterated clique graphs. Submitted.

[61] M.A. Pizaña. The icosahedron is clique divergent. To appear in Discrete Math. 
[62] M.A. Pizaña. Distances and diameters on iterated clique graphs (extended abstract). In Electronic Notes on Discrete Mathematics, volume 7, pages 181-184. Proceedings of the Brazilian Symposium on Graphs, Algorithms and Combinatorics (Fortaleza, Ceará, Brazil. March 2001), 2001.

[63] E. Prisner. Homology of the line graph and of related graph-valued functions. Arch. Math. 56 (1991) 400-404.

[64] E. Prisner. Convergence of iterated clique graphs. Discrete Math. 103 (1992) $199-207$.

[65] E. Prisner. Hereditary clique-Helly graphs. J. Combin. Math. Combin. Comput. 14 (1993) 216-220.

[66] E. Prisner. Graph dynamics. Longman, Harlow, 1995.

[67] E. Prisner. Graphs with few cliques. In Graph theory, combinatorics, and algorithms, Vol. 1, 2 (Kalamazoo, MI, 1992), pages 945-956. Wiley, New York, 1995.

[68] E. Prisner, J.L. Szwarcfiter. Recognizing clique graphs of directed and rooted path graphs. In Proceedings of the Third International Conference on Graphs and Optimization, GO-III (Leukerbad, 1998), volume 94(1-3), pages 321-328, 1999.

[69] F. Protti, J.L. Szwarcfiter. Clique-inverse graphs of $K_{3}$-free and $K_{4}$-free graphs. J. Graph Theory 35 (2000) 257-272.

[70] F. Protti, J.L. Szwarcfiter. On clique graphs with linear size. In Proceedings of the Thirty-first Southeastern International Conference on Combinatorics, Graph Theory and Computing (Boca Raton, FL, 2000), volume 143, pages 207-219, 2000.

[71] F.S. Roberts, J.H. Spencer. A characterization of clique graphs. J. Combinatorial Theory Ser. B 10 (1971) 102-108.

[72] J.J. Rotman. An introduction to the theory of groups. Springer-Verlag, New York, fourth edition, 1995.

[73] E.H. Spanier. Algebraic topology. Springer-Verlag, New York, 1981. Corrected reprint. 
[74] J.L. Szwarcfiter. A survey on clique graphs. In Recent Advances in Algorithms and Combinatorics. C. Linhares and B. Reed, eds., Springer-Verlag. To appear.

[75] J.L. Szwarcfiter. Recognizing clique-Helly graphs. Ars Combin. 45 (1997) 2932.

[76] J.L. Szwarcfiter. Personal communication.

[77] W.T. Tutte. A census of plane triangulations. Canad. J. Math. 14 (1962) $21-28$.

[78] W.D. Wallis, J.L. Wu. Squares, clique graphs and chordality. J. Graph Theory 20 (1995) 37-45.

[79] H. Whitney. A theorem on graphs. Ann. Math. 32 (1931) 378-390. 


\section{Índice}

A

admisible, 53

adyacentes, 4

automorfa, 53

\section{B}

Bornstein, v, 8, 13, 18, 24, 27, 28, 87

Brigham, v, 15-18, 24

\section{C}

cíclicamente segmentada, 34

centro

de una corbata, 77

de una estrella, 77

cimiento, 18

clan, 1

de clanes, 8

iterado, 3

clan convergente, 2

clan divergente, 2

clan-Helly, 7

clase $k$-cerrada, 7

coafín, 54

complejo, 5

gráfica subyacente a un, 5

simplicial, 5

completo, 54 conjetura

de la esfera, 82, 90

del disco, 82, 90

conjunto de distancias, 4

cono, 7

corbata, 9

centro de una, 77

exótica, 77

pura, 29

corona de un reloj, 47

cubrimiento triangular, 12

número de hojas de un, 12

cuello, 71

cuello local, 71

\section{D}

defecto de Helly, 8

diámetro, 4

distancia unidireccional, 63

divergente en sumandos, 61

dodecaedro estelado, 66

$k$-carácter del, 66

dominación

entre gráficas, 56

entre vértices, 4

Dutton, v, 15-18, 24 
E

Escalante, v, 2, 7-9, 78

estrella, 9

centro de una, 77

morfismo, 9

extensión, 20

\section{F}

fórmula de la distancia, 18

Frías, v, 11, 49, 89

\section{G}

grado de un vértice, 5

gráfica(s)

automorfa, 53

cíclicamente segmentada, 34

clan convergente, 2

clan divergente, 2

primitiva, 10

clan-Helly, 7

coafín, 54

completa, 5

de clanes, 1

de completas, 3

de Johnson, 25

de relojería, 33

diámetro de una, 4

divergente en sumandos, 61

dominación entre, 56

iteradas de clanes, 1

$k$-carácter de una, 2

$k$-convergente, 2

$k$-divergente, 2

$k$-nula, 2

$k$-periódica, 2

$k^{n}$-invariante, 2

localmente $\mathcal{H}, 5$ localmente $H, 5$

localmente cíclica, 5

morfismo de, 4

orden de una, 3

punteada, 5

$r$-coafín

rango de una, 59

$r$-coafín, 54

radio de una, 4

subyacente

a un complejo simplicial, 5

a una triangulación, 5

Gutierrez, v

\section{$\mathbf{H}$}

Hedetniemi, vi, 1, 2

Hedman, v, vi, 7, 15, 24, 31

Helly

propiedad de, 7

hojas

de un cubrimiento triangular, 12

de un isomorfismo local, 12

\section{I}

icosaedro, 65

$k$-carácter del, 65

sucesión de órdenes del, 10

índice

de transición, 2

isomorfismo local, 12

número de hojas de un, 12

K

$k$-carácter, 2

$k$-cerrada, 7

$k$-convergente, 2

$k$-divergente, 2 
$k$-nula, 2

$k$-periódica, 2

$k^{n}$-invariante, 2

\section{L}

Larrión, v, vi, 6, 8, 12, 24, 33

localmente $\mathcal{H}, 5$

localmente $H, 5$

localmente cíclica, 5

\section{M}

morfismo, 4

admisible, 53

completo, 54

estrella, 9

extensión de un, 20

\section{$\mathbf{N}$}

Neumann-Lara, v, vi, 2-4, 6, 8, 11, 12, $24,33,49,53,56-59,63,65,89$

núcleo de un reloj, 47

número de hojas

de un cubrimiento triangular, 12

de un isomorfismo local, 12

\section{$\mathrm{O}$}

octaedro, 5

$k$-carácter del, 2

$n$-dimensional, 5

operación

tac, 42

tic, 42

orden de una gráfica, 3

\section{$\mathbf{P}$}

periodo, 2

Peyrat, vi, 13, 16, 24

Prisner, vi, 2, 11 problema

de la computabilidad

de la $k$-nulidad, 45,89

del $k$-carácter, 45,89

del diámetro, 28, 89

producto fuerte, 4

de gráficas $r$-coafines, 54

de gráficas automorfas, 54

propiedad de Helly, 7

\section{R}

$r$-coafín, 54

radio, 4

radio local, 4

Rall, vi, 13, 16, 24

rango, 59

reloj, 34

antiguo, 47

corona de un, 47

núcleo de un, 47

$r$-coafín, 63

segmentos de un, 34

vértices nuevos de un, 35

vértices viejos de un, 35

retracción, 11

fuerte, 11

retracto, 11

retracto fuerte, 11

S

sección, 11

segmentación cíclica, 34

segmento(s), 34

anterior, 34

bueno, 35

de un reloj, 34

malo, 35 
siguiente, 34

simplejo, 5

Slater, vi, 1, 2, 13, 16, 24

suma r-coafín, 59

suma de Zykov, 59

suma punteada, 5

suma segmentada, 46

superficie, 5

cerrada, 6

compacta, 6

Szwarcfiter, vi, 7, 8, 13, 18, 24, 27, 28, 87

$\mathrm{T}$

tac, 42

teorema

de Escalante, 8

de la divergencia en sumandos, 61

de la retracción, 11

fuerte, 11

de Szwarcfiter, 7

del cubrimiento triangular, 12

del cuello local grande, 77

del diámetro

de Bornstein y Szwarcfiter, 24

tic, 42

triangulación, 5

de Whitney, 5

gráfica subyacente a una, 5

limpia, 6

triángulo extendido, 7

U

1-esqueleto, 5

\section{V}

vecindad

cerrada de un vértice, 4 de un conjunto, 4

de un vértice, 3

vecinos

de un vértice, 4

vértice(s)

adyacentes, 4

cuello local en un, 71

dominación entre, 4

dominado, 4

grado de un, 5

nuevos, 35

radio local en un, 4

vecindad cerrada de un, 4

vecindad de un, 3

vecinos de un, 4

viejos, 35

W

Whitney

triangulación de, 5

\section{$\mathbf{Z}$}

Zykov

suma de, 59 